\title{
Power corrections to TMD factorization for Z-boson production
}

\author{
I. Balitsky ${ }^{a, b}$ and A. Tarasov ${ }^{c}$ \\ ${ }^{a}$ Physics Department, Old Dominion University, \\ Norfolk, VA 23529, U.S.A. \\ ${ }^{b}$ Thomas Jefferson National Accelerator Facility, \\ Newport News, VA 23606, U.S.A. \\ ${ }^{c}$ Physics Department, Brookhaven National Laboratory, \\ Upton, NY 11973, U.S.A. \\ E-mail: balitsky@jlab.org, atarasov@bnl.gov
}

ABSTRACT: A typical factorization formula for production of a particle with a small transverse momentum in hadron-hadron collisions is given by a convolution of two TMD parton densities with cross section of production of the final particle by the two partons. For practical applications at a given transverse momentum, though, one should estimate at what momenta the power corrections to the TMD factorization formula become essential. In this paper we calculate the first power corrections to TMD factorization formula for Z-boson production and Drell-Yan process in high-energy hadron-hadron collisions. At the leading order in $N_{c}$ power corrections are expressed in terms of leading power TMDs by $\mathrm{QCD}$ equations of motion.

KEYWORDS: NLO Computations, QCD Phenomenology

ArXIV EPRINT: 1712.09389 


\section{Contents}

1 Introduction 1

$\begin{array}{lll}2 & \text { TMD factorization from functional integral } & 2\end{array}$

3 Power corrections and solution of classical YM equations $\quad 6$

$\begin{array}{lll}3.1 & \text { Power counting for background fields } & 6\end{array}$

$\begin{array}{lll}3.2 & \text { Approximate solution of classical equations } & 7\end{array}$

$\begin{array}{lll}3.3 & \text { Power expansion of classical quark fields } & 11\end{array}$

4 Leading power corrections at $s \gg Q^{2} \gg q_{\perp}^{2} \quad 12$

4.1 Leading contribution and power corrections from $\mathcal{J}_{A B}^{\mu}(x) \mathcal{J}_{B A \mu}(0)$ terms 13

$\begin{array}{lll}4.2 & \text { Leading power contribution } & 14\end{array}$

$\begin{array}{ll}\text { 4.2.1 Parametrization of leading matrix elements } & 15\end{array}$

$\begin{array}{lll}4.3 & \text { Power corrections from } \mathcal{J}_{A B}^{\mu}(x) \mathcal{J}_{B A \mu}(0) \text { terms } & 16\end{array}$

4.3.1 Fifth line in eq. (4.19): the leading term in $\frac{1}{N_{c}} \quad 17$

4.3.2 Parametrization of matrix elements from section 4.3.1 20

$\begin{array}{lll}\text { 4.3.3 Sixth line in eq. (4.19) } & 21\end{array}$

4.3.4 Parametrization of matrix elements from section 4.3.3 24

4.4 Power corrections from $\mathcal{J}_{A}^{\mu}(x) \mathcal{J}_{B \mu}(0)$ terms 24

4.4.1 Last two lines in eq. (4.56) 25

$\begin{array}{lll}\text { 4.4.2 Parametrization of TMDs from section 4.4.1 } & 27\end{array}$

5 Results and estimates $\quad 28$

6 Power corrections for Drell-Yan process $\quad 30$

$\begin{array}{lll}7 & \text { Conclusions and outlook } & 31\end{array}$

$\begin{array}{ll}\text { A Next-to-leading quark fields } & 33\end{array}$

B Formulas with Dirac matrices $\quad 34$

C Subleading power corrections $\quad 35$

C.1 Second, third, and fourth lines in eq. (4.19) 35

$\begin{array}{lll}\text { C.2 Second to fifth lines in eq. (4.56) } & 37\end{array}$

$\begin{array}{lll}\text { C.3 Gluon power corrections from } \mathcal{J}_{A}^{\mu}(x) \mathcal{J}_{A \mu}(0) \text { terms } & 38\end{array}$

$\begin{array}{lll}\text { C.4 Power corrections from } \Psi^{(1)} \text { fields } & 39\end{array}$ 


\section{Introduction}

A typical analysis of differential cross section of particle production in hadron-hadron collisions at small momentum transfer of the produced particle is performed with the help of TMD factorization [1-10]. However, the question of how small should be the momentum transfer in order for leading power TMD analysis to be successful cannot be resolved at the leading-power level. The sketch of the factorization formula for the differential cross section is $[1,11]$

$$
\begin{aligned}
\frac{d \sigma}{d \eta d^{2} q_{\perp}}= & \sum_{f} \int d^{2} b_{\perp} e^{i(q, b)} \perp_{f / A}\left(x_{A}, b_{\perp}, \eta\right) \mathcal{D}_{f / B}\left(x_{B}, b_{\perp}, \eta\right) \sigma(f f \rightarrow H) \\
& + \text { power corrections }+\mathrm{Y}-\text { terms }
\end{aligned}
$$

where $\eta$ is the rapidity, $q$ is the momentum of the produced particle in the hadron frame (see ref. [1]), $\mathcal{D}_{f / A}\left(x, z_{\perp}, \eta\right)$ is the TMD density of a parton $f$ in hadron $A$, and $\sigma(f f \rightarrow H)$ is the cross section of production of particle $H$ in the scattering of two partons. The common wisdom is that when we increase transverse momentum $q_{\perp}^{2}$ of the produced hadron, at first the leading power TMD analysis with (nonperturbative) TMDs applies, then at some point power corrections kick in, and finally at $q_{\perp}^{2} \sim Q^{2}$, where $Q^{2}=q^{2}$, they are transformed into so-called Y-term making smooth transition to collinear factorization formulas. In this paper we try to answer the question about the first transition, namely at what $q_{\perp}^{2}$ power corrections become significant.

In our recent paper [12] we calculated power corrections $\sim \frac{q_{\perp}^{2}}{Q^{2}}$ for Higgs boson production by gluon-gluon fusion. The result was a TMD factorization formula with matrix elements of three-gluon operators divided by an extra power of $m_{H}^{2}$. In this paper we calculate power corrections $\sim \frac{q_{\perp}^{2}}{Q^{2}}$ for Z-boson production which are determined by quarkquark-gluon operators. In the leading order Z-boson production was studied in [13-21]. The interesting (and unexpected) result of our paper is that at the leading- $N_{c}$ level matrix elements of the relevant quark-quark-gluon operators can be expressed in terms of leading power quark-antiquark TMDs by QCD equations of motion (see ref. [22]). The method of calculation is very similar to that of ref. [12] so we will streamline the discussion of the general approach and pay attention to details specific to quark operators.

The paper is organized as follows. In section 2 we derive the TMD factorization from the double functional integral for the cross section of particle production. In section 3 , which is central to our approach, we explain the method of calculation of power corrections based on a solution of classical Yang-Mills equations. In section 4 we find the leading power correction to particle production in the region $s \gg Q^{2} \gg q_{\perp}^{2}$. In section 5 we perform the order-of-magnitude estimate of power corrections and in section 6 present our result for power corrections to the Drell-Yan cross section. The necessary technical details and discussion of subleading power corrections can be found in appendix. 


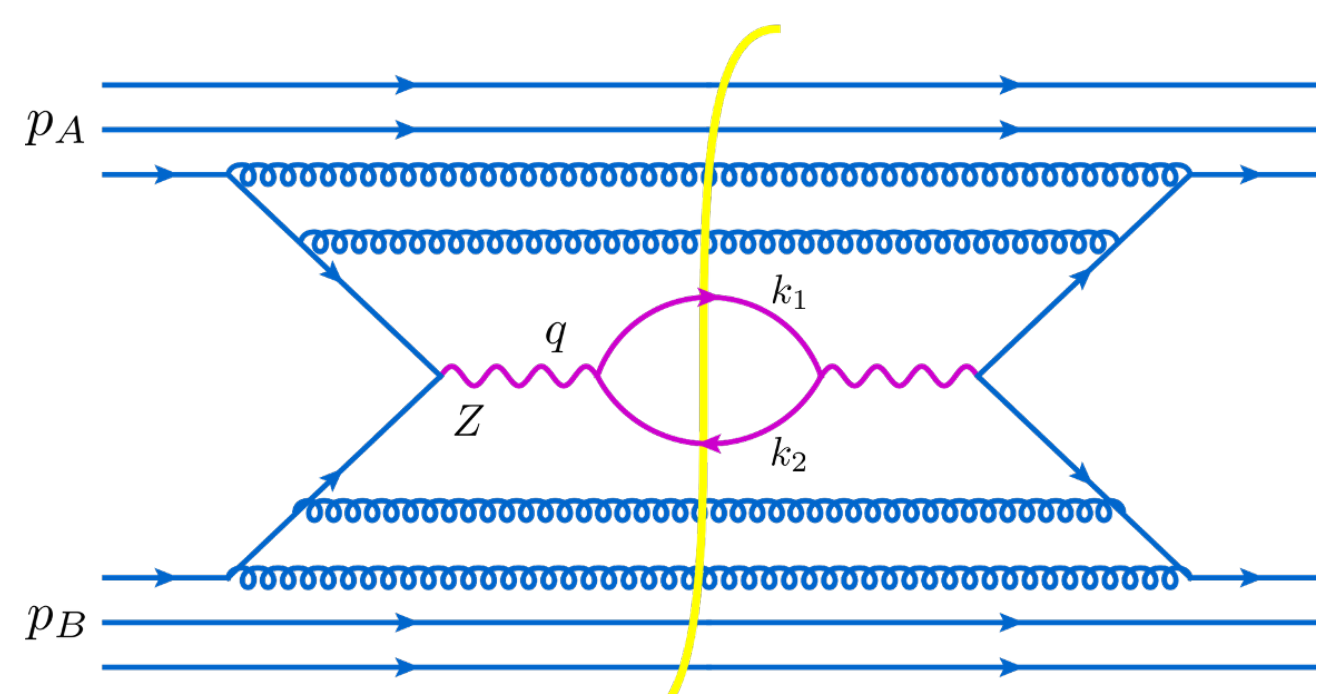

Figure 1. Z-boson production in hadron-hadron collisions.

\section{TMD factorization from functional integral}

We consider Z-boson production in the Drell-Yan reaction illustrated in figure 1:

$$
h_{A}\left(p_{A}\right)+h_{B}\left(p_{A}\right) \rightarrow Z(q)+X \rightarrow l_{1}\left(k_{1}\right)+l_{2}\left(k_{2}\right)+X,
$$

where $h_{A, B}$ denote the colliding hadrons, and $l_{1,2}$ the outgoing lepton pair with total momentum $q=k_{1}+k_{2}$.

The relevant term of the Lagrangian for the fermion fields $\psi_{i}$ describing coupling between fermions and Z-boson is $\left(s_{W} \equiv \sin \theta_{W}, c_{W} \equiv \cos \theta_{W}\right)$

$$
\mathcal{L}_{Z}=\int d^{4} x J_{\mu} Z^{\mu}(x), \quad J_{\mu}=-\frac{e}{2 s_{W} c_{W}} \sum_{i} \bar{\psi}_{i} \gamma_{\mu}\left(g_{i}^{V}-g_{i}^{A} \gamma_{5}\right) \psi_{i},
$$

where sum goes over different types of fermions, and coupling constants $g_{i}^{V}=\left(t_{3}^{L}\right)_{i}-2 q_{i} s_{W}^{2}$ and $g_{i}^{A}=\left(t_{3}^{L}\right)_{i}$ are defined by week isospin $\left(t_{3}^{L}\right)_{i}$ of the fermion $i$, see ref. [23]. In this paper we take into account only $u, d, s, c$ quarks and $e, \mu$ leptons. We consider all fermions to be massless.

The differential cross section of Z-boson production with subsequent decay into $e^{+} e^{-}$ (or $\mu^{+} \mu^{-}$) pair is

$$
\frac{d \sigma}{d Q^{2} d y d q_{\perp}^{2}}=\frac{e^{2} Q^{2}}{192 s s_{W}^{2} c_{W}^{2}} \frac{1-4 s_{W}^{2}+8 s_{W}^{4}}{\left(m_{Z}^{2}-Q^{2}\right)^{2}+\Gamma_{Z}^{2} m_{Z}^{2}}\left[-W\left(p_{A}, p_{B}, q\right)\right]
$$

where we defined the "hadronic tensor" $W\left(p_{A}, p_{B}, q\right)$ as

$$
\begin{aligned}
W\left(p_{A}, p_{B}, q\right) & \stackrel{\text { def }}{=} \frac{1}{(2 \pi)^{4}} \sum_{X} \int d^{4} x e^{-i q x}\left\langle p_{A}, p_{B}\left|J_{\mu}(x)\right| X\right\rangle\left\langle X\left|J^{\mu}(0)\right| p_{A}, p_{B}\right\rangle \\
& =\frac{1}{(2 \pi)^{4}} \int d^{4} x e^{-i q x}\left\langle p_{A}, p_{B}\left|J_{\mu}(x) J^{\mu}(0)\right| p_{A}, p_{B}\right\rangle .
\end{aligned}
$$


As usual, $\sum_{X}$ denotes the sum over full set of "out" states. It should be mentioned that there is a power correction coming from the leptonic tensor term $\sim q^{\mu} q^{\nu}$. However, if we consider quarks to be massless, the only effect of the $q^{\mu} q^{\nu}$ term comes from the (square of) axial anomaly which has an extra factor $\alpha_{s}^{2}$, and such two-loop factor is beyond our tree approximation.

The sum over full set of "out" states in eq. (2.4) can be represented by a double functional integral

$$
\begin{aligned}
& (2 \pi)^{4} W\left(p_{A}, p_{B}, q\right)=\sum_{X} \int d^{4} x e^{-i q x}\left\langle p_{A}, p_{B}\left|J^{\mu}(x)\right| X\right\rangle\left\langle X\left|J_{\mu}(0)\right| p_{A}, p_{B}\right\rangle \\
& =\lim _{t_{i} \rightarrow-\infty}^{t_{f} \rightarrow \infty} \int d^{4} x e^{-i q x} \int^{\tilde{A}\left(t_{f}\right)=A\left(t_{f}\right)} D \tilde{A} \tilde{A}_{\mu} D A_{\mu} \int^{\tilde{\psi}\left(t_{f}\right)=\psi\left(t_{f}\right)} D \tilde{\bar{\psi}} D \tilde{\psi} D \bar{\psi} D \psi \Psi_{p_{A}}^{*}\left(\overrightarrow{\tilde{A}}\left(t_{i}\right), \tilde{\psi}\left(t_{i}\right)\right) \\
& \quad \times \Psi_{p_{B}}^{*}\left(\overrightarrow{\tilde{A}}\left(t_{i}\right), \tilde{\psi}\left(t_{i}\right)\right) e^{-i S_{\mathrm{QCD}}(\tilde{A}, \tilde{\psi})} e^{i S_{\mathrm{QCD}}(A, \psi)} \tilde{J}_{\mu}(x) J^{\mu}(0) \Psi_{p_{A}}\left(\vec{A}\left(t_{i}\right), \psi\left(t_{i}\right)\right) \Psi_{p_{B}}\left(\vec{A}\left(t_{i}\right), \psi\left(t_{i}\right)\right) .
\end{aligned}
$$

In this double functional integral the amplitude $\left\langle X\left|J_{\mu}(0)\right| p_{A}, p_{B}\right\rangle$ is given by the integral over $\psi, A$ fields whereas the complex conjugate amplitude $\left\langle p_{A}, p_{B}\left|J^{\mu}(x)\right| X\right\rangle$ is represented by the integral over $\tilde{\psi}, \tilde{A}$ fields. Also, $\Psi_{p}\left(\vec{A}\left(t_{i}\right), \psi\left(t_{i}\right)\right)$ denotes the proton wave function at the initial time $t_{i}$ and the boundary conditions $\tilde{A}\left(t_{f}\right)=A\left(t_{f}\right)$ and $\tilde{\psi}\left(t_{f}\right)=\psi\left(t_{f}\right)$ reflect the sum over all states $X$, cf. refs. [24-26].

We use Sudakov variables $p=\alpha p_{1}+\beta p_{2}+p_{\perp}$, where $p_{1}$ and $p_{2}$ are light-like vectors close to $p_{A}$ and $p_{B}$, and the notations $x_{\bullet} \equiv x_{\mu} p_{1}^{\mu}$ and $x_{*} \equiv x_{\mu} p_{2}^{\mu}$ for the dimensionless light-cone coordinates $\left(x_{*}=\sqrt{\frac{s}{2}} x_{+}\right.$and $\left.x_{\bullet}=\sqrt{\frac{s}{2}} x_{-}\right)$. Our metric is $g^{\mu \nu}=(1,-1,-1,-1)$ so that $p \cdot q=\left(\alpha_{p} \beta_{q}+\alpha_{q} \beta_{p}\right) \frac{s}{2}-(p, q)_{\perp}$ where $(p, q)_{\perp} \equiv-p_{i} q^{i}$. Throughout the paper, the sum over the Latin indices $i, j, \ldots$ runs over two transverse components while the sum over Greek indices $\mu, \nu, \ldots$ runs over four components as usual.

Following ref. [12] we separate quark and gluon fields in the functional integral (2.5) into three sectors (see figure 2): "projectile" fields $A_{\mu}, \psi_{A}$ with $|\beta|<\sigma_{a}$, "target" fields $B_{\mu}, \psi_{B}$ with $|\alpha|<\sigma_{b}$ and "central rapidity" fields $C_{\mu}, \psi_{C}$ with $|\alpha|>\sigma_{b}$ and $|\beta|>\sigma_{a}$ and get

$$
\begin{aligned}
& W\left(p_{A}, p_{B}, q\right)=\frac{1}{(2 \pi)^{4}} \int d^{4} x e^{-i q x} \int^{\tilde{A}\left(t_{f}\right)=A\left(t_{f}\right)} D \tilde{A}_{\mu} D A_{\mu} \int^{\tilde{\psi}_{A}\left(t_{f}\right)=\psi_{A}\left(t_{f}\right)} D \bar{\psi}_{A} D \psi_{A} \\
& \times D \tilde{\bar{\psi}}_{A} D \tilde{\psi}_{A} e^{-i S_{\mathrm{QCD}}\left(\tilde{A}, \tilde{\psi}_{A}\right)} e^{i S_{\mathrm{QCD}}\left(A, \psi_{A}\right)} \Psi_{p_{A}}^{*}\left(\overrightarrow{\tilde{A}}\left(t_{i}\right), \tilde{\psi}_{A}\left(t_{i}\right)\right) \Psi_{p_{A}}\left(\vec{A}\left(t_{i}\right), \psi_{A}\left(t_{i}\right)\right) \\
& \times \int^{\tilde{B}\left(t_{f}\right)=B\left(t_{f}\right)} D \tilde{B}_{\mu} D B_{\mu} \int^{\tilde{\psi}_{B}\left(t_{f}\right)=\psi_{B}\left(t_{f}\right)} D \bar{\psi}_{B} D \psi_{B} D \tilde{\bar{\psi}}_{B} D \tilde{\psi}_{B} \\
& \times e^{-i S_{\mathrm{QCD}}\left(\tilde{B}, \tilde{\psi}_{B}\right)} e^{i S_{\mathrm{QCD}}\left(B, \psi_{B}\right)} \Psi_{p_{B}}^{*}\left(\overrightarrow{\tilde{B}}\left(t_{i}\right), \tilde{\psi}_{B}\left(t_{i}\right)\right) \Psi_{p_{B}}\left(\vec{B}\left(t_{i}\right), \psi_{B}\left(t_{i}\right)\right) \\
& \times \int D C_{\mu} \int^{\tilde{C}\left(t_{f}\right)=C\left(t_{f}\right)} D \tilde{C}_{\mu} \int D \bar{\psi}_{C} D \psi_{C} \int_{\tilde{\psi}_{C}\left(t_{f}\right)=\psi_{C}\left(t_{f}\right)}^{D \tilde{\psi}_{C} D \tilde{\psi}_{C}} \tilde{J}_{\mu}(x) J^{\mu}(0) e^{-i \tilde{S}_{C}+i S_{C},},
\end{aligned}
$$

where $S_{C}=S_{\mathrm{QCD}}\left(C+A+B, \psi_{C}+\psi_{A}+\psi_{B}\right)-S_{\mathrm{QCD}}\left(A, \psi_{A}\right)-S_{\mathrm{QCD}}\left(B, \psi_{B}\right)$ and similarly for $\tilde{S}_{C \cdot}{ }^{1}$

\footnotetext{
${ }^{1}$ This procedure is obviously gauge-dependent. We have in mind factorization in covariant-type gauge, e.g. Feynman gauge.
} 


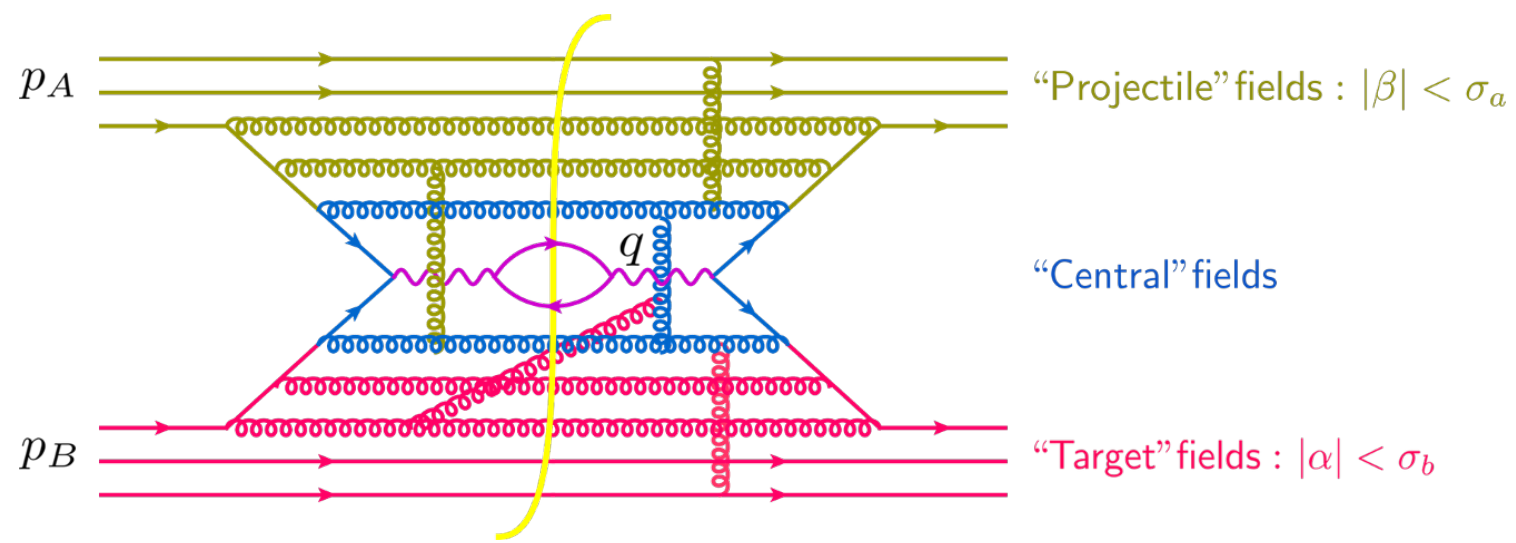

Figure 2. Rapidity factorization for particle production.

Our goal is to integrate over central fields and get the amplitude in the factorized form, i.e. as a product of functional integrals over $A$ fields representing projectile matrix elements (TMDs of the projectile) and functional integrals over $B$ fields representing target matrix elements (TMDs of the target).

In the spirit of background-field method, we "freeze" projectile and target fields and get a sum of diagrams in these external fields. Since $|\beta|<\sigma_{a}$ in the projectile fields and $|\alpha|<\sigma_{b}$ in the target fields, at the tree-level one can set with power accuracy $\beta=0$ for the projectile fields and $\alpha=0$ for the target fields - the corrections will be $O\left(\frac{m_{N}^{2}}{\sigma_{a} s}\right)$ and $O\left(\frac{m_{N}^{2}}{\sigma_{b} s}\right)$, where $m_{N}$ is the hadron's mass. Beyond the tree level, one should expect that the integration over $C$ fields will produce the logarithms of the cutoffs $\sigma_{a}$ and $\sigma_{b}$ which will cancel with the corresponding logs in gluon TMDs of the projectile and the target. The result of integration over C-fields has the schematic form

$$
\begin{gathered}
\int D C_{\mu} \int^{\tilde{C}\left(t_{f}\right)=C\left(t_{f}\right)} D \tilde{C}_{\mu} \int D \bar{\psi}_{C} D \psi_{C} \int_{\tilde{\psi}_{C}\left(t_{f}\right)=\psi_{C}\left(t_{f}\right)} D \tilde{\bar{\psi}}_{C} D \tilde{\psi}_{C} \tilde{J}_{\mu}(x) J^{\mu}(0) e^{-i \tilde{S}_{C}+i S_{C}} \\
=e^{S_{\mathrm{eff}}\left(A, \psi_{A}, \tilde{A}, \tilde{\psi}_{A} ; B, \psi_{B}, \tilde{B}, \tilde{\psi}_{B}\right)} \mathcal{O}\left(q, x ; A, \psi_{A}, \tilde{A}, \tilde{\psi}_{A} ; B, \psi_{B}, \tilde{B}, \tilde{\psi}_{B}\right),
\end{gathered}
$$

where $\mathcal{O}\left(q, x ; A, \psi_{A}, \tilde{A}, \tilde{\psi}_{A} ; B, \psi_{B}, \tilde{B}, \tilde{\psi}_{B}\right)$ is a sum of diagrams connected to $\tilde{J}_{\mu}(x) J^{\mu}(0)$ and $e^{S_{\text {eff }}}$ represents a sum of disconnected diagrams ("vacuum bubbles") in external fields. As usual, since the rapidities of central $C$ fields and of $A, B$ fields are very different, the result of integration over $C$ fields is expressed in terms of Wilson-line operators made form $A$ and $B$ fields.

After integration over $C$ fields the amplitude (2.5) can be rewritten as

$$
\begin{aligned}
& W\left(p_{A}, p_{B}, q\right)=\frac{1}{(2 \pi)^{4}} \int d^{4} x e^{-i q x} \int^{\tilde{A}\left(t_{f}\right)=A\left(t_{f}\right)} D \tilde{A}_{\mu} D A_{\mu} \int^{\tilde{\psi}_{A}\left(t_{f}\right)=\psi_{A}\left(t_{f}\right)} D \bar{\psi}_{A} D \psi_{A} D \tilde{\bar{\psi}}_{A} D \tilde{\psi}_{A} \\
& \times e^{-i S_{\mathrm{QCD}}\left(\tilde{A}, \tilde{\psi}_{A}\right)} e^{i S_{\mathrm{QCD}}\left(A, \psi_{A}\right)} \Psi_{p_{A}}^{*}\left(\overrightarrow{\tilde{A}}\left(t_{i}\right), \tilde{\psi}_{A}\left(t_{i}\right)\right) \Psi_{p_{A}}\left(\vec{A}\left(t_{i}\right), \psi_{A}\left(t_{i}\right)\right) \int^{\tilde{B}\left(t_{f}\right)=B\left(t_{f}\right)} D \tilde{B}_{\mu} D B_{\mu} \\
& \times \int^{\tilde{\psi}_{B}\left(t_{f}\right)=\psi_{B}\left(t_{f}\right)} D \bar{\psi}_{B} D \psi_{B} D \tilde{\bar{\psi}}_{B} D \tilde{\psi}_{B} e^{-i S_{\mathrm{QCD}}\left(\tilde{B}, \tilde{\psi}_{B}\right)} e^{i S_{\mathrm{QCD}}\left(B, \psi_{B}\right)} \Psi_{p_{B}}^{*}\left(\overrightarrow{\tilde{B}}\left(t_{i}\right), \tilde{\psi}_{B}\left(t_{i}\right)\right) \\
& \times \Psi_{p_{B}}\left(\vec{B}\left(t_{i}\right), \psi_{B}\left(t_{i}\right)\right) e^{S_{\mathrm{eff}}\left(A, \psi_{A}, \tilde{A}, \tilde{\psi}_{A} ; B, \psi_{B}, \tilde{B}, \tilde{\psi}_{B}\right)} \mathcal{O}\left(q, x ; A, \psi_{A}, \tilde{A}, \tilde{\psi}_{A} ; B, \psi_{B}, \tilde{B}, \tilde{\psi}_{B}\right) .
\end{aligned}
$$


From integrals over projectile and target fields in the above equation we see that the functional integral over $C$ fields should be done in the background of $A$ and $B$ fields satisfying

$$
\tilde{A}\left(t_{f}\right)=A\left(t_{f}\right), \quad \tilde{\psi}_{A}\left(t_{f}\right)=\psi_{A}\left(t_{f}\right) \text { and } \quad \tilde{B}\left(t_{f}\right)=B\left(t_{f}\right), \quad \tilde{\psi}_{B}\left(t_{f}\right)=\psi_{B}\left(t_{f}\right) .
$$

Combining this with our approximation that at the tree level $\beta=0$ for $A, \tilde{A}$ fields and $\alpha=0$ for $B, \tilde{B}$ fields, which corresponds to $A=A\left(x_{\bullet}, x_{\perp}\right), \tilde{A}=\tilde{A}\left(x_{\bullet}, x_{\perp}\right)$ and $B=B\left(x_{*}, x_{\perp}\right)$, $\tilde{B}=\tilde{B}\left(x_{*}, x_{\perp}\right)$, we see that for the purpose of calculation of the functional integral over central fields (2.7) we can set

$$
\text { and } \begin{array}{rlrl}
A\left(x_{\bullet}, x_{\perp}\right) & =\tilde{A}\left(x_{\bullet}, x_{\perp}\right), & \psi_{A}\left(x_{\bullet}, x_{\perp}\right) & =\tilde{\psi}_{A}\left(x_{\bullet}, x_{\perp}\right) \\
& B\left(x_{*}, x_{\perp}\right) & =\tilde{B}\left(x_{*}, x_{\perp}\right), \quad \psi_{B}\left(x_{*}, x_{\perp}\right)=\tilde{\psi}_{B}\left(x_{*}, x_{\perp}\right) .
\end{array}
$$

In other words, since $A, \psi$ and $\tilde{A}, \tilde{\psi}$ do not depend on $x_{*}$, if they coincide at $x_{*}=\infty$ they should coincide everywhere. Similarly, since $B, \psi_{B}$ and $\tilde{B}, \tilde{\psi}_{B}$ do not depend on $x_{\bullet}$, if they coincide at $x_{\bullet}=\infty$ they should be equal.

Next, in ref. [12] it was demonstrated that due to eqs. (2.10) the effective action $S_{\text {eff }}\left(A, \psi_{A}, \tilde{A}, \tilde{\psi}_{A} ; B, \psi_{B}, \tilde{B}, \tilde{\psi}_{B}\right)$ vanishes for background fields satisfying conditions (2.9). ${ }^{2}$

Summarizing, we see that at the tree level in our approximation

$$
\begin{aligned}
& \int D C_{\mu} \int^{\tilde{C}\left(t_{f}\right)=C\left(t_{f}\right)} D \tilde{C}_{\mu} \int D \bar{\psi}_{C} D \psi_{C} \int^{\tilde{\psi}_{C}\left(t_{f}\right)=\psi_{C}\left(t_{f}\right)} D \tilde{\bar{\psi}}_{C} D \tilde{\psi}_{C} \tilde{J}_{\mu}(x) J^{\mu}(0) e^{-i \tilde{S}_{C}+i S_{C}} \\
& \quad=\mathcal{O}\left(q, x ; A, \psi_{A} ; B, \psi_{B}\right)
\end{aligned}
$$

where now $S_{C}=S_{\mathrm{QCD}}\left(C+A+B, \psi_{C}+\psi_{A}+\psi_{B}\right)-S_{\mathrm{QCD}}\left(A, \psi_{A}\right)-S_{\mathrm{QCD}}\left(B, \psi_{B}\right)$ and $\tilde{S}_{C}=S_{\mathrm{QCD}}\left(\tilde{C}+A+B, \tilde{\psi}_{C}+\psi_{A}+\psi_{B}\right)-S_{\mathrm{QCD}}\left(A, \psi_{A}\right)-S_{\mathrm{QCD}}\left(B, \psi_{B}\right)$. It is well known that in the tree approximation the double functional integral (2.11) is given by a set of retarded Green functions in the background fields [27-29] (see also appendix A of ref. [12] for the proof). Since the double functional integral (2.11) is given by a set of retarded Green functions (in the background fields $A$ and $B$ ), calculation of the tree-level contribution to $\bar{\psi} \gamma_{\mu} \psi$ in the r.h.s. of eq. (2.11), is equivalent to solving YM equation for $\psi(x)$ (and $A_{\mu}(x)$ ) with boundary conditions such that the solution has the same asymptotics at $t \rightarrow-\infty$ as the superposition of incoming projectile and target background fields.

The hadronic tensor (2.8) can now be represented $\mathrm{as}^{3}$

$$
W\left(p_{A}, p_{B}, q\right)=\frac{1}{(2 \pi)^{4}} \int d^{4} x e^{-i q x}\left\langle p_{A}\left|\left\langle p_{B}\left|\hat{\mathcal{O}}\left(q, x ; \hat{A}, \hat{\psi}_{A} ; \hat{B}, \hat{\psi}_{B}\right)\right| p_{A}\right\rangle\right| p_{B}\right\rangle,
$$

\footnotetext{
${ }^{2}$ It corresponds to cancellation of so-called "Glauber gluons", see discussion in ref. [1].

${ }^{3}$ As discussed in ref. [12], there is a subtle point in the promotion of background fields to operators. When we calculate $\mathcal{O}$ as the r.h.s. of eq. (2.11) the fields $\Phi_{A}$ and $\Phi_{B}$ are c-numbers; on the other hand, after functional integration in eq. (2.5) they become operators which must be time-ordered in the right sector and anti-time-ordered in the left sector. Fortunately, as we shall see below, all these operators are separated either by space-like distances or light-cone distances so all of them (anti) commute and thus can be treated as $c$-numbers.
} 
where $\hat{\mathcal{O}}\left(q, x ; \hat{A}, \hat{\psi}_{A} ; \hat{B}, \hat{\psi}_{B}\right)$ should be expanded in a series in $\hat{A}, \hat{\psi}_{A}, \hat{B}, \hat{\psi}_{B}$ operators and evaluated between the corresponding (projectile or target) states: if

$$
\hat{\mathcal{O}}\left(q, x ; \hat{A}, \hat{\psi}_{A} ; \hat{B}, \hat{\psi}_{B}\right)=\sum_{m, n} \int d z_{m} d z_{n}^{\prime} c_{m, n}(q, x) \hat{\Phi}_{A}\left(z_{m}\right) \hat{\Phi}_{B}\left(z_{n}^{\prime}\right)
$$

(where $c_{m, n}$ are coefficients and $\Phi$ can be any of $A_{\mu}, \psi$ or $\bar{\psi}$ ) then

$$
W=\frac{1}{(2 \pi)^{4}} \int d^{4} x e^{-i q x} \sum_{m, n} \int d z_{m} c_{m, n}(q, x)\left\langle p_{A}\left|\hat{\Phi}_{A}\left(z_{m}\right)\right| p_{A}\right\rangle \int d z_{n}^{\prime}\left\langle p_{B}\left|\hat{\Phi}_{B}\left(z_{n}^{\prime}\right)\right| p_{B}\right\rangle .
$$

As we will demonstrate below, the relevant operators are quark and gluon fields with Wilson-line type gauge links collinear to either $p_{2}$ for $A$ fields or $p_{1}$ for $B$ fields.

\section{Power corrections and solution of classical YM equations}

\subsection{Power counting for background fields}

As we discussed in previous section, to get the hadronic tensor in the form (2.12) we need to calculate the functional integral (2.11) in the background of the fields (2.10). Since we integrate over fields (2.10) afterwards, we may assume that they satisfy Yang-Mills equations $^{4}$

$$
\begin{aligned}
i \not D_{A} \psi_{A} & =0, \quad D_{A}^{\nu} A_{\mu \nu}^{a}=g \sum_{f} \bar{\psi}_{A}^{f} \gamma_{\mu} t^{a} \psi_{A}^{f}, \\
i \not D_{B} \psi_{B} & =0, \quad D_{B}^{\nu} B_{\mu \nu}^{a}=g \sum_{f} \bar{\psi}_{B}^{f} \gamma_{\mu} t^{a} \psi_{B}^{f},
\end{aligned}
$$

where $A_{\mu \nu} \equiv \partial_{\mu} A_{\nu}-\partial_{\nu} A_{\mu}-i g\left[A_{\mu}, A_{\nu}\right], D_{A}^{\mu} \equiv\left(\partial^{\mu}-i g\left[A^{\mu},\right)\right.$ and similarly for $B$ fields.

It is convenient to choose a gauge where $A_{*}=0$ for projectile fields and $B_{\bullet}=0$ for target fields. The rotation from a general gauge (Feynman gauge in our case, see footnote 1 ) to this gauge is performed by the matrix $\Omega\left(x_{*}, x_{\bullet}, x_{\perp}\right)$ satisfying boundary conditions

$$
\Omega\left(x_{*}, x_{\bullet}, x_{\perp}\right) \stackrel{x_{*} \rightarrow-\infty}{\rightarrow}\left[x_{\bullet},-\infty\right]_{x}^{A_{*}}, \quad \Omega\left(x_{*}, x_{\bullet}, x_{\perp}\right) \stackrel{x_{\bullet} \rightarrow-\infty}{\rightarrow}\left[x_{*},-\infty_{*}\right]_{x}^{B \bullet}
$$

where $A_{*}\left(x_{\bullet}, x_{\perp}\right)$ and $B_{\bullet}\left(x_{*}, x_{\perp}\right)$ are projectile and target fields in an arbitrary gauge and $\left[x_{\bullet}, y_{\bullet}\right]_{z}^{A_{*}}$ denotes a gauge link constructed from $A$ fields ordered along a light-like line:

$$
\left[x_{\bullet}, y_{\bullet}\right]_{z}^{A_{*}}=P e^{\frac{2 i g}{s} \int_{y_{\bullet}}^{x} d z_{\bullet} A_{*}\left(z_{\bullet}, z_{\perp}\right)}
$$

and similarly for $\left[x_{*}, y_{*}\right]_{z}^{B \bullet}$.

The existence of matrix $\Omega\left(x_{*}, x_{\bullet}, x_{\perp}\right)$ was proved in appendix B of ref. [12] by explicit construction. The relative strength of Lorentz components of projectile and target fields

\footnotetext{
${ }^{4}$ As we mentioned, for the purpose of calculation of integral over $C$ fields the projectile and target fields are "frozen".
} 
in this gauge was found in ref. [12]

$$
\begin{aligned}
& \not p_{1} \psi_{A}\left(x_{\bullet}, x_{\perp}\right) \sim m_{\perp}^{5 / 2}, \quad \quad \gamma_{i} \psi_{A}\left(x_{\bullet}, x_{\perp}\right) \sim m_{\perp}^{3 / 2}, \quad \not p_{2} \psi_{A}\left(x_{\bullet}, x_{\perp}\right) \sim s \sqrt{m_{\perp}}, \\
& \not p_{1} \psi_{B}\left(x_{*}, x_{\perp}\right) \sim s \sqrt{m_{\perp}}, \quad \quad \gamma_{i} \psi_{B}\left(x_{*}, x_{\perp}\right) \sim m_{\perp}^{3 / 2}, \quad \not p_{2} \psi_{B}\left(x_{*}, x_{\perp}\right) \sim m_{\perp}^{5 / 2} \text {, } \\
& A_{\bullet}\left(x_{\bullet}, x_{\perp}\right) \sim B_{*}\left(x_{*}, x_{\perp}\right) \sim m_{\perp}^{2}, \quad A_{i}\left(x_{\bullet}, x_{\perp}\right) \sim B_{i}\left(x_{*}, x_{\perp}\right) \sim m_{\perp} .
\end{aligned}
$$

Here $m_{\perp}$ is a scale of order of $m_{N}$ or $q_{\perp}$. In general, we consider $W\left(p_{A}, p_{B}, q\right)$ in the region where $s, Q^{2} \gg q_{\perp}^{2}, m_{N}^{2}$, while the relation between $q_{\perp}^{2}$ and $m_{N}^{2}$ and between $Q^{2}$ and $s$ may be arbitrary. So, for the purpose of counting of powers of $s$, we will not distinguish between $s$ and $Q^{2}$ (although at the final step we will be able to tell the difference since our final expressions for power corrections will have either $s$ or $Q^{2}$ in denominators). Similarly, for the purpose of power counting we will not distinguish between $m_{N}$ and $q_{\perp}$ so we introduce $m_{\perp}$ which may be of order of $m_{N}$ or $q_{\perp}$ depending on matrix element.

Note also that in our gauge

$$
A_{i}\left(x_{\bullet}, x_{\perp}\right)=\frac{2}{s} \int_{-\infty}^{x \bullet} d x_{\bullet}^{\prime} A_{* i}\left(x_{\bullet}^{\prime}, x_{\perp}\right), \quad B_{i}\left(x_{*}, x_{\perp}\right)=\frac{2}{s} \int_{-\infty}^{x_{*}} d x_{*}^{\prime} B \bullet i\left(x_{*}^{\prime}, x_{\perp}\right),
$$

where $A_{* i} \equiv F_{* i}^{(A)}$ and $B_{\bullet} \equiv F_{\bullet i}^{(B)}$ are field strengths for $A$ and $B$ fields respectively.

Thus, to find TMD factorization formula with power corrections at the tree level we need to calculate the functional integral (2.5) in the background fields of the strength given by eqs. (3.4).

\subsection{Approximate solution of classical equations}

As we discussed in section 2, the calculation of the functional integral (2.11) over $C$-fields in the tree approximation reduces to finding fields $C_{\mu}$ and $\psi_{C}$ as solutions of Yang-Mills equations for the action $S_{C}=S_{\mathrm{QCD}}\left(C+A+B, \psi_{C}+\psi_{A}+\psi_{B}\right)-S_{\mathrm{QCD}}\left(A, \psi_{A}\right)-S_{\mathrm{QCD}}\left(B, \psi_{B}\right)$

$$
\begin{aligned}
(i \not \partial+g \not A+g \not B+g \not \subset)\left(\psi_{A}^{f}+\psi_{B}^{f}+\psi_{C}^{f}\right) & =0, \\
D^{\nu} F_{\mu \nu}^{a}(A+B+C) & =g \sum_{f}\left(\bar{\psi}_{A}^{f}+\bar{\psi}_{B}^{f}+\bar{\psi}_{C}^{f}\right) \gamma_{\mu} t^{a}\left(\psi_{A}^{f}+\psi_{B}^{f}+\psi_{C}^{f}\right) .
\end{aligned}
$$

As we discussed above, the solution of eq. (3.6) which we need corresponds to the sum of set of diagrams in background field $A+B$ with retarded Green functions, see figure 3 . The retarded Green functions (in the background-Feynman gauge) are defined as

$$
\begin{aligned}
\left(x\left|\frac{1}{\mathcal{P}^{2} g^{\mu \nu}+2 i g \mathcal{F}^{\mu \nu}+i \epsilon p_{0}}\right| y\right) \equiv & \left(x\left|\frac{1}{p^{2}+i \epsilon p_{0}}\right| y\right)-g\left(x\left|\frac{1}{p^{2}+i \epsilon p_{0}} \mathcal{O}_{\mu \nu} \frac{1}{p^{2}+i \epsilon p_{0}}\right| y\right) \\
& +g^{2}\left(x\left|\frac{1}{p^{2}+i \epsilon p_{0}} \mathcal{O}_{\mu \xi} \frac{1}{p^{2}+i \epsilon p_{0}} \mathcal{O}^{\xi} \frac{1}{p^{2}+i \epsilon p_{0}}\right| y\right)+\ldots,
\end{aligned}
$$

where

$$
\begin{aligned}
\mathcal{P}_{\mu} & \equiv i \partial_{\mu}+g A_{\mu}+g B_{\mu}, \quad \mathcal{F}_{\mu \nu}=\partial_{\mu}(A+B)_{\nu}-\mu \leftrightarrow \nu-i g\left[A_{\mu}+B_{\mu}, A_{\nu}+B_{\nu}\right], \\
\mathcal{O}_{\mu \nu} & \equiv\left(\left\{p^{\xi}, A_{\xi}+B_{\xi}\right\}+g(A+B)^{2}\right) g_{\mu \nu}+2 i \mathcal{F}_{\mu \nu}
\end{aligned}
$$

and similarly for quarks. 


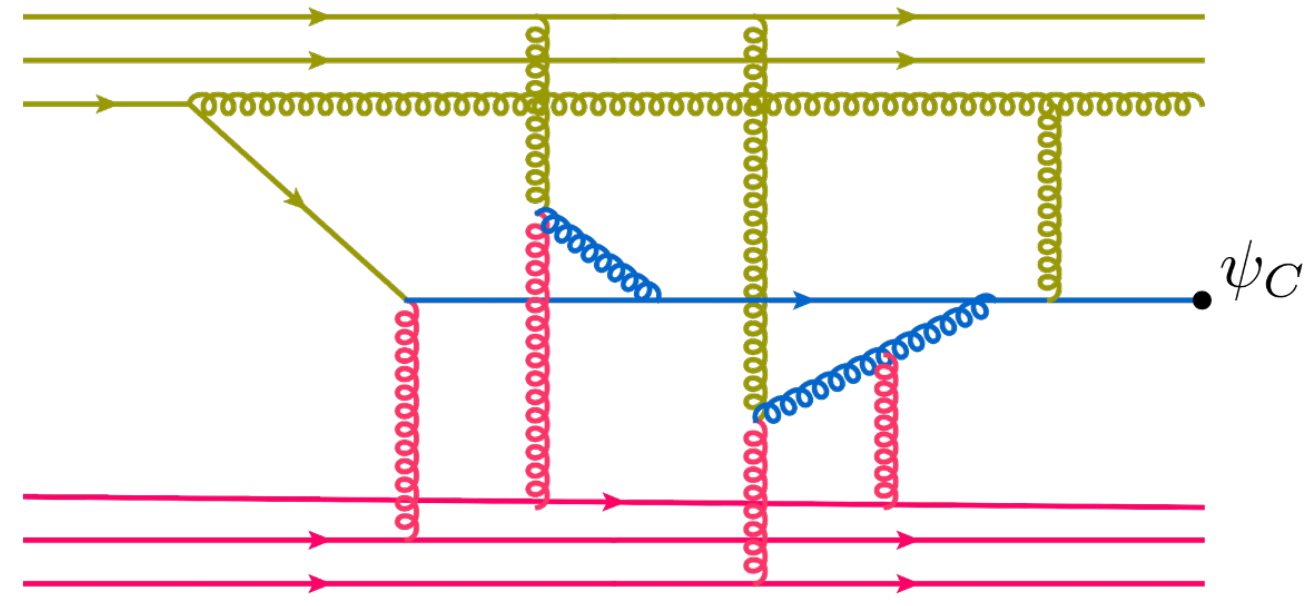

Figure 3. Typical diagram for the classical field with projectile/target sources. The Green functions of the central fields are given by retarded propagators.

Hereafter we use Schwinger's notations for propagators in external fields normalized according to

$$
(x|F(p)| y) \equiv \int d^{4} p e^{-i p(x-y)} F(p),
$$

where we use space-saving notation $d^{n} p \equiv \frac{d^{n} p}{(2 \pi)^{n}}$. Moreover, when it will not lead to a confusion, we will use short-hand notation

$$
\frac{1}{\mathcal{O}} \mathcal{O}^{\prime}(x) \equiv \int d^{4} z\left(x\left|\frac{1}{\mathcal{O}}\right| z\right) \mathcal{O}^{\prime}(z) .
$$

The solution of eqs. (3.6) in terms of retarded Green functions gives fields $C_{\mu}$ and $\psi_{C}$ that vanish at $t \rightarrow-\infty$. Thus, we are solving the usual classical YM equations ${ }^{5}$

$$
\mathbb{D}^{\nu} \mathbb{F}_{\mu \nu}^{a}=\sum_{f} g \bar{\Psi}^{f} t^{a} \gamma_{\mu} \Psi^{f}, \quad \not{P} \Psi^{f}=0
$$

where

$$
\begin{aligned}
\mathbb{A}_{\mu} & =C_{\mu}+A_{\mu}+B_{\mu}, & \Psi^{f} & =\psi_{C}^{f}+\psi_{A}^{f}+\psi_{B}^{f}, \\
\mathbb{P}_{\mu} & \equiv i \partial_{\mu}+g C_{\mu}+g A_{\mu}+g B_{\mu}, & \mathbb{F}_{\mu \nu} & =\partial_{\mu} \mathbb{A}_{\nu}-\mu \leftrightarrow \nu-i g\left[\mathbb{A}_{\mu}, \mathbb{A}_{\nu}\right],
\end{aligned}
$$

with boundary conditions ${ }^{6}$

$$
\begin{aligned}
& \mathbb{A}_{\mu}(x) \stackrel{x_{*}}{\stackrel{\rightarrow}{=}-\infty} A_{\mu}\left(x_{\bullet}, x_{\perp}\right), \quad \Psi(x) \stackrel{x_{*} \rightarrow-\infty}{=} \psi_{A}\left(x_{\bullet}, x_{\perp}\right), \\
& \mathbb{A}_{\mu}(x) \stackrel{x}{\stackrel{\rightarrow}{=}-\infty} B_{\mu}\left(x_{*}, x_{\perp}\right), \quad \Psi(x) \stackrel{x \bullet}{\stackrel{\rightarrow}{=}-\infty} \psi_{B}\left(x_{*}, x_{\perp}\right)
\end{aligned}
$$

following from $C_{\mu}, \psi_{C} \stackrel{t \rightarrow-\infty}{\rightarrow} 0$. These boundary conditions reflect the fact that at $t \rightarrow-\infty$ we have only incoming hadrons with $A$ and $B$ fields.

\footnotetext{
${ }^{5}$ We take into account only $u, d, s, c$ quarks and consider them massless.

${ }^{6} \mathrm{It}$ is convenient to fix redundant gauge transformations by requirements $A_{i}\left(-\infty_{\bullet}, x_{\perp}\right)=0$ for the projectile and $B_{i}\left(-\infty_{*}, x_{\perp}\right)=0$ for the target, see the discussion in ref. [30].
} 
As discussed in ref. [12], for our case of particle production with $\frac{q_{\perp}}{Q} \ll 1$ it is possible to find the approximate solution of (3.11) as a series in this small parameter. We will solve eqs. (3.11) iteratively, order by order in perturbation theory, starting from the zero-order approximation in the form of the sum of projectile and target fields

$$
\begin{aligned}
& \mathbb{A}_{\mu}^{[0]}(x)=A_{\mu}\left(x_{\bullet}, x_{\perp}\right)+B_{\mu}\left(x_{*}, x_{\perp}\right), \\
& \Psi^{[0]}(x)=\psi_{A}\left(x_{\bullet}, x_{\perp}\right)+\psi_{B}\left(x_{*}, x_{\perp}\right)
\end{aligned}
$$

and improving it by calculation of Feynman diagrams with retarded propagators in the background fields (3.14).

The first step is the calculation of the linear term for the trial configuration (3.14). The quark part of the linear term has the form

$$
\begin{aligned}
L_{\psi} & \equiv \not P \Psi^{[0]}=L_{\psi}^{(0)}+L_{\psi}^{(1)}, \\
L_{\psi}^{(0)} & =g \gamma^{i} A_{i} \psi_{B}+g \gamma^{i} B_{i} \psi_{A}, \quad L_{\psi}^{(1)}=\frac{2 g}{s} \not p_{2} A_{\bullet} \psi_{B}+\frac{2 g}{s} \not p_{1} B_{*} \psi_{A},
\end{aligned}
$$

where

$$
\mathcal{P}_{\bullet}=i \partial_{\bullet}+g A_{\bullet}, \quad \mathcal{P}_{*}=i \partial_{*}+g B_{*}, \quad \mathcal{P}_{i}=i \partial_{i}+g A_{i}+g B_{i}
$$

are operators in external zero-order fields (3.14). Here we denote the order of expansion in the parameter $\frac{m_{\perp}^{2}}{s}$ by $(\ldots)^{(n)}$, and the order of perturbative expansion is labeled by $(\ldots)^{[n]}$ as usual. The power-counting estimates for linear term in eq. (3.15) comes from eq. (3.4) in the form

$$
L_{\psi}^{(0)} \sim m_{\perp}^{5 / 2}, \quad L_{\psi}^{(1)} \sim \frac{m_{\perp}^{9 / 2}}{s} .
$$

The gluon linear term is

$$
\begin{aligned}
L_{\mu}^{a} & \equiv \mathcal{D}^{\xi} \mathcal{F}_{\xi \mu}^{a}+g \bar{\Psi}^{[0]} \gamma_{\mu} t^{a} \Psi^{[0]}=L_{\mu}^{(-1) a}+L_{\mu}^{(0) a}+L_{\mu}^{(1) a}, \\
L_{\mu}^{(-1) a} & =\frac{2 g}{s} p_{1 \mu} f^{a b c} A_{* j}^{b} B^{c j}+\frac{2 g}{s} p_{2 \mu} f^{a b c} B_{\bullet j}^{b} A^{c j} \sim s m_{\perp}^{2} .
\end{aligned}
$$

The explicit form of gluon linear terms $L_{\mu}^{(0) a}$ and $L_{\mu}^{(1) a}$ is presented in eq. (3.26) from our paper [12]. For our purposes we need only the leading term $L_{\mu}^{(-1) a}$.

With the linear terms (3.15) and (3.18), a couple of first terms in our perturbative series are

$$
\Psi^{[1]}(x)=-\int d^{4} z\left(x\left|\frac{1}{\not \mathcal{P}}\right| z\right) L_{\psi}(z), \quad \Psi^{[2]}(x)=-g \int d^{4} z\left(x\left|\frac{1}{\not \mathcal{P}}\right| z\right) \mathbb{A}^{[1]}(z) \Psi^{[0]}(z)
$$

for quark fields and

$$
\begin{aligned}
\mathbb{A}_{\mu}^{[1] a}(x)= & \int d^{4} z\left(x\left|\frac{1}{\mathcal{P}^{2} g^{\mu \nu}+2 i g \mathcal{F} \mu \nu}\right| z\right)^{a b} L^{b \nu}(z), \\
\mathbb{A}_{\mu}^{[2] a}(x)= & g \int d^{4} z\left[-i\left(x\left|\frac{1}{\mathcal{P}^{2} g^{\mu \eta}+2 i g \mathcal{F}^{\mu \eta}} \mathcal{P}^{\xi}\right| z\right)^{a a^{\prime}} f^{a^{\prime} b c} \mathbb{A}_{\xi}^{[1] b} \mathbb{A}^{[1] c \eta}\right. \\
& \left.+\left(x\left|\frac{1}{\mathcal{P}^{2} g^{\mu \eta}+2 i g \mathcal{F} \mu \eta}\right| z\right)^{a a^{\prime}} f^{a^{\prime} b c} \mathbb{A}^{[1] b \xi}\left(\mathcal{D}_{\xi} \mathbb{A}^{[1] c \eta}-\mathcal{D}^{\eta} \mathbb{A}_{\xi}^{[1] c}\right)\right]
\end{aligned}
$$


for gluon fields (in the background-Feynman gauge). Next iterations, like $\Psi^{[3]}(x)$ and $\mathbb{A}_{\mu}^{[3] a}(x)$, will give us a set of tree-level Feynman diagrams in the background fields $A_{\mu}+B_{\mu}$ and $\psi_{A}+\psi_{B}$.

Let us consider the fields in the first order in perturbative expansion:

$$
\begin{aligned}
\Psi^{[1]}=-\frac{1}{\not \mathcal{P}} L_{\psi} & =-\frac{1}{\alpha \not p_{1}+\beta \not p_{2}+\frac{2 g}{s}\left(B_{*} \not p_{1}+A_{\bullet} p_{2}\right)+\not{P}_{\perp}+i \epsilon p_{0}} L_{\psi}, \\
\mathbb{A}_{\mu}^{[1]} & =\frac{1}{\mathcal{P}^{2} g^{\mu \nu}+2 i g \mathcal{F} \mu \nu} L^{\nu} \\
& =\frac{1}{\left[\left\{\alpha+\frac{2 g}{s} B_{*}, \beta+\frac{2 g}{s} A_{\bullet}\right\} \frac{s}{2}-\mathcal{P}_{\perp}^{2}\right] g^{\mu \nu}+2 i g \mathcal{F}^{\mu \nu}+i \epsilon p_{0}} L^{\nu} .
\end{aligned}
$$

Here $\alpha, \beta$, and $p_{\perp}$ are understood as differential operators $\alpha=i \frac{\partial}{\partial x_{\bullet}}, \beta=i \frac{\partial}{\partial x_{*}}$ and $p_{i}=i \frac{\partial}{\partial x^{2}}$.

Now comes the central point of our approach. Let us expand quark and gluon propagators in powers of background fields, then we get a set of diagrams shown in figure 3. The typical bare gluon propagator in figure 3 is

$$
\frac{1}{p^{2}+i \epsilon p_{0}}=\frac{1}{\alpha \beta s-p_{\perp}^{2}+i \epsilon(\alpha+\beta)} .
$$

Since we do not consider loops of $C$-fields in this paper, the transverse momenta in tree diagrams are determined by further integration over projectile ("A") and target ("B") fields in eq. (2.8) which converge on either $q_{\perp}$ or $m_{N}$. On the other hand, the integrals over $\alpha$ converge on either $\alpha_{q}$ or $\alpha \sim 1$ and similarly the characteristic $\beta$ 's are either $\beta_{q}$ or $\beta \sim 1$. Since $\alpha_{q} \beta_{q} s=Q_{\|}^{2} \gg q_{\perp}^{2}$, one can expand gluon and quark propagators in powers of $\frac{p_{\perp}^{2}}{\alpha \beta s}$

$$
\begin{aligned}
\frac{1}{p^{2}+i \epsilon p_{0}} & =\frac{1}{s(\alpha+i \epsilon)(\beta+i \epsilon)}\left(1+\frac{p_{\perp}^{2} / s}{(\alpha+i \epsilon)(\beta+i \epsilon)}+\ldots\right) \\
\frac{\not p}{p^{2}+i \epsilon p_{0}} & =\frac{1}{s}\left(\frac{\not p_{1}}{\beta+i \epsilon}+\frac{\not p_{2}}{\alpha+i \epsilon}+\frac{\not p_{\perp}}{(\alpha+i \epsilon)(\beta+i \epsilon)}\right)\left(1+\frac{p_{\perp}^{2} / s}{(\alpha+i \epsilon)(\beta+i \epsilon)}+\ldots\right) .
\end{aligned}
$$

The explicit form of operators $\frac{1}{\alpha+i \epsilon}, \frac{1}{\beta+i \epsilon}$, and $\frac{1}{(\alpha+i \epsilon)(\beta+i \epsilon)}$ is

$$
\begin{aligned}
\left(x\left|\frac{1}{\alpha+i \epsilon}\right| y\right) & =\frac{s}{2} \int d^{2} p_{\perp} \int \frac{d \alpha}{\alpha+i \epsilon} d \beta e^{-i \alpha(x-y)_{\bullet}-i \beta(x-y)_{*}+i(p, x-y)_{\perp}} \\
& =-i \frac{s}{2}(2 \pi)^{2} \delta^{(2)}\left(x_{\perp}-y_{\perp}\right) \theta\left(x_{\bullet}-y_{\bullet}\right) \delta\left(x_{*}-y_{*}\right), \\
\left(x\left|\frac{1}{\beta+i \epsilon}\right| y\right) & =\frac{s}{2} \int d^{2} p_{\perp} \int d \alpha \frac{d \beta}{\beta+i \epsilon} e^{-i \alpha(x-y) \bullet-i \beta(x-y)_{*}+i(p, x-y)_{\perp}} \\
& =-i \frac{s}{2}(2 \pi)^{2} \delta^{(2)}\left(x_{\perp}-y_{\perp}\right) \theta\left(x_{*}-y_{*}\right) \delta\left(x_{\bullet}-y_{\bullet}\right), \\
\left(x\left|\frac{1}{(\alpha+i \epsilon)(\beta+i \epsilon)}\right| y\right) & =\frac{s}{2} \int d^{2} p_{\perp} \int \frac{d \alpha}{\alpha+i \epsilon} \frac{d \beta}{\beta+i \epsilon} e^{-i \alpha(x-y) \bullet-i \beta(x-y)_{*}+i(p, x-y)_{\perp}} \\
& =-\frac{s}{2}(2 \pi)^{2} \delta^{(2)}\left(x_{\perp}-y_{\perp}\right) \theta\left(x_{*}-y_{*}\right) \theta\left(x_{\bullet}-y_{\bullet}\right) .
\end{aligned}
$$


After the expansion (3.23), the dynamics in the transverse space effectively becomes trivial: all background fields stand either at $x$ or at 0 . The formula (3.21) turns into expansion

$$
\begin{aligned}
& \Psi_{f}^{[1]}=-\left(\frac{\not p_{1}}{\beta s}+\frac{\not p_{2}}{\alpha s}\right) L_{\psi}+\frac{2 g}{s^{2}}\left(B_{*} \frac{\not p_{2}}{\alpha^{2}}+A \bullet \frac{\not p_{1}}{\beta^{2}}\right) L_{\psi}+\frac{1}{s^{2}}\left(\frac{\not p_{1}}{\beta} \not{P}_{\perp} \frac{\not p_{2}}{\alpha}+\frac{\not p_{2}}{\alpha} \not \mathcal{P}_{\perp} \frac{\not p_{1}}{\beta}\right) L_{\psi}+\ldots, \\
& \mathbb{A}_{\mu}^{[1]}=\frac{1}{\alpha \beta s} L_{\mu}+\frac{1}{\alpha \beta s}\left(\left[\mathcal{P}_{\perp}^{2}-g\left\{\alpha, A_{\bullet}\right\}-g\left\{\beta, B_{*}\right\}\right] g_{\mu \nu}-2 i g \mathcal{F}_{\mu \nu}\right) \frac{1}{\alpha \beta s} L^{\nu}+\ldots,
\end{aligned}
$$

where $\frac{1}{\alpha}$ and $\frac{1}{\beta}$ are understood as $\frac{1}{\alpha+i \epsilon}$ and $\frac{1}{\beta+i \epsilon}$ respectively.

One may question why we do not cut the integrals in eq. (3.24) to $|\alpha|>\sigma_{b}$ and $|\beta|>\sigma_{a}$ according to the definition of $C$ fields in section $2 .^{7}$ The reason is that in the diagrams like figure 3 with retarded propagators (3.24) one can shift the contour of integration over $\alpha$ and/or $\beta$ to the complex plane away to avoid the region of small $\alpha$ or $\beta$. It should be mentioned, however, that such shift may not be possible if there is pinching of poles in the integrals over $\alpha$ or $\beta$. For example, if after the expansion (3.23) we encounter $\frac{1}{(\alpha+i \epsilon)(\alpha-i \epsilon)}$, the expansion was not justified since actual $\alpha$ 's in the integral are $\sim \frac{p_{\perp}^{2}}{s}$ and hence the field was misidentified: we have a propagator of $B$-field rather than of $C$-field. Fortunately, at the tree level all propagators are retarded and the pinching of poles never occurs. In the higher orders in perturbation theory Feynman propagators in the loops cannot be replaced by retarded propagators so after the expansion $(3.23)$ we can get $\frac{1}{(\alpha+i \epsilon \beta)\left(\alpha+i \epsilon \beta^{\prime}\right)}$. In such case the pinching may occur so one needs to formulate a subtraction program to get rid of pinched poles and avoid double counting of the fields.

Note that the background fields are also smaller than typical $p_{\|}^{2} \sim s$. Indeed, from eq. (3.4) we see that $p_{\bullet}=\frac{s}{2} \beta \gg A_{\bullet} \sim m_{\perp}^{2}$ ( because $\alpha \geq \alpha_{q} \gg \frac{m_{\perp}^{2}}{s}$ ) and similarly $p_{*} \gg B_{*}$. Also $\left(p_{i}+A_{i}+B_{i}\right)^{2} \sim q_{\perp}^{2} \ll p_{\| \cdot}^{2}{ }^{8}$

\subsection{Power expansion of classical quark fields}

Now we expand the classical quark fields in powers of $\frac{p_{\perp}^{2}}{p_{\|}^{2}} \sim \frac{m_{\perp}^{2}}{s}$ (the expansion of classical gluon fields is presented in eqs. (3.35)-(3.38) in ref. [12]). From the previous section it is clear that the leading power correction comes only from the first term displayed in eq. (3.19). Expanding it in powers of $p_{\perp}^{2} / p_{\|}^{2}$ as explained in the previous section, we obtain

$$
\Psi(x)=\Psi^{[0]}(x)+\Psi^{[1]}(x)+\Psi^{[2]}(x)+\cdots=\Psi_{A}^{(0)}+\Psi_{B}^{(0)}+\Psi_{A}^{(1)}+\Psi_{B}^{(1)}+\ldots,
$$

where

$$
\begin{array}{ll}
\Psi_{A}^{(0)}=\psi_{A}+\Xi_{2 A}, & \Xi_{2 A}=-\frac{g p_{2}}{s} \gamma^{i} B_{i} \frac{1}{\alpha+i \epsilon} \psi_{A}, \\
\bar{\Psi}_{A}^{(0)}=\bar{\psi}_{A}+\bar{\Xi}_{2 A}, & \bar{\Xi}_{2 A}=-\left(\bar{\psi}_{A} \frac{1}{\alpha-i \epsilon}\right) \gamma^{i} B_{i} \frac{g p_{2}}{s}, \\
\Psi_{B}^{(0)}=\psi_{B}+\Xi_{1 B}, & \Xi_{1 B}=-\frac{g \not p_{1}}{s} \gamma^{i} A_{i} \frac{1}{\beta+i \epsilon} \psi_{B}, \\
\bar{\Psi}_{B}^{(0)}=\bar{\psi}_{B}+\bar{\Xi}_{1 B}, & \bar{\Xi}_{1 B}=-\left(\bar{\psi}_{B} \frac{1}{\beta-i \epsilon}\right) \gamma^{i} A_{i} \frac{g p_{1}}{s} .
\end{array}
$$

\footnotetext{
${ }^{7}$ Such cutoffs for integrals over $C$ fields are introduced explicitly in the framework of soft-collinear effective theory (SCET), see review [31].

${ }^{8}$ The only exception is the fields $B_{\bullet}$ or $A_{* i}$ which are of order of $s m_{\perp}$ but we saw in ref. [12] that effectively the expansion in powers of these fields is cut at the second term.
} 
In this formula

$$
\begin{aligned}
\frac{1}{\alpha+i \epsilon} \psi_{A}\left(x_{\bullet}, x_{\perp}\right) & \equiv-i \int_{-\infty}^{x_{\bullet}} d x_{\bullet}^{\prime} \psi_{A}\left(x_{\bullet}^{\prime}, x_{\perp}\right), \\
\left(\bar{\psi}_{A} \frac{1}{\alpha-i \epsilon}\right)\left(x_{\bullet}, x_{\perp}\right) & \equiv i \int_{-\infty}^{x_{\bullet}} d x_{\bullet}^{\prime} \bar{\psi}_{A}\left(x_{\bullet}^{\prime}, x_{\perp}\right)
\end{aligned}
$$

and similarly for $\frac{1}{\beta \pm i \epsilon}$. From now on we will denote $\left(\bar{\psi}_{A} \frac{1}{\alpha}\right)(x) \equiv\left(\bar{\psi}_{A} \frac{1}{\alpha-i \epsilon}\right)(x)$ and $\left(\bar{\psi}_{B} \frac{1}{\beta}\right)(x) \equiv\left(\bar{\psi}_{B} \frac{1}{\beta-i \epsilon}\right)(x)$ while in all other places $\left(\frac{1}{\alpha} \mathcal{O}\right) \equiv\left(\frac{1}{\alpha+i \epsilon} \mathcal{O}\right)$ and $\left(\frac{1}{\beta} \mathcal{O}\right) \equiv\left(\frac{1}{\beta+i \epsilon} \mathcal{O}\right)$.

It is easy to see that power counting of these quark fields has the form

$$
\Psi_{A}^{(0)} \sim \Psi_{B}^{(0)} \sim m_{\perp}^{3 / 2}
$$

As to quark fields $\Psi^{(1)}$, we present their explicit form in appendix A and prove in appendix $\mathrm{C}$ that their contribution is small in the kinematic region $s \gg Q^{2}$.

\section{$4 \quad$ Leading power corrections at $s \gg Q^{2} \gg q_{\perp}^{2}$}

As we mentioned in the introduction, our method is relevant to calculation of power corrections at any $s, Q^{2} \gg q_{\perp}^{2}, m_{N}^{2}$. However, the expressions are greatly simplified in the physically interesting case $s \gg Q^{2} \gg q_{\perp}^{2}$ which we consider in this paper. ${ }^{9}$

As we noted above, we take into account only $u, d, s, c$ quarks and consider them massless. The hadronic tensor takes the form

$$
\begin{aligned}
W\left(p_{A}, p_{B}, q\right) & =\int d^{2} x_{\perp} e^{i(q, x)_{\perp}} W\left(\alpha_{q}, \beta_{q}, x_{\perp}\right), \\
W\left(\alpha_{q}, \beta_{q}, x_{\perp}\right) & \equiv \frac{1}{(2 \pi)^{4}} \frac{2}{s} \int d x_{\bullet} d x_{*} e^{-i \alpha_{q} x_{\bullet}-i \beta_{q} x_{*}}\left\langle p_{A}, p_{B}\left|J_{\mu}\left(x_{\bullet}, x_{*}, x_{\perp}\right) J^{\mu}(0)\right| p_{A}, p_{B}\right\rangle,
\end{aligned}
$$

where $\left(c_{W} \equiv \cos \theta_{W}, s_{W} \equiv \sin \theta_{W}\right)^{10}$

$$
\begin{aligned}
J_{\mu}=\frac{e}{4 s_{W} c_{W}}[ & -\bar{u} \gamma_{\mu}\left(1-\frac{8}{3} s_{W}^{2}-\gamma_{5}\right) u-\bar{c} \gamma_{\mu}\left(1-\frac{8}{3} s_{W}^{2}-\gamma_{5}\right) c \\
& \left.+\bar{d} \gamma_{\mu}\left(1-\frac{4}{3} s_{W}^{2}-\gamma_{5}\right) d+\bar{s} \gamma_{\mu}\left(1-\frac{4}{3} s_{W}^{2}-\gamma_{5}\right) s\right] .
\end{aligned}
$$

After integration over central fields in the tree approximation we obtain

$$
W\left(\alpha_{q}, \beta_{q}, x_{\perp}\right) \equiv \frac{2}{(2 \pi)^{4} s} \int d x_{\bullet} d x_{*} e^{-i \alpha_{q} x_{\bullet}-i \beta_{q} x_{*}}\left\langle p_{A}\left|\left\langle p_{B}\left|\mathcal{J}_{\mu}\left(x_{\bullet}, x_{*}, x_{\perp}\right) \mathcal{J}^{\mu}(0)\right| p_{A}\right\rangle\right| p_{B}\right\rangle,
$$

where

$$
\begin{aligned}
\mathcal{J}^{\mu} & =\mathcal{J}_{A}^{\mu}+\mathcal{J}_{B}^{\mu}+\mathcal{J}_{A B}^{\mu}+\mathcal{J}_{B A}^{\mu}, \\
\mathcal{J}_{A}^{\mu} & =\frac{e}{4 s_{W} c_{W}}\left[-\bar{\Psi}_{A u} \breve{\gamma}^{\mu} \Psi_{A u}-\bar{\Psi}_{A c} \breve{\gamma}^{\mu} \Psi_{A c}+\bar{\Psi}_{A d} \breve{\gamma}^{\mu} \Psi_{A d}+\bar{\Psi}_{A s} \breve{\gamma}^{\mu} \Psi_{A s}\right], \\
\mathcal{J}_{A B}^{\mu} & =\frac{e}{4 s_{W} c_{W}}\left[-\bar{\Psi}_{A u} \breve{\gamma}^{\mu} \Psi_{B u}-\bar{\Psi}_{A c} \breve{\gamma}^{\mu} \Psi_{B c}+\bar{\Psi}_{A d} \breve{\gamma}^{\mu} \Psi_{B d}+\bar{\Psi}_{A s} \breve{\gamma}^{\mu} \Psi_{B s}\right],
\end{aligned}
$$

\footnotetext{
${ }^{9}$ We also assume that Z-boson is emitted in the central region of rapidity so $\alpha_{q} s \sim \beta_{q} s \gg Q^{2}$.

${ }^{10} \mathrm{We}$ denote the weak coupling constant by $e / s_{W}$ and reserve the notation " $g$ " for QCD coupling constant.
} 
and similarly for $\mathcal{J}_{B}^{\mu}$ and $\mathcal{J}_{B A}^{\mu}$. Hereafter we use notation $\breve{\gamma}_{\mu} \equiv \gamma_{\mu}\left(a-\gamma_{5}\right)$ where $a$ is one of $a_{u, c}=\left(1-\frac{8}{3} s_{W}^{2}\right)$ or $a_{d, s}=\left(1-\frac{4}{3} s_{W}^{2}\right)$ depending on quark's flavor.

The quark fields are given by a series in the parameter $\frac{m_{\perp}^{2}}{s}$, see eqs. (3.27) and (A.2), where $\Psi$ can be any of $u, d, s$ or $c$ quarks. ${ }^{11}$ Accordingly, the currents (4.4) can be expressed as a series in this parameter, e.g.

$$
\begin{aligned}
\mathcal{J}_{A B}^{(0) \mu}= & \frac{e}{4 s_{W} c_{W}}\left[-\bar{\Psi}_{A u}^{(0)} \breve{\gamma}^{\mu} \Psi_{B u}^{(0)}-\bar{\Psi}_{A c}^{(0)} \breve{\gamma}^{\mu} \Psi_{B c}^{(0)}+\bar{\Psi}_{A d}^{(0)} \breve{\gamma}^{\mu} \Psi_{B d}^{(0)}+\bar{\Psi}_{A s}^{(0)} \breve{\gamma}^{\mu} \Psi_{B s}^{(0)}\right] \\
\mathcal{J}_{A B}^{(1) \mu}= & \frac{e}{4 s_{W} c_{W}}\left[-\bar{\Psi}_{A u}^{(1)} \breve{\gamma}^{\mu} \Psi_{B u}^{(0)}-\bar{\Psi}_{A u}^{c(0)} \breve{\gamma}^{\mu} \Psi_{B u}^{c(1)}-\bar{\Psi}_{A c}^{(1)} \breve{\gamma}^{\mu} \Psi_{B c}^{(0)}-\bar{\Psi}_{A c}^{(0)} \breve{\gamma}^{\mu} \Psi_{B c}^{(1)}\right. \\
& \left.+\bar{\Psi}_{A d}^{(1)} \breve{\gamma}^{\mu} \Psi_{B d}^{d(0)}+\bar{\Psi}_{A d}^{(0)} \breve{\gamma}^{\mu} \Psi_{B d}^{(1)}+\bar{\Psi}_{A s}^{(1)} \breve{\gamma}^{\mu} \Psi_{B s}^{(0)}+\bar{\Psi}_{A s}^{(0)} \breve{\gamma}^{\mu} \Psi_{B s}^{(1)}\right] .
\end{aligned}
$$

The leading power contribution comes only from product $\mathcal{J}_{A B}^{\mu}(x) \mathcal{J}_{B A \mu}(0)$ (or $\left.\mathcal{J}_{B A}^{\mu}(x) \mathcal{J}_{A B \mu}(0)\right)$, while power corrections may come from other terms like $\mathcal{J}_{A}^{\mu}(x) \mathcal{J}_{B \mu}(0)$. We will consider all terms in turn.

\subsection{Leading contribution and power corrections from $\mathcal{J}_{A B}^{\mu}(x) \mathcal{J}_{B A \mu}(0)$ terms}

Power expansion of $\mathcal{J}_{A B}^{\mu}(x) \mathcal{J}_{B A \mu}(0)$ reads

$$
\begin{aligned}
& \bar{\Psi}_{A}(x) \breve{\gamma}^{\mu} \Psi_{B}(x) \bar{\Psi}_{B}(0) \breve{\gamma}_{\mu} \Psi_{A}(0)=\bar{\Psi}_{A}^{(0)}(x) \breve{\gamma}^{\mu} \Psi_{B}^{(0)}(x) \bar{\Psi}_{B}^{(0)}(0) \breve{\gamma}_{\mu} \Psi_{A}^{(0)}(0) \\
& \quad+\bar{\Psi}_{A}^{(1)}(x) \breve{\gamma}^{\mu} \Psi_{B}^{(0)}(x) \bar{\Psi}_{B}^{(0)}(0) \breve{\gamma}_{\mu} \Psi_{A}^{(0)}(0)+\bar{\Psi}_{A}^{(0)}(x) \breve{\gamma}^{\mu} \Psi_{B}^{(1)}(x) \bar{\Psi}_{B}^{(0)}(0) \breve{\gamma}_{\mu} \Psi_{A}^{(0)}(0) \\
& \quad+\bar{\Psi}_{A}^{(0)}(x) \breve{\gamma}^{\mu} \Psi_{B}^{(0)}(x) \bar{\Psi}_{B}^{(1)}(0) \breve{\gamma}_{\mu} \Psi_{A}^{(0)}(0)+\bar{\Psi}_{A}^{(0)}(x) \breve{\gamma}^{\mu} \Psi_{B}^{(0)}(x) \bar{\Psi}_{B}^{(0)}(0) \breve{\gamma}_{\mu} \Psi_{A}^{(1)}(0)+\ldots
\end{aligned}
$$

In appendix C.4 we demonstrate that terms $\sim \Psi^{(1)}$ lead to power corrections $\sim \frac{q_{\perp}^{2}}{\alpha_{q} s}$ or $\sim \frac{q_{\perp}^{2}}{\beta_{q} s}$ which are much smaller than $\frac{q_{\perp}^{2}}{\alpha_{q} \beta_{q} s}=\frac{q_{\perp}^{2}}{Q_{\|}^{2}} \sim \frac{q_{\perp}^{2}}{Q^{2}}$ if Z-boson is emitted in the central region of rapidity. Note that since we want to calculate the leading power corrections, hereafter we substitute $Q_{\|}^{2}$ with $Q^{2}$. In the limit $s \gg Q^{2} \gg q_{\perp}^{2}$ this change of variables can only lead to errors of the order of subleading power terms.

As to terms $\sim \bar{\Psi}_{A}^{(0)}(x) \gamma_{\mu} \Psi_{B}^{(0)}(x) \bar{\Psi}_{B}^{(0)}(0) \gamma^{\mu} \Psi_{A}^{(0)}(0)$, they can be decomposed using eq. (3.27) as follows:

$$
\begin{aligned}
& {\left[\left(\bar{\psi}_{A}+\bar{\Xi}_{2 A}\right)(x) \breve{\gamma}_{\mu}\left(\psi_{B}+\Xi_{1 B}\right)(x)\right]\left[\left(\bar{\psi}_{B}+\bar{\Xi}_{1 B}\right)(0) \breve{\gamma}^{\mu}\left(\psi_{A}+\Xi_{2 A}\right)(0)\right]+x \leftrightarrow 0} \\
& =\left[\bar{\psi}_{A}(x) \breve{\gamma}_{\mu} \psi_{B}(x)\right]\left[\bar{\psi}_{B}(0) \breve{\gamma}^{\mu} \psi_{A}(0)\right] \\
& \quad+\left[\bar{\Xi}_{2 A}(x) \breve{\gamma}_{\mu} \psi_{B}(x)\right]\left[\bar{\psi}_{B}(0) \breve{\gamma}^{\mu} \psi_{A}(0)\right]+\left[\bar{\psi}_{A}(x) \breve{\gamma}_{\mu} \Xi_{1 B}(x)\right]\left[\bar{\psi}_{B}(0) \breve{\gamma}^{\mu} \psi_{A}(0)\right] \\
& \quad+\left[\bar{\psi}_{A}(x) \breve{\gamma}_{\mu} \psi_{B}(x)\right]\left[\bar{\Xi}_{1 B}(0) \breve{\gamma}^{\mu} \psi_{A}(0)\right]+\left[\bar{\psi}_{A}(x) \breve{\gamma}_{\mu} \psi_{B}(x)\right]\left[\bar{\psi}_{B}(0) \breve{\gamma}^{\mu} \Xi_{2 A}(0)\right] \\
& \quad+\left[\bar{\Xi}_{2 A}(x) \breve{\gamma}_{\mu} \psi_{B}(x)\right]\left[\bar{\psi}_{B}(0) \breve{\gamma}^{\mu} \Xi_{2 A}(0)\right]+\left[\bar{\psi}_{A}(x) \breve{\gamma}_{\mu} \Xi_{1 B}(x)\right]\left[\bar{\Xi}_{1 B}(0) \breve{\gamma}^{\mu} \psi_{A}(0)\right] \\
& \quad+\left[\bar{\Xi}_{2 A}(x) \breve{\gamma}_{\mu} \psi_{B}(x)\right]\left[\bar{\Xi}_{1 B}(0) \breve{\gamma}^{\mu} \psi_{A}(0)\right]+\left[\bar{\psi}_{A}(x) \breve{\gamma}_{\mu} \Xi_{1 B}(x)\right]\left[\bar{\psi}_{B}(0) \breve{\gamma}^{\mu} \Xi_{2 A}(0)\right] \\
& \quad+\left[\bar{\Xi}_{2 A}(x) \breve{\gamma}_{\mu} \Xi_{1 B}(x)\right]\left[\bar{\psi}_{B}(0) \breve{\gamma}^{\mu} \psi_{A}(0)\right]+\left[\bar{\psi}_{A}(x) \breve{\gamma}_{\mu} \psi_{B}(x)\right]\left[\bar{\Xi}_{1 B}(0) \breve{\gamma}^{\mu} \Xi_{2 A}(0)\right]+x \leftrightarrow 0 .
\end{aligned}
$$

First, let us consider the leading power term coming from the first term in the r.h.s. of this equation.

\footnotetext{
${ }^{11}$ As we mentioned, we will need only first two terms of the expansion given by eqs. (3.27) and (A.2).
} 


\subsection{Leading power contribution}

As we mentioned, the leading-power term comes from $\mathcal{J}_{A B}^{(0) \mu}(x) \mathcal{J}_{B A \mu}^{(0)}(0)$ and $\mathcal{J}_{B A}^{(0) \mu}(x) \mathcal{J}_{A B \mu}^{(0)}(0)$. Using Fierz transformation

$$
\begin{aligned}
\left(\bar{\psi}_{A} \gamma^{\mu}\left[a-\gamma_{5}\right] \chi_{A}\right)\left(\bar{\chi}_{B} \gamma_{\mu}\left[a-\gamma_{5}\right] \psi_{B}\right) & \\
= & \frac{1+a^{2}}{2}\left[\left(\bar{\psi}_{A} \gamma^{\alpha} \psi_{B}\right)\left(\bar{\chi}_{B} \gamma_{\alpha} \chi_{A}\right)+\left(\bar{\psi}_{A} \gamma^{\alpha} \gamma_{5} \psi_{B}\right)\left(\bar{\chi}_{B} \gamma_{\alpha} \gamma_{5} \chi_{A}\right)\right] \\
& -a\left[\left(\bar{\psi}_{A} \gamma^{\alpha} \psi_{B}\right)\left(\bar{\chi}_{B} \gamma_{\alpha} \gamma_{5} \chi_{A}\right)+\left(\bar{\psi}_{A} \gamma^{\alpha} \gamma_{5} \psi_{B}\right)\left(\bar{\chi}_{B} \gamma_{\alpha} \chi_{A}\right)\right] \\
& +\left(1-a^{2}\right)\left[\left(\bar{\psi}_{A} \psi_{B}\right)\left(\bar{\chi}_{B} \chi_{A}\right)-\left(\bar{\psi}_{A} \gamma_{5} \psi_{B}\right)\left(\bar{\chi}_{B} \gamma_{5} \chi_{A}\right)\right]
\end{aligned}
$$

with $a_{u, c}=\left(1-\frac{8}{3} s_{W}^{2}\right)$ and $a_{d, s}=\left(1-\frac{4}{3} s_{W}^{2}\right)$ one obtains

$$
\begin{aligned}
& N_{c} \frac{16 s_{W}^{2} c_{W}^{2}}{e^{2}}\left\langle p_{A}\left|\left\langle p_{B}\left|\mathcal{J}_{A B \mu}^{(0)}(x) \mathcal{J}_{B A}^{(0) \mu}(0)+(x \leftrightarrow 0)\right| p_{A}\right\rangle\right| p_{B}\right\rangle= \\
& =\left[\left\{\frac{1+a_{u}^{2}}{2}\left(\left\langle\bar{\psi}_{A u}(x) \gamma_{\mu} \psi_{A u}(0)\right\rangle\left\langle\bar{\psi}_{B u}(0) \gamma^{\mu} \psi_{B u}(x)\right\rangle+\gamma_{\mu} \otimes \gamma^{\mu} \leftrightarrow \gamma_{\mu} \gamma_{5} \otimes \gamma^{\mu} \gamma_{5}\right)\right.\right. \\
& \quad-a_{u}\left(\left\langle\bar{\psi}_{A u}(x) \gamma_{\mu} \psi_{A u}(0)\right\rangle\left\langle\bar{\psi}_{B u}(0) \gamma^{\mu} \gamma_{5} \psi_{B u}(x)\right\rangle+\gamma_{\mu} \otimes \gamma^{\mu} \gamma_{5} \leftrightarrow \gamma_{\mu} \gamma_{5} \otimes \gamma^{\mu}\right) \\
& \left.\quad+\left(1-a_{u}^{2}\right)\left(\left\langle\bar{\psi}_{A u}(x) \psi_{A u}(0)\right\rangle\left\langle\bar{\psi}_{B u}(0) \psi_{B u}(x)\right\rangle-\left\langle\bar{\psi}_{A u}(x) \gamma_{5} \psi_{A u}(0)\right\rangle\left\langle\bar{\psi}_{B u}(0) \gamma_{5} \psi_{B u}(x)\right\rangle\right)\right\} \\
& \quad+\{u \leftrightarrow c\}+\{u \leftrightarrow d\}+\{u \leftrightarrow s\}]+[x \leftrightarrow 0],
\end{aligned}
$$

where

$$
\left\langle\bar{\psi}_{A u}(x) \gamma_{\mu} \psi_{A u}(0)\right\rangle \equiv\left\langle A\left|\hat{\bar{\psi}}_{u}(x) \gamma_{\mu} \hat{\psi}_{u}(0)\right| A\right\rangle, \quad\left\langle\bar{\psi}_{B u}(x) \gamma_{\mu} \psi_{B u}(0)\right\rangle \equiv\left\langle B\left|\hat{\bar{\psi}}_{u}(x) \gamma_{\mu} \hat{\psi}_{u}(0)\right| B\right\rangle
$$

and similarly for other matrix elements (summation over color and Lorentz indices is implied).

As usual, after integration over background fields $A$ and $B$ we promote $A, \psi_{A}$ and $B, \psi_{B}$ to operators $\hat{A}, \hat{\psi}$. A subtle point is that our operators are not under T-product ordering so one should be careful while changing the order of operators in formulas like Fierz transformation. Fortunately, all our operators are separated either by space-like intervals or light-like intervals so they commute with each other.

In a general gauge for projectile and target fields these expressions read (see eq. (3.2))

$$
\begin{aligned}
\left\langle A\left|\hat{\bar{\psi}}_{f}(x) \gamma_{\mu} \hat{\psi}_{f}(0)\right| A\right\rangle & =\left\langle A\left|\hat{\bar{\psi}}_{f}\left(x_{\bullet}, x_{\perp}\right) \gamma_{\mu}\left[x_{\bullet},-\infty_{\bullet}\right]_{x}\left[x_{\perp}, 0_{\perp}\right]_{-\infty}\left[-\infty_{\bullet}, 0_{\bullet}\right]_{0} \hat{\psi}_{f}(0)\right| A\right\rangle, \\
\left\langle B\left|\hat{\bar{\psi}}_{f}(x) \gamma_{\mu} \hat{\psi}_{f}(0)\right| B\right\rangle & =\left\langle B\left|\hat{\bar{\psi}}_{f}\left(x_{*}, x_{\perp}\right) \gamma_{\mu}\left[x_{*},-\infty_{*}\right]_{x}\left[x_{\perp}, 0_{\perp}\right]_{-\infty *}\left[-\infty_{*}, 0_{*}\right]_{0} \hat{\psi}_{f}(0)\right| B\right\rangle
\end{aligned}
$$

and similarly for $\left\langle A\left|\hat{\bar{\psi}}_{f}(0) \gamma_{\mu} \hat{\psi}_{f}(x)\right| A\right\rangle$ and $\left\langle B\left|\hat{\bar{\psi}}_{f}(0) \gamma_{\mu} \hat{\psi}_{f}(x)\right| B\right\rangle$.

From parametrization of two-quark operators in section 4.2.1, it is clear that the leading power contribution to $W(q)$ of eq. (4.1) comes from the product of two $f_{1}^{\prime} s$ in eq. (4.13) and (4.15). It has the form [32]

$$
\begin{aligned}
W^{\mathrm{lt}}\left(\alpha_{q}, \beta_{q}, q_{\perp}\right)= & -\frac{e^{2}}{8 s_{W}^{2} c_{W}^{2} N_{c}} \int d^{2} k_{\perp}\left(\left\{( 1 + a _ { u } ^ { 2 } ) \left[f_{1}^{u}\left(\alpha_{q}, k_{\perp}\right) \bar{f}_{1}^{u}\left(\beta_{q}, q_{\perp}-k_{\perp}\right)\right.\right.\right. \\
& \left.\left.\left.+\bar{f}_{1}^{u}\left(\alpha_{q}, k_{\perp}\right) f_{1}^{u}\left(\beta_{q}, q_{\perp}-k_{\perp}\right)\right]\right\}+\{u \leftrightarrow c\}+\{u \leftrightarrow d\}+\{u \leftrightarrow s\}\right) .
\end{aligned}
$$


All other terms in the product of eqs. (4.13) and (4.15) give higher power contributions $\sim \frac{q_{\perp}^{2}}{s} W^{\mathrm{lt}}(q)$ (but $\left.\underline{\text { not }} \sim \frac{q_{\perp}^{2}}{Q^{2}} W^{\mathrm{lt}}(q)\right)^{12}$ so they can be neglected at $Q^{2} \ll s$. Similarly, the contribution of two matrix elements in eq. (4.17) is $\sim \frac{m_{\perp}^{2}}{s}$ in comparison to $W^{\mathrm{lt}}(q)$ so it can be neglected as well.

\subsubsection{Parametrization of leading matrix elements}

Let us first consider matrix elements of operators without $\gamma_{5}$. The standard parametrization of quark TMDs reads

$$
\begin{gathered}
\frac{1}{16 \pi^{3}} \int d x_{\bullet} d^{2} x_{\perp} e^{-i \alpha x_{\bullet}+i(k, x)_{\perp}}\left\langle A\left|\hat{\bar{\psi}}_{f}\left(x_{\bullet}, x_{\perp}\right) \gamma^{\mu} \hat{\psi}_{f}(0)\right| A\right\rangle \\
=p_{1}^{\mu} f_{1}^{f}\left(\alpha, k_{\perp}^{2}\right)+k_{\perp}^{\mu} f_{\perp}^{f}\left(\alpha, k_{\perp}^{2}\right)+p_{2}^{\mu} \frac{2 m_{N}^{2}}{s} f_{3}^{f}\left(\alpha, k_{\perp}^{2}\right), \\
\frac{1}{16 \pi^{3}} \int d x_{\bullet} d^{2} x_{\perp} e^{-i \alpha x_{\bullet}+i(k, x)_{\perp}}\left\langle A\left|\hat{\bar{\psi}}_{f}\left(x_{\bullet}, x_{\perp}\right) \hat{\psi}_{f}(0)\right| A\right\rangle=m_{N} e^{f}\left(\alpha, k_{\perp}^{2}\right)
\end{gathered}
$$

for quark distributions in the projectile and

$$
\begin{gathered}
\frac{1}{16 \pi^{3}} \int d x_{\bullet} d^{2} x_{\perp} e^{i \alpha x_{\bullet}-i(k, x)_{\perp}}\left\langle A\left|\hat{\bar{\psi}}_{f}\left(x_{\bullet}, x_{\perp}\right) \gamma^{\mu} \hat{\psi}_{f}(0)\right| A\right\rangle \\
=-p_{1}^{\mu} \bar{f}_{1}^{f}\left(\alpha, k_{\perp}^{2}\right)-k_{\perp}^{\mu} \bar{f}_{\perp}^{f}\left(\alpha, k_{\perp}^{2}\right)-p_{2}^{\mu} \frac{2 m_{N}^{2}}{s} \bar{f}_{3}^{f}\left(\alpha, k_{\perp}^{2}\right), \\
\frac{1}{16 \pi^{3}} \int d x_{\bullet} d^{2} x_{\perp} e^{i \alpha x_{\bullet}-i(k, x)_{\perp}}\left\langle A\left|\hat{\bar{\psi}}_{f}\left(x_{\bullet}, x_{\perp}\right) \hat{\psi}_{f}(0)\right| A\right\rangle=m_{N} \bar{e}^{f}\left(\alpha, k_{\perp}^{2}\right)
\end{gathered}
$$

for the antiquark distributions. ${ }^{13}$

The corresponding matrix elements for the target are obtained by trivial replacements $p_{1} \leftrightarrow p_{2}, x_{\bullet} \leftrightarrow x_{*}$ and $\alpha \leftrightarrow \beta$ :

$$
\begin{gathered}
\frac{1}{16 \pi^{3}} \int d x_{*} d^{2} x_{\perp} e^{-i \beta x_{*}+i(k, x)_{\perp}}\left\langle B\left|\hat{\bar{\psi}}_{f}\left(x_{*}, x_{\perp}\right) \gamma^{\mu} \hat{\psi}_{f}(0)\right| B\right\rangle \\
=p_{2}^{\mu} f_{1}^{f}\left(\beta, k_{\perp}^{2}\right)+k_{\perp}^{\mu} f_{\perp}^{f}\left(\beta, k_{\perp}^{2}\right)+p_{1}^{\mu} \frac{2 m_{N}^{2}}{s} f_{3}^{f}\left(\beta, k_{\perp}^{2}\right), \\
\frac{1}{16 \pi^{3}} \int d x_{*} d^{2} x_{\perp} e^{-i \beta x_{*}+i(k, x)_{\perp}}\left\langle B\left|\hat{\bar{\psi}}_{f}\left(x_{*}, x_{\perp}\right) \hat{\psi}_{f}(0)\right| B\right\rangle=m_{N} e^{f}\left(\beta, k_{\perp}^{2}\right),
\end{gathered}
$$

and

$$
\begin{aligned}
& \frac{1}{16 \pi^{3}} \int d x_{*} d^{2} x_{\perp} e^{i \beta x_{*}-i(k, x)_{\perp}}\left\langle B\left|\hat{\bar{\psi}}_{f}\left(x_{*}, x_{\perp}\right) \gamma^{\mu} \hat{\psi}_{f}(0)\right| B\right\rangle \\
& =-p_{2}^{\mu} \bar{f}_{1}^{f}\left(\beta, k_{\perp}^{2}\right)-k_{\perp}^{\mu} \bar{f}_{\perp}^{f}\left(\beta, k_{\perp}^{2}\right)-p_{1}^{\mu} \frac{2 m_{N}^{2}}{s} \bar{f}_{3}^{f}\left(\beta, k_{\perp}^{2}\right), \\
& \frac{1}{16 \pi^{3}} \int d x_{*} d^{2} x_{\perp} e^{i \beta x_{*}-i(k, x)_{\perp}}\left\langle B\left|\hat{\bar{\psi}}_{f}\left(x_{*}, x_{\perp}\right) \hat{\psi}_{f}(0)\right| B\right\rangle=m_{N} \bar{e}^{f}\left(\beta, k_{\perp}^{2}\right) .
\end{aligned}
$$

\footnotetext{
${ }^{12}$ The trivial but important point is that any $f\left(x, k_{\perp}\right)$ may have only logarithmic dependence on Bjorken $x$ but not the power dependence $\sim \frac{1}{x}$. Indeed, at small $x$ the cutoff of corresponding longitudinal integrals comes from the rapidity cutoff $\sigma_{a}$, see the discussion in section 2 . Thus, at small $x$ one can safely put $x=0$ and the corresponding logarithmic contributions would be proportional to powers of $\alpha_{s} \ln \sigma_{a}$ (or, in some cases, $\alpha_{s} \ln ^{2} \sigma_{a}$, see e.g. ref. [33]). Also, a more technical version of this argument was presented on page 12.

${ }^{13}$ In an arbitrary gauge, there are gauge links to $-\infty$ as displayed in eq. (4.11).
} 
Matrix elements of operators with $\gamma_{5}$ are parametrized as follows:

$$
\begin{aligned}
& \frac{1}{16 \pi^{3}} \int d x_{\bullet} d^{2} x_{\perp} e^{-i \alpha x_{\bullet}+i(k, x)_{\perp}}\left\langle A\left|\hat{\bar{\psi}}_{f}\left(x_{\bullet}, x_{\perp}\right) \gamma^{\mu} \gamma_{5} \hat{\psi}_{f}(0)\right| A\right\rangle=i \epsilon_{\mu \nu \lambda \rho} \frac{2}{s} p_{1}^{\nu} p_{2}^{\lambda} k^{\rho} g_{f}^{\perp}\left(\alpha, k_{\perp}^{2}\right), \\
& \frac{1}{16 \pi^{3}} \int d x_{\bullet} d^{2} x_{\perp} e^{i \alpha x_{\bullet}-i(k, x)_{\perp}}\left\langle A\left|\hat{\bar{\psi}}_{f}\left(x_{\bullet}, x_{\perp}\right) \gamma^{\mu} \gamma_{5} \hat{\psi}_{f}(0)\right| A\right\rangle=i \epsilon_{\mu \nu \lambda \rho} \frac{2}{s} p_{1}^{\nu} p_{2}^{\lambda} k^{\rho} \bar{g}_{f}^{\perp}\left(\alpha, k_{\perp}^{2}\right)
\end{aligned}
$$

The corresponding matrix elements for the target are obtained by trivial replacements $p_{1} \leftrightarrow p_{2}, x_{\bullet} \leftrightarrow x_{*}$ and $\alpha \leftrightarrow \beta$ similarly to eq. (4.16).

Finally, for future use we present the parametrization of time-odd TMDs

$$
\begin{aligned}
& \frac{1}{16 \pi^{3}} \int d x_{\bullet} d^{2} x_{\perp} e^{-i \alpha x_{\bullet}+i(k, x)_{\perp}}\left\langle A\left|\hat{\bar{\psi}}_{f}\left(x_{\bullet}, x_{\perp}\right) \sigma^{\mu \nu} \hat{\psi}_{f}(0)\right| A\right\rangle \\
&= \frac{1}{m_{N}}\left(k_{\perp}^{\mu} p_{1}^{\nu}-\mu \leftrightarrow \nu\right) h_{1 f}^{\perp}\left(\alpha, k_{\perp}^{2}\right)+\frac{2 m_{N}}{s}\left(p_{1}^{\mu} p_{2}^{\nu}-\mu \leftrightarrow \nu\right) h_{f}\left(\alpha, k_{\perp}^{2}\right) \\
& \quad+\frac{2 m_{N}}{s}\left(k_{\perp}^{\mu} p_{2}^{\nu}-\mu \leftrightarrow \nu\right) h_{3 f}^{\perp}\left(\alpha, k_{\perp}^{2}\right), \\
& \frac{1}{16 \pi^{3}} \int d x_{\bullet} d^{2} x_{\perp} e^{i \alpha x \bullet-i(k, x)_{\perp}}\left\langle A\left|\hat{\bar{\psi}}_{f}\left(x_{\bullet}, x_{\perp}\right) \sigma^{\mu \nu} \hat{\psi}_{f}(0)\right| A\right\rangle \\
&=-\frac{1}{m_{N}}\left(k_{\perp}^{\mu} p_{1}^{\nu}-\mu \leftrightarrow \nu\right) \bar{h}_{1 f}^{\perp}\left(\alpha, k_{\perp}^{2}\right)-\frac{2 m_{N}}{s}\left(p_{1}^{\mu} p_{2}^{\nu}-\mu \leftrightarrow \nu\right) \bar{h}_{f}\left(\alpha, k_{\perp}^{2}\right) \\
& \quad-\frac{2 m_{N}}{s}\left(k_{\perp}^{\mu} p_{2}^{\nu}-\mu \leftrightarrow \nu\right) \bar{h}_{3 f}^{\perp}\left(\alpha, k_{\perp}^{2}\right)
\end{aligned}
$$

and similarly for the target with usual replacements $p_{1} \leftrightarrow p_{2}, x_{\bullet} \leftrightarrow x_{*}$ and $\alpha \leftrightarrow \beta$.

Note that the coefficients in front of $f_{3}, g_{f}^{\perp}, h$ and $h_{3}^{\perp}$ in eqs. (4.13), (4.15), (4.17), and (4.18) contain an extra $\frac{1}{s}$ since $p_{2}^{\mu}$ enters only through the direction of gauge link so the result should not depend on rescaling $p_{2} \rightarrow \lambda p_{2}$. For this reason, these functions do not contribute to $W(q)$ in our approximation.

\subsection{Power corrections from $\mathcal{J}_{A B}^{\mu}(x) \mathcal{J}_{B A \mu}(0)$ terms}

The terms in eq. (4.7) proportional to $\Xi$ fields are

$$
\begin{aligned}
& {\left[\left(\bar{\psi}_{A}(x)+\bar{\Xi}_{2 A}(x)\right) \breve{\gamma}_{\mu}\left(\psi_{B}(x)+\Xi_{1 B}(x)\right)\right]} \\
& \quad \times\left[\left(\bar{\psi}_{B}(0)+\bar{\Xi}_{1 B}(0)\right) \breve{\gamma}^{\mu}\left(\psi_{A}(x)+\Xi_{2 A}(0)\right)\right]+x \leftrightarrow 0 \\
& \stackrel{\text { tw3 }}{=}\left[\bar{\Xi}_{2 A}(x) \breve{\gamma}_{\mu} \psi_{B}(x)\right]\left[\bar{\psi}_{B}(0) \breve{\gamma}^{\mu} \psi_{A}(0)\right]+\left[\bar{\psi}_{A}(x) \breve{\gamma}_{\mu} \Xi_{1 B}(x)\right]\left[\bar{\psi}_{B}(0) \breve{\gamma}^{\mu} \psi_{A}(0)\right] \\
& \quad+\left[\bar{\psi}_{A}(x) \breve{\gamma}_{\mu} \psi_{B}(x)\right]\left[\bar{\Xi}_{1 B}(0) \breve{\gamma}^{\mu} \psi_{A}(0)\right]+\left[\bar{\psi}_{A}(x) \breve{\gamma}_{\mu} \psi_{B}(x)\right]\left[\bar{\psi}_{B}(0) \breve{\gamma}^{\mu} \Xi_{2 A}(0)\right] \\
& \quad+\left[\bar{\Xi}_{2 A}(x) \breve{\gamma}_{\mu} \psi_{B}(x)\right]\left[\bar{\psi}_{B}(0) \breve{\gamma}^{\mu} \Xi_{2 A}(0)\right]+\left[\bar{\psi}_{A}(x) \breve{\gamma}_{\mu} \Xi_{1 B}(x)\right]\left[\bar{\Xi}_{1 B}(0) \breve{\gamma}^{\mu} \psi_{A}(0)\right] \\
& \quad+\left[\bar{\Xi}_{2 A}(x) \breve{\gamma}_{\mu} \psi_{B}(x)\right]\left[\bar{\Xi}_{1 B}(0) \breve{\gamma}^{\mu} \psi_{A}(0)\right]+\left[\bar{\psi}_{A}(x) \breve{\gamma}_{\mu} \Xi_{1 B}(x)\right]\left[\bar{\psi}_{B}(0) \breve{\gamma}^{\mu} \Xi_{2 A}(0)\right] \\
& \quad+\left[\bar{\Xi}_{2 A}(x) \breve{\gamma}_{\mu} \Xi_{1 B}(x)\right]\left[\bar{\psi}_{B}(0) \breve{\gamma}^{\mu} \psi_{A}(0)\right]+\left[\bar{\psi}_{A}(x) \breve{\gamma}_{\mu} \psi_{B}(x)\right]\left[\bar{\Xi}_{1 B}(0) \breve{\gamma}^{\mu} \Xi_{2 A}(0)\right]+x \leftrightarrow 0 .
\end{aligned}
$$

First, as we demonstrate in appendix C.1, the terms in the second, third, and fourth lines lead to negligible power corrections $\sim \frac{q_{\perp}^{2}}{\alpha_{q} s}$ or $\sim \frac{q_{\perp}^{2}}{\beta_{q} s}$, so we are left with contribution of the fifth and sixth lines. 


\subsubsection{Fifth line in eq. (4.19): the leading term in $\frac{1}{N_{c}}$}

Let us start with the term $\left[\bar{\psi}_{A}(x) \breve{\gamma}_{\mu} \Xi_{1 B}(x)\right]\left[\bar{\psi}_{B}(0) \breve{\gamma}^{\mu} \Xi_{2 A}(0)\right]$. Performing Fierz transformation (4.8) we obtain

$$
\begin{aligned}
{[} & \left.\bar{\psi}_{A}^{m}(x) \gamma_{\mu}\left(a-\gamma_{5}\right) \Xi_{1 B}^{m}(x)\right]\left[\bar{\psi}_{B}^{n}(0) \gamma^{\mu}\left(a-\gamma_{5}\right) \Xi_{2 A}^{n}(0)\right] \\
= & \frac{1+a^{2}}{2}\left\{\left[\bar{\psi}_{A}^{m}(x) \gamma_{\alpha} \Xi_{2 A}^{n}(0)\right]\left[\bar{\psi}_{B}^{n}(0) \gamma^{\alpha} \Xi_{1 B}^{m}(x)\right]+\left(\gamma_{\alpha} \otimes \gamma^{\alpha} \leftrightarrow \gamma_{\alpha} \gamma_{5} \otimes \gamma^{\alpha} \gamma_{5}\right)\right\} \\
& -a\left(\left[\bar{\psi}_{A}^{m}(x) \gamma_{\alpha} \Xi_{2 A}^{n}(0)\right]\left[\bar{\psi}_{B}^{n}(0) \gamma^{\alpha} \gamma_{5} \Xi_{1 B}^{m}(x)\right]+\left(\gamma_{\alpha} \otimes \gamma^{\alpha} \gamma_{5} \leftrightarrow \gamma_{\alpha} \gamma_{5} \otimes \gamma^{\alpha}\right)\right\} \\
& +\left(1-a^{2}\right)\left(\left[\bar{\psi}_{A}^{m}(x) \Xi_{2 A}^{n}(0)\right]\left[\bar{\psi}_{B}^{n}(0) \Xi_{1 B}^{m}(x)\right]-\left(1 \otimes 1 \leftrightarrow \gamma_{5} \otimes \gamma_{5}\right)\right\} \\
= & \frac{1+a^{2}}{2}\left\{\left[\bar{\psi}_{A}^{m}(x) \gamma_{i} \Xi_{2 A}^{n}(0)\right]\left[\bar{\psi}_{B}^{n}(0) \gamma^{i} \Xi_{1 B}^{m}(x)\right]+\left(\gamma_{i} \otimes \gamma^{i} \leftrightarrow \gamma_{i} \gamma_{5} \otimes \gamma^{i} \gamma_{5}\right)\right\} \\
& +\left(1-a^{2}\right)\left(\left[\bar{\psi}_{A}^{m}(x) \Xi_{2 A}^{n}(0)\right]\left[\bar{\psi}_{B}^{n}(0) \Xi_{1 B}^{m}(x)\right]-\left(1 \otimes 1 \leftrightarrow \gamma_{5} \otimes \gamma_{5}\right)\right\} \\
& -a\left(\left[\bar{\psi}_{A}^{m}(x) \gamma_{i} \Xi_{2 A}^{n}(0)\right]\left[\bar{\psi}_{B}^{n}(0) \gamma^{i} \gamma_{5} \Xi_{1 B}^{m}(x)\right]+\left(\gamma_{i} \otimes \gamma^{i} \gamma_{5} \leftrightarrow \gamma_{i} \gamma_{5} \otimes \gamma^{i}\right)\right\}+O\left(\frac{m_{\perp}^{8}}{s}\right) .
\end{aligned}
$$

Next, separating color-singlet contributions

$$
\begin{aligned}
\left\langle A, B\left|\left(\bar{\psi}_{A}^{m}\left(B_{j}\right)^{n k} \psi_{A}^{k}\right)\left(\bar{\psi}_{B}^{n}\left(A_{i}\right)^{m l} \psi_{B}^{l}\right)\right| A, B\right\rangle & =\left\langle A, B\left|\left(\bar{\psi}_{A}^{m}\left(A_{i}\right)^{m l} \psi_{A}^{k}\right)\left(\bar{\psi}_{B}^{n}\left(B_{j}\right)^{n k} \psi_{B}^{l}\right)\right| A, B\right\rangle \\
& =\frac{1}{N_{c}}\left\langle A\left|\left(\bar{\psi}_{A}^{m} A_{i}^{m l} \psi_{A}^{l}\right)\right| A\right\rangle\left\langle B\left|\left(\bar{\psi}_{B}^{n} B_{j}^{n k} \psi_{B}^{k}\right)\right| B\right\rangle
\end{aligned}
$$

we get

$$
\begin{aligned}
s^{2} N_{c} g^{-2}\left[\bar{\psi}_{A}(x) \breve{\gamma}_{\mu} \Xi_{1 B}(x)\right]\left[\bar{\psi}_{B}(0) \breve{\gamma}^{\mu} \Xi_{2 A}(0)\right] \\
=\frac{1+a^{2}}{2}\left\{\left[\bar{\psi}_{A}(x) A_{k}(x) \gamma_{i} \not p_{2} \gamma^{j} \frac{1}{\alpha} \psi_{A}(0)\right]\left[\bar{\psi}_{B}(0) B_{j}(0) \gamma^{i} \not p_{1} \gamma^{k} \frac{1}{\beta} \psi_{B}(x)\right]+\left(\gamma_{i} \otimes \gamma^{i} \leftrightarrow \gamma_{i} \gamma_{5} \otimes \gamma^{i} \gamma_{5}\right)\right\} \\
+\left(1-a^{2}\right)\left\{\left[\bar{\psi}_{A}(x) A_{k}(x) \not p_{2} \gamma^{j} \frac{1}{\alpha} \psi_{A}(0)\right]\left[\bar{\psi}_{B}(0) B_{j}(0) \not p_{1} \gamma^{k} \frac{1}{\beta} \psi_{B}(x)\right]-\left(\gamma^{j} \otimes \gamma^{k} \leftrightarrow \gamma^{j} \gamma_{5} \otimes \gamma^{k} \gamma_{5}\right)\right\} \\
\quad-a\left\{\left[\bar{\psi}_{A}(x) A_{k}(x) \gamma_{i} \gamma_{5} \not p_{2} \gamma^{j} \frac{1}{\alpha} \psi_{A}(0)\right]\left[\bar{\psi}_{B}(0) B_{j}(0) \gamma^{i} \not p_{1} \gamma^{k} \frac{1}{\beta} \psi_{B}(x)\right]+\left(\gamma_{i} \gamma_{5} \otimes \gamma^{i} \leftrightarrow \gamma_{i} \otimes \gamma^{i} \gamma_{5}\right)\right\} .
\end{aligned}
$$

Using equations (B.3), (B.4), and (B.8) from appendix B we can rewrite eq. (4.22) as

$$
\begin{aligned}
g^{-2} & N_{c}\left\{\bar{\psi}_{A}(x) \gamma_{\mu}\left(a-\gamma_{5}\right) \Xi_{1 B}(x)\right\}\left\{\bar{\psi}_{B}(0) \gamma^{\mu}\left(a-\gamma_{5}\right) \Xi_{2 A}(0)\right\} \\
= & \frac{1+a^{2}}{s^{2}}\left\{\bar{\psi}_{A}(x) \not p_{2}\left[A_{i}(x)-i \gamma_{5} \tilde{A}_{i}(x)\right] \frac{1}{\alpha} \psi_{A}(0)\right\}\left\{\bar{\psi}_{B}(0) \not p_{1}\left[B^{i}(0)-i \gamma_{5} \tilde{B}^{i}(0)\right] \frac{1}{\beta} \psi_{B}(x)\right\} \\
+ & \frac{1-a^{2}}{s^{2}}\left\{\bar{\psi}_{A}(x) A_{k}(x) \not p_{2} \gamma_{j} \frac{1}{\alpha} \psi_{A}(0)\right\} \\
& \times\left\{\bar{\psi}_{B}(0)\left[B^{j}(0) \not p_{1} \gamma^{k}-j \leftrightarrow k+g^{j k} B^{i}(0) \not p_{1} \gamma_{i}\right] \frac{1}{\beta} \psi_{B}(x)\right\} \\
- & \frac{2 a}{s^{2}}\left\{\bar{\psi}_{A}(x) \not p_{2}\left[\gamma_{5} A_{i}(x)-i \tilde{A}_{i}(x)\right] \frac{1}{\alpha} \psi_{A}(0)\right\} \\
& \times\left\{\bar{\psi}_{B}(0) \not p_{1}\left[B^{i}(0)-i \gamma_{5} \tilde{B}^{i}(0)\right] \frac{1}{\beta} \psi_{B}(x)\right\}+O\left(\frac{m_{\perp}^{8}}{s}\right) .
\end{aligned}
$$


For forward matrix elements we get

$$
\begin{aligned}
& \int d x_{\bullet} e^{-i \alpha_{q} x_{\bullet}}\left\langle A\left|\hat{\bar{\psi}}\left(x_{\bullet}, x_{\perp}\right) \not p_{2}\left[\hat{A}_{i}\left(x_{\bullet}, x_{\perp}\right)-i \gamma_{5} \hat{\tilde{A}}_{i}\left(x_{\bullet}, x_{\perp}\right)\right] \frac{1}{\alpha} \hat{\psi}(0)\right| A\right\rangle \\
& =\frac{1}{\alpha_{q}} \int d x_{\bullet} e^{-i \alpha_{q} x_{\bullet}}\left\langle A\left|\hat{\bar{\psi}}\left(x_{\bullet}, x_{\perp}\right) \not p_{2}\left[\hat{A}_{i}\left(x_{\bullet}, x_{\perp}\right)-i \gamma_{5} \hat{\tilde{A}}_{i}\left(x_{\bullet}, x_{\perp}\right)\right] \hat{\psi}(0)\right| A\right\rangle, \\
& \int d x_{*} e^{-i \beta_{q} x_{*}}\left\langle B\left|\hat{\bar{\psi}}(0) \not p_{1}\left[\hat{A}_{j}(0)-i \gamma_{5} \hat{\tilde{A}}_{j}(0)\right] \frac{1}{\beta} \hat{\psi}\left(x_{*}, x_{\perp}\right)\right| B\right\rangle \\
& =-\frac{1}{\beta_{q}} \int d x_{*} e^{-i \beta_{q} x_{*}}\left\langle B\left|\hat{\bar{\psi}}(0) \not p_{1}\left[\hat{A}_{j}(0)-i \gamma_{5} \hat{\tilde{A}}_{j}(0)\right] \hat{\psi}\left(x_{*}, x_{\perp}\right)\right| B\right\rangle
\end{aligned}
$$

and similarly for other Lorentz structures in eq. (4.23). The corresponding contribution of the r.h.s. of eq. (4.23) to $W\left(\alpha_{q}, \beta_{q}, x_{\perp}\right)$ takes the form ${ }^{14}$

$$
\begin{aligned}
- & \frac{e^{2} g^{2}(2 \pi)^{-4}}{8 s_{W}^{2} c_{W}^{2} N_{c} Q^{2}} \int d x_{\bullet} d x_{*} e^{-i \alpha_{q} x_{\bullet}-i \beta_{q} x_{*}}\left\{\frac{1+a^{2}}{s^{2}}\left\langle A\left|\hat{\bar{\psi}}\left(x_{\bullet}, x_{\perp}\right) \not p_{2}\left(\hat{A}_{i}-i \gamma_{5} \hat{\tilde{A}}_{i}\right)\left(x_{\bullet}, x_{\perp}\right) \hat{\psi}(0)\right| A\right\rangle\right. \\
& \times\left\langle B\left|\hat{\bar{\psi}}(0) \not p_{1}\left(\hat{A}^{i}-i \gamma_{5} \hat{\tilde{A}}^{i}\right)(0) \hat{\psi}\left(x_{*}, x_{\perp}\right)\right| B\right\rangle+\frac{1-a^{2}}{s^{2}}\left\langle A\left|\hat{\bar{\psi}}\left(x_{\bullet}, x_{\perp}\right) \hat{A}^{j}\left(x_{\bullet}, x_{\perp}\right) \not p_{2} \gamma_{j} \hat{\psi}(0)\right| A\right\rangle \\
& \left.\times\left\langle B\left|\hat{\bar{\psi}}(0) \hat{A}^{k}(0) \not p_{1} \gamma_{k} \hat{\psi}\left(x_{*}, x_{\perp}\right)\right| B\right\rangle\right\}\left(1+O\left(\frac{m_{\perp}^{2}}{s}\right)\right) .
\end{aligned}
$$

Note that for unpolarized hadrons $\left\langle B\left|\hat{\bar{\psi}}(0)\left(\hat{A}^{j}(0) \not p_{1} \gamma^{k}-j \leftrightarrow k\right) \hat{\psi}\left(x_{*}, x_{\perp}\right)\right| B\right\rangle=0$. Also, it is easy to see that the last line of eq. (4.23)

$$
-\frac{2 a}{s^{2}}\left\langle A\left|\hat{\bar{\psi}}(x) \not p_{2}\left[\hat{A}_{i}(x)-i \gamma_{5} \hat{\tilde{A}}_{i}(x)\right] \hat{\psi}(0)\right| A\right\rangle\left\langle B\left|\hat{\bar{\psi}}(0) \not p_{1}\left(\gamma_{5} \hat{A}^{i}(0)-i \hat{\tilde{A}}^{i}(0)\right] \hat{\psi}(x)\right| B\right\rangle
$$

gives zero contribution. Indeed, let us consider the first term in the r.h.s. of this equation. Since

$$
\begin{aligned}
& \left\langle A\left|\hat{\bar{\psi}}(x) \not p_{2}\left[\hat{A}_{i}(x)-i \gamma_{5} \hat{\tilde{A}}_{i}(x)\right] \hat{\psi}(0)\right| A\right\rangle \sim x_{i}, \\
& \left\langle B\left|\hat{\bar{\psi}}(0) \not p_{1}\left(\gamma_{5} \hat{A}^{i}(0)-i \hat{\tilde{A}}^{i}(0)\right] \hat{\psi}(x)\right| B\right\rangle \sim \epsilon^{i j} x_{j},
\end{aligned}
$$

this term vanishes (and similarly all other terms in the r.h.s. of eq. (4.26) do vanish too).

Repeating the same steps for the second term in the fifth line in eq. (4.19) we get

$$
\begin{aligned}
N_{c} g^{-2}\left\{\bar{\Xi}_{2 A}(x) \gamma_{\mu}\left(a-\gamma_{5}\right) \psi_{B}(x)\right\}\left\{\bar{\Xi}_{1 B}(0) \gamma^{\mu}\left(a-\gamma_{5}\right) \psi_{A}(0)\right\} \\
=\frac{1+a^{2}}{s^{2}}\left\{\left(\bar{\psi}_{A} \frac{1}{\alpha}\right)(x) \not p_{2}\left[A_{i}(0)+i \gamma_{5} \tilde{A}_{i}(0)\right] \psi_{A}(0)\right\}\left\{\left(\bar{\psi}_{B} \frac{1}{\beta}\right)(0) \not p_{1}\left[B^{i}(x)+i \gamma_{5} \tilde{B}^{i}(x)\right] \psi_{B}(x)\right\} \\
+\frac{1-a^{2}}{s^{2}}\left\{\left(\bar{\psi}_{A} \frac{1}{\alpha}\right)(x) A_{k}(0) \not p_{2} \gamma_{j} \psi_{A}(0)\right\} \\
\quad \times\left\{\left(\bar{\psi}_{B} \frac{1}{\beta}\right)(0)\left[B^{j}(x) \not p_{1} \gamma^{k}-j \leftrightarrow k+g^{j k} B^{i}(x) \not p_{1} \gamma_{i}\right] \psi_{B}(x)\right\} \\
-\frac{2 a}{s^{2}}\left\{\left(\bar{\psi}_{A} \frac{1}{\alpha}\right)(x) \not p_{2}\left[\gamma_{5} A_{i}(0)+i \tilde{A}_{i}(0)\right] \psi_{A}(0)\right\} \\
\quad \times\left\{\left(\bar{\psi}_{B} \frac{1}{\beta}\right)(0) \not p_{1}\left[B^{i}(x)+i \gamma_{5} \tilde{B}^{i}(x)\right] \psi_{B}(x)\right\}+O\left(\frac{m_{\perp}^{8}}{s}\right) .
\end{aligned}
$$

\footnotetext{
${ }^{14}$ After specifying the projectile and target matrix elements the " $A$ " and " $B$ " labels of the fields become redundant.
} 
Hereafter $\left(\bar{\psi}_{A} \frac{1}{\alpha}\right)(x) \equiv\left(\bar{\psi}_{A} \frac{1}{\alpha-i \epsilon}\right)(x)$ and $\left(\bar{\psi}_{B} \frac{1}{\beta}\right)(x) \equiv\left(\bar{\psi}_{B} \frac{1}{\beta-i \epsilon}\right)(x)$ (see eq. (3.28)) while in all other places $\left(\frac{1}{\alpha} \mathcal{O}\right) \equiv\left(\frac{1}{\alpha+i \epsilon} \mathcal{O}\right)$ and $\left(\frac{1}{\beta} \mathcal{O}\right) \equiv\left(\frac{1}{\beta+i \epsilon} \mathcal{O}\right)$.

For forward matrix elements this gives

$$
\begin{aligned}
& \int d x_{\bullet} e^{-i \alpha_{q} x_{\bullet}}\left\langle A\left|\left(\hat{\bar{\psi}} \frac{1}{\alpha}\right)\left(x_{\bullet}, x_{\perp}\right) \not p_{2}\left[\hat{A}_{i}(0)+i \gamma_{5} \hat{\tilde{A}}_{i}(0)\right] \psi(0)\right| A\right\rangle \\
& =\frac{1}{\alpha_{q}} \int d x_{\bullet} e^{-i \alpha_{q} x}\left\langle A\left|\hat{\bar{\psi}}\left(x_{\bullet}, x_{\perp}\right) \not p_{2}\left[\hat{A}_{i}(0)+i \gamma_{5} \hat{\tilde{A}}_{i}(0)\right] \psi(0)\right| A\right\rangle \\
& \int d x_{*} e^{-i \beta_{q} x_{*}}\left\langle B\left|\left(\hat{\bar{\psi}} \frac{1}{\beta}\right)(0) \not p_{1}\left[\hat{A}_{j}\left(x_{*}, x_{\perp}\right)+i \gamma_{5} \hat{\tilde{A}}_{j}\left(x_{*}, x_{\perp}\right)\right] \hat{\psi}\left(x_{*}, x_{\perp}\right)\right| B\right\rangle \\
& =-\frac{1}{\beta_{q}} \int d x_{*} e^{-i \beta_{q} x_{*}}\left\langle B\left|\hat{\bar{\psi}}(0) \not p_{1}\left[\hat{A}_{j}\left(x_{*}, x_{\perp}\right)+i \gamma_{5} \hat{\tilde{A}}_{j}\left(x_{*}, x_{\perp}\right)\right] \hat{\psi}\left(x_{*}, x_{\perp}\right)\right| B\right\rangle,
\end{aligned}
$$

and similarly for other Lorentz structures in eq. (4.28). Similarly to eq. (4.25), we get the contribution to $W\left(\alpha_{q}, \beta_{q}, x_{\perp}\right)$ in the form

$$
\begin{aligned}
& -\frac{e^{2} g^{2}}{8(2 \pi)^{4} s_{W}^{2} c_{W}^{2} N_{c} Q^{2}} \int d x_{\bullet} d x_{*} e^{-i \alpha_{q} x_{\bullet}-i \beta_{q} x_{*}} \\
& \quad \times\left\{\frac{1+a^{2}}{s^{2}}\left\langle A\left|\hat{\bar{\psi}}\left(x_{\bullet}, x_{\perp}\right) \not p_{2}\left[\hat{A}_{i}(0)+i \gamma_{5} \hat{\tilde{A}}_{i}(0)\right] \hat{\psi}(0)\right| A\right\rangle\right. \\
& \quad \times\left\langle B\left|\hat{\bar{\psi}}(0) \not p_{1}\left[\hat{A}^{i}\left(x_{*}, x_{\perp}\right)+i \gamma_{5} \hat{\tilde{A}}^{i}\left(x_{*}, x_{\perp}\right)\right] \hat{\psi}\left(x_{*}, x_{\perp}\right)\right| B\right\rangle \\
& \quad+\frac{1-a^{2}}{s^{2}}\left\langle A\left|\hat{\bar{\psi}}\left(x_{\bullet}, x_{\perp}\right) \hat{A}_{j}(0) \not p_{2} \gamma^{j} \hat{\psi}(0)\right| A\right\rangle \\
& \left.\quad \times\left\langle B\left|\hat{\bar{\psi}}(0) \hat{A}^{k}\left(x_{*}, x_{\perp}\right) \not p_{1} \gamma_{k} \hat{\psi}\left(x_{*}, x_{\perp}\right)\right| B\right\rangle\right\}\left(1+O\left(\frac{m_{\perp}^{2}}{s}\right)\right) .
\end{aligned}
$$

In section 4.3.2, we demonstrated that the matrix elements of quark-antiquark-gluon operators in eqs. (4.25) and (4.30) reduce to the leading-power TMDs from section 4.2.1. Using parametrizations from section 4.3 .2 we obtain the contribution of the 5 th line in eq. (4.19) to $W(q)$ in the form:

$$
\begin{aligned}
& W^{5 \operatorname{th}}\left(\alpha_{q}, \beta_{q}, q_{\perp}\right) \\
& =\frac{e^{2}}{4 s_{W}^{2} c_{W}^{2} N_{c} Q^{2}} \int d^{2} k_{\perp}\left[\left\{\left(1+a_{u}^{2}\right)(k, q-k)_{\perp} f_{1 u}\left(\alpha_{q}, k_{\perp}\right) \bar{f}_{1 u}\left(\beta_{q}, q_{\perp}-k_{\perp}\right)\right.\right. \\
& \left.+\frac{1}{m_{N}^{2}}\left(1-a_{u}^{2}\right) k_{\perp}^{2}(q-k)_{\perp}^{2} h_{1 u}^{\perp}\left(\alpha_{q}, k_{\perp}\right) \bar{h}_{1 u}^{\perp}\left(\beta_{q}, q_{\perp}-k_{\perp}\right)+\left(\alpha_{q} \leftrightarrow \beta_{q}\right)\right\} \\
& +\{u \leftrightarrow c\}+\{u \leftrightarrow d\}+\{u \leftrightarrow s\}]\left(1+O\left(\frac{m_{\perp}^{2}}{s}\right)\right),
\end{aligned}
$$

where quark $\leftrightarrow$ antiquark $\left(\alpha_{q} \leftrightarrow \beta_{q}\right)$ term comes from $x \leftrightarrow 0$ contribution in eq. (4.19). As we will demonstrate later, the power corrections which reduce to the leading-power TMDs come with the leading power of $\frac{1}{N_{c}}$ in the large- $N_{c}$ approximation - all other power corrections are $\sim \frac{1}{N_{c}^{2}}$ or $\frac{1}{N_{c}^{3}}$. 


\subsubsection{Parametrization of matrix elements from section 4.3.1}

In this section we will demonstrate that matrix elements of quark-antiquark-gluon operators from section 4.3.1 can be expressed in terms of leading-power matrix elements from section 4.2.1. Let us start with matrix element (4.24) which can be rewritten as (see ref. [22])

$$
\begin{aligned}
& g \int d x_{\bullet} d x_{\perp} e^{-i \alpha_{q} x_{\bullet}+i(k, x)_{\perp}}\left\langle A\left|\hat{\bar{\psi}}\left(x_{\bullet}, x_{\perp}\right) \not p_{2}\left[\hat{A}_{i}\left(x_{\bullet}, x_{\perp}\right)-i \gamma_{5} \hat{\tilde{A}}_{i}\left(x_{\bullet}, x_{\perp}\right)\right] \hat{\psi}(0)\right| A\right\rangle \\
& =\int d x_{\bullet} d x_{\perp} e^{-i \alpha_{q} x_{\bullet}+i(k, x)_{\perp}} \\
& \quad \times\left[k^{j}\left\langle A\left|\hat{\bar{\psi}}\left(x_{\bullet}, x_{\perp}\right) \gamma_{j} \not p_{2} \gamma_{i} \hat{\psi}(0)\right| A\right\rangle+i\left\langle A\left|\hat{\bar{\psi}}\left(x_{\bullet}, x_{\perp}\right) \overleftarrow{\hat{D}^{j}} \gamma_{j} \not p_{2} \gamma_{i} \hat{\psi}(0)\right| A\right\rangle\right]
\end{aligned}
$$

Using QCD equations of motion (3.1) we can rewrite the r.h.s. of eq. (4.32) as

$$
\begin{aligned}
& \int d x_{\bullet} d x_{\perp} e^{-i \alpha_{q} x_{\bullet}+i(k, x)_{\perp}}\left[k^{j}\left\langle A\left|\hat{\bar{\psi}}\left(x_{\bullet}, x_{\perp}\right) \gamma_{j} \not p_{2} \gamma_{i} \hat{\psi}(0)\right| A\right\rangle+\alpha_{q}\left\langle A\left|\hat{\bar{\psi}}\left(x_{\bullet}, x_{\perp}\right) \not p_{1} p_{2} \gamma_{i} \hat{\psi}(0)\right| A\right\rangle\right] \\
& =\int d x_{\bullet} d x_{\perp} e^{-i \alpha_{q} x_{\bullet}+i(k, x)_{\perp}}\left[-k_{i}\left\langle A\left|\hat{\bar{\psi}}\left(x_{\bullet}, x_{\perp}\right) \not p_{2} \hat{\psi}(0)\right| A\right\rangle+\alpha_{q} \frac{s}{2}\left\langle A\left|\hat{\bar{\psi}}\left(x_{\bullet}, x_{\perp}\right) \gamma_{i} \hat{\psi}(0)\right| A\right\rangle\right. \\
& \left.\quad+\frac{2 i}{s} \epsilon_{\bullet * i j} k^{j}\left\langle A\left|\hat{\bar{\psi}}\left(x_{\bullet}, x_{\perp}\right) \not p_{2} \gamma_{5} \hat{\psi}(0)\right| A\right\rangle-i \epsilon_{\bullet * i j}\left\langle A\left|\hat{\bar{\psi}}\left(x_{\bullet}, x_{\perp}\right) \gamma^{j} \gamma_{5} \hat{\psi}(0)\right| A\right\rangle\right] \\
& =-k_{i} 8 \pi^{3} s f_{1}\left(\alpha_{q}, k_{\perp}^{2}\right)+8 \pi^{3} s \alpha_{q} k_{i}\left[f_{\perp}\left(\alpha_{q}, k_{\perp}^{2}\right)+g^{\perp}\left(\alpha_{q}, k_{\perp}^{2}\right)\right],
\end{aligned}
$$

where we used parametrizations (4.13) and (4.17) for the leading power matrix elements.

Now, the second term in eq. (4.33) contains extra $\alpha_{q}$ with respect to the first term, so it should be neglected in our kinematical region $s \gg Q^{2} \gg q_{\perp}^{2}$ and we get

$$
\begin{aligned}
& \frac{g}{8 \pi^{3} s} \int d x_{\bullet} d x_{\perp} e^{-i \alpha_{q} x_{\bullet}+i(k, x)_{\perp}}\left\langle A\left|\hat{\bar{\psi}}^{f}\left(x_{\bullet}, x_{\perp}\right) p_{2}\left[\hat{A}_{i}\left(x_{\bullet}, x_{\perp}\right)-i \gamma_{5} \hat{\tilde{A}}_{i}\left(x_{\bullet}, x_{\perp}\right)\right] \hat{\psi}^{f}(0)\right| A\right\rangle \\
& =-k_{i} f_{1}^{f}\left(\alpha_{q}, k_{\perp}^{2}\right)+O\left(\alpha_{q}\right) .
\end{aligned}
$$

By complex conjugation

$$
\begin{aligned}
& \frac{g}{8 \pi^{3} s} \int d x_{\perp} d x_{\bullet} e^{-i \alpha_{q} x_{\bullet}+i(k, x) \perp}\left\langle A\left|\hat{\bar{\psi}}_{f}\left(x_{\bullet}, x_{\perp}\right) \not p_{2}\left[\hat{A}_{i}(0)+i \gamma_{5} \hat{\tilde{A}}_{i}(0)\right] \hat{\psi}_{f}(0)\right| A\right\rangle \\
& =-k_{i} f_{1 f}\left(\alpha_{q}, k_{\perp}^{2}\right) .
\end{aligned}
$$

For the corresponding antiquark distributions we get

$$
\begin{aligned}
\frac{g}{8 \pi^{3} s} \int & d x_{\perp} d x_{\bullet} e^{-i \alpha x_{\bullet}+i(k, x)_{\perp}}\left\langle A\left|\hat{\bar{\psi}}_{f}(0) \not p_{2}\left[\hat{A}_{i}\left(x_{\bullet}, x_{\perp}\right)+i \gamma_{5} \hat{\tilde{A}}_{i}\left(x_{\bullet}, x_{\perp}\right)\right] \hat{\psi}_{f}\left(x_{\bullet}, x_{\perp}\right)\right| A\right\rangle \\
= & \frac{1}{8 \pi^{3} s} \int d x_{\bullet} d x_{\perp} e^{-i \alpha_{q} x_{\bullet}+i(k, x) \perp}\left[-k_{j}\left\langle A\left|\hat{\bar{\psi}}(0) \gamma_{i} \not p_{2} \gamma^{j} \hat{\psi}\left(x_{\bullet}, x_{\perp}\right)\right| A\right\rangle\right. \\
& \left.-i\left\langle A\left|\hat{\bar{\psi}}(0) \gamma_{i} \not p_{2} \gamma^{j} \hat{D}_{j} \hat{\psi}\left(x_{\bullet}, x_{\perp}\right)\right| A\right\rangle\right]=-k_{i} \bar{f}_{1 f}\left(\alpha_{q}, k_{\perp}^{2}\right)
\end{aligned}
$$

and

$$
\begin{aligned}
& \frac{g}{8 \pi^{3} s} \int d x_{\perp} d x_{\bullet} e^{-i \alpha x_{\bullet}+i(k, x)_{\perp}}\left\langle A\left|\hat{\bar{\psi}}_{f}(0) \not p_{2}\left[\hat{A}_{i}(0)-i \gamma_{5} \hat{\tilde{A}}_{i}(0)\right] \hat{\psi}_{f}\left(x_{\bullet}, x_{\perp}\right)\right| A\right\rangle \\
& =-k_{i} \bar{f}_{1 f}\left(\alpha, k_{\perp}^{2}\right) .
\end{aligned}
$$


The corresponding target matrix elements are obtained by trivial replacements $x_{*} \leftrightarrow$ $x_{\bullet}, \alpha_{q} \leftrightarrow \beta_{q}$ and $\not p_{2} \leftrightarrow \not p_{1}$.

Next, let us consider

$$
\begin{aligned}
\frac{g}{8 \pi^{3} s} \int & d x_{\bullet} d x_{\perp} e^{-i \alpha_{q} x_{\bullet}+i(k, x)_{\perp}}\left\langle A\left|\hat{\psi}\left(x_{\bullet}, x_{\perp}\right) \not p_{2} \gamma^{i} \hat{A}_{i}\left(x_{\bullet}, x_{\perp}\right) \hat{\psi}(0)\right| A\right\rangle \\
= & \frac{1}{8 \pi^{3} s} \int d x_{\bullet} d x_{\perp} e^{-i \alpha_{q} x_{\bullet}+i(k, x)_{\perp}} \\
& \times\left[k_{i}\left\langle A\left|\hat{\psi}\left(x_{\bullet}, x_{\perp}\right) \gamma^{i} \not p_{2} \hat{\psi}(0)\right| A\right\rangle+i\left\langle A\left|\hat{\psi}\left(x_{\bullet}, x_{\perp}\right) \overleftarrow{\hat{D}}_{i} \gamma^{i} \not p_{2} \hat{\psi}(0)\right| A\right\rangle\right] .
\end{aligned}
$$

Using QCD equation of motion and parametrization (4.18), one can rewrite the r.h.s. of this equation as

$$
\begin{aligned}
& \frac{1}{8 \pi^{3} s} \int d x_{\bullet} d x_{\perp} e^{-i \alpha_{q} x_{\bullet}+i(k, x)_{\perp}}\left[k_{i}\left\langle A\left|\hat{\bar{\psi}}\left(x_{\bullet}, x_{\perp}\right) \gamma^{i} \not p_{2} \hat{\psi}(0)\right| A\right\rangle+\alpha_{q}\left\langle A\left|\hat{\bar{\psi}}\left(x_{\bullet}, x_{\perp}\right) \not p_{1} \not p_{2} \hat{\psi}(0)\right| A\right\rangle\right] \\
& \quad=i \frac{k_{\perp}^{2}}{m_{N}} h_{1}^{\perp}\left(\alpha_{q}, k_{\perp}^{2}\right)+\alpha_{q} m_{N}\left[e\left(\alpha, k_{\perp}^{2}\right)+i h\left(\alpha, k_{\perp}^{2}\right)\right] .
\end{aligned}
$$

Again, only the first term contributes in our kinematical region so we finally get

$$
\frac{g}{8 \pi^{3} s} \int d x_{\bullet} d x_{\perp} e^{-i \alpha_{q} x_{\bullet}+i(k, x)_{\perp}}\left\langle A\left|\hat{\bar{\psi}}^{f}\left(x_{\bullet}, x_{\perp}\right) p_{2} \gamma^{i} \hat{A}_{i}\left(x_{\bullet}, x_{\perp}\right) \hat{\psi}^{f}(0)\right| A\right\rangle=i \frac{k_{\perp}^{2}}{m_{N}} h_{1 f}^{\perp}\left(\alpha_{q}, k_{\perp}^{2}\right) \text {. }
$$

By complex conjugation we obtain

$$
\frac{g}{8 \pi^{3} s} \int d x_{\bullet} d x_{\perp} e^{-i \alpha_{q} x_{\bullet}+i(k, x)_{\perp}}\left\langle A\left|\hat{\bar{\psi}}^{f}\left(x_{\bullet}, x_{\perp}\right) \not p_{2} \gamma^{i} \hat{A}_{i}(0) \hat{\psi}^{f}(0)\right| A\right\rangle=i \frac{k_{\perp}^{2}}{m_{N}} h_{1 f}^{\perp}\left(\alpha_{q}, k_{\perp}^{2}\right) .
$$

For corresponding antiquark distributions one gets in a similar way

$$
\begin{array}{r}
\frac{g}{8 \pi^{3} s} \int d x_{\bullet} d x_{\perp} e^{-i \alpha_{q} x_{\bullet}+i(k, x)_{\perp}}\left\langle A\left|\hat{\bar{\psi}}^{f}(0) \not p_{2} \gamma^{i} \hat{A}_{i}\left(x_{\bullet}, x_{\perp}\right) \hat{\psi}^{f}\left(x_{\bullet}, x_{\perp}\right)\right| A\right\rangle=i \frac{k_{\perp}^{2}}{m_{N}} \bar{h}_{1 f}^{\perp}\left(\alpha_{q}, k_{\perp}^{2}\right), \\
\frac{g}{8 \pi^{3} s} \int d x_{\bullet} d x_{\perp} e^{-i \alpha_{q} x_{\bullet}+i(k, x)_{\perp}}\left\langle A\left|\hat{\bar{\psi}}^{f}(0) \not p_{2} \gamma^{i} \hat{A}_{i}(0) \hat{\psi}^{f}\left(x_{\bullet}, x_{\perp}\right)\right| A\right\rangle=i \frac{k_{\perp}^{2}}{m_{N}} \bar{h}_{1 f}^{\perp}\left(\alpha_{q}, k_{\perp}^{2}\right) .
\end{array}
$$

The target matrix elements are obtained by usual replacements $x_{*} \leftrightarrow x_{\bullet}, \alpha_{q} \leftrightarrow \beta_{q}$ and $\not p_{2} \leftrightarrow \not p_{1}$.

\subsubsection{Sixth line in eq. (4.19)}

In this section we consider $\left[\bar{\psi}_{A}(x) \breve{\gamma}_{\mu} \psi_{B}(x)\right]\left[\bar{\Xi}_{1 B}(0) \breve{\gamma}^{\mu} \Xi_{2 A}(0)\right]$ which turns to

$$
\begin{aligned}
& {\left[\bar{\psi}_{A}^{m}(x) \gamma_{\mu}\left(a-\gamma_{5}\right) \psi_{B}^{m}(x)\right]\left[\bar{\Xi}_{1 B}^{n}(0) \gamma^{\mu}\left(a-\gamma_{5}\right) \Xi_{2 A}^{n}(0)\right]} \\
& =\frac{1+a^{2}}{2}\left\{\left[\bar{\psi}_{A}^{m}(x) \gamma_{i} \Xi_{2 A}^{n}(0)\right]\left[\bar{\Xi}_{1 B}^{n}(0) \gamma^{i} \psi_{B}^{m}(x)\right]+\left(\gamma_{i} \otimes \gamma^{i} \leftrightarrow \gamma_{i} \gamma_{5} \otimes \gamma^{i} \gamma_{5}\right)\right\} \\
& \quad+\left(1-a^{2}\right)\left\{\left[\bar{\psi}_{A}^{m}(x) \Xi_{2 A}^{n}(0)\right]\left[\bar{\Xi}_{1 B}^{n}(0) \psi_{B}^{m}(x)\right]-\left(1 \otimes 1 \leftrightarrow \gamma_{5} \otimes \gamma_{5}\right)\right\} \\
& \quad-a\left\{\left[\bar{\psi}_{A}^{m}(x) \gamma_{i} \Xi_{2 A}^{n}(0)\right]\left[\bar{\Xi}_{1 B}^{n}(0) \gamma^{i} \gamma_{5} \psi_{B}^{m}(x)\right]+\left(\gamma_{i} \otimes \gamma^{i} \gamma_{5} \leftrightarrow \gamma_{i} \gamma_{5} \otimes \gamma^{i}\right)\right\}+O\left(\frac{m_{\perp}^{8}}{s}\right)
\end{aligned}
$$


after Fierz transformation (cf. eq. (4.8)). After separation of color singlet contributions

$$
\begin{aligned}
& \left\langle A, B\left|\left(\bar{\Psi}_{A}^{m}\left(A_{i}\right)^{l n} \psi_{A}^{k}\right)\left(\bar{\Psi}_{B}^{l}\left(B_{j}\right)^{n k} \psi_{B}^{m}\right)\right| A, B\right\rangle \\
& =\left\langle A, B\left|\left(\bar{\Psi}_{A}^{m}\left(A_{i}\right)^{n k} \psi_{A}^{k}\right)\left(\bar{\Psi}_{B}^{l}\left(B_{j}\right)^{l n} \psi_{B}^{m}\right)\right| A, B\right\rangle+i f^{a b c}\left\langle A, B\left|\left(\bar{\Psi}_{A}^{m}\left(t^{c}\right)^{l k} A_{i}^{a} \psi_{A}^{k}\right)\left(\bar{\Psi}_{B}^{l} B_{j}^{b} \psi_{B}^{m}\right)\right| A, B\right\rangle \\
& =\frac{1}{N_{c}}\left\langle A\left|\bar{\Psi}_{A} A_{i} \psi_{A}\right| A\right\rangle\left\langle B\left|\bar{\Psi}_{B} B_{j} \psi_{B}\right| B\right\rangle+2 i f^{a b c}\left\langle A\left|\bar{\Psi}_{A} t^{d} t^{c} A_{i}^{a} \psi_{A}\right| A\right\rangle\left\langle B\left|\bar{\Psi}_{B} t^{d} B_{j}^{b} \psi_{B}\right| B\right\rangle \\
& =-\frac{1}{N_{c}\left(N_{c}^{2}-1\right)}\left\langle A\left|\bar{\Psi}_{A} A_{i} \psi_{A}\right| A\right\rangle\left\langle B\left|\bar{\Psi}_{B} B_{j} \psi_{B}\right| B\right\rangle
\end{aligned}
$$

we obtain

$$
\begin{aligned}
&-g^{-2} N_{c}\left(N_{c}^{2}-1\right)\left[\bar{\psi}_{A}(x) \breve{\gamma}_{\mu} \psi_{B}(x)\right] {\left[\bar{\Xi}_{1 B}(0) \breve{\gamma}^{\mu} \Xi_{2 A}(0)\right] } \\
&=\frac{1+a^{2}}{2 s^{2}}\left\{\left[\bar{\psi}_{A}(x) \gamma_{i} \not p_{2} \gamma^{j} A_{k}(0) \frac{1}{\alpha} \psi_{A}(0)\right]\left[\left(\bar{\psi}_{B} \frac{1}{\beta}\right)(0) \gamma^{k} \not p_{1} \gamma^{i} B_{j}(0) \psi_{B}(x)\right]\right. \\
&\left.+\left(\gamma_{i} \otimes \gamma^{i} \leftrightarrow \gamma_{i} \gamma_{5} \otimes \gamma^{i} \gamma_{5}\right)\right\} \\
&+\frac{a^{2}-1}{s^{2}}\left\{\left[\bar{\psi}_{A}(x) \not p_{2} \gamma^{j} A_{k}(0) \frac{1}{\alpha} \psi_{A}(0)\right]\left[\left(\bar{\psi}_{B} \frac{1}{\beta}\right)(0) \not p_{1} \gamma^{k} B_{j}(0) \psi_{B}(x)\right]\right. \\
&\left.-\left(\gamma^{j} \otimes \gamma^{k} \leftrightarrow \gamma^{j} \gamma_{5} \otimes \gamma^{k} \gamma_{5}\right)\right\} \\
&-\frac{a}{s^{2}}\left\{\left[\bar{\psi}_{A}(x) \gamma_{i} \not p_{2} \gamma^{j} A_{k}(0) \frac{1}{\alpha} \psi_{A}(0)\right]\left[\left(\bar{\psi}_{B} \frac{1}{\beta}\right)(0) \gamma^{k} \not p_{1} \gamma^{i} \gamma_{5} B_{j}(0) \psi_{B}(x)\right]\right. \\
&\left.+\left(\gamma_{i} \otimes \gamma^{i} \gamma_{5} \leftrightarrow \gamma_{i} \gamma_{5} \otimes \gamma^{i}\right)\right\}+O\left(\frac{m_{\perp}^{8}}{s}\right),
\end{aligned}
$$

which can be rewritten as

$$
\begin{aligned}
& -g^{-2} N_{c}\left(N_{c}^{2}-1\right)\left[\bar{\psi}_{A}(x) \breve{\gamma}_{\mu} \psi_{B}(x)\right]\left[\bar{\Xi}_{1 B}(0) \breve{\gamma}^{\mu} \Xi_{2 A}(0)\right]=\frac{a^{2}-1}{s^{2}}\left[\bar{\psi}_{A}(x) A_{k}(0) \not p_{2} \gamma_{j} \frac{1}{\alpha} \psi_{A}(0)\right] \\
& {\left[\left(\bar{\psi}_{B} \frac{1}{\beta}\right)(0)\left(B^{j}(0) \not p_{1} \gamma^{k}-j \leftrightarrow k+g^{j k} B^{i}(0) \not p_{1} \gamma_{i}\right) \psi_{B}(x)\right]+O\left(\frac{m_{\perp}^{8}}{s}\right),}
\end{aligned}
$$

where we again used formulas (B.3), (B.5), and (B.8) from appendix B.

Next, it is easy to see that $\frac{1}{\alpha}$ and $\frac{1}{\beta}$ in eq. (4.45) give $\frac{1}{\alpha_{q}}$ and $-\frac{1}{\beta_{q}}$ :

$$
\begin{aligned}
& \int d x_{\bullet} e^{-i \alpha_{q} x_{\bullet}}\left\langle A\left|\hat{\bar{\psi}}\left(x_{\bullet}, x_{\perp}\right) \Gamma \hat{A}_{i}(0) \frac{1}{\alpha} \hat{\psi}(0)\right| A\right\rangle \\
& =\frac{1}{\alpha_{q}} \int d x_{\bullet} e^{-i \alpha_{q} x_{\bullet}}\left\langle A\left|\hat{\bar{\psi}}\left(x_{\bullet}, x_{\perp}\right) \Gamma\left[\hat{A}_{i}(0) \hat{\psi}(0)+\frac{2}{s} \hat{F}_{* i}(0) \int_{-\infty}^{0} d x_{\bullet}^{\prime} \hat{\psi}\left(x_{\bullet}^{\prime}, 0_{\perp}\right)\right]\right| A\right\rangle, \\
& \int d x_{*} e^{-i \beta_{q} x_{*}}\left\langle B\left|\left(\hat{\bar{\psi}} \frac{1}{\beta}\right)(0) \hat{A}_{i}(0) \Gamma \hat{\psi}\left(x_{*}, x_{\perp}\right)\right| B\right\rangle \\
& =-\frac{1}{\beta_{q}} \int d x_{*} e^{-i \beta_{q} x_{*}}\left\langle B\left|\left[\hat{\bar{\psi}}(0) \hat{A}_{i}(0)+\int_{-\infty}^{0} d x_{*}^{\prime} \hat{\bar{\psi}}\left(x_{*}^{\prime}, 0_{\perp}\right) \frac{2}{s} \hat{F}_{\bullet i}(0)\right] \Gamma \hat{\psi}\left(x_{*}, x_{\perp}\right)\right| B\right\rangle,
\end{aligned}
$$

where $\Gamma$ is any of the Dirac matrices in eq. (4.45). 
The corresponding contribution to $W\left(\alpha_{q}, \beta_{q}, x_{\perp}\right)$ takes the form

$$
\begin{aligned}
& \frac{e^{2} g^{2}}{8(2 \pi)^{4} s_{W}^{2} c_{W}^{2} N_{c}\left(N_{c}^{2}-1\right) Q^{2}} \int d x_{\bullet} d x_{*} e^{-i \alpha_{q} x_{\bullet}-i \beta_{q} x_{*}} \\
& \times \frac{a^{2}-1}{s^{2}}\left\langle A\left|\hat{\bar{\psi}}\left(x_{\bullet}, x_{\perp}\right) \not p_{2} \gamma^{j}\left[\hat{A}_{j}(0) \hat{\psi}(0)+\frac{2}{s} \hat{F}_{* j}(0) \int_{-\infty}^{0} d x_{\bullet}^{\prime} \hat{\psi}\left(x_{\bullet}^{\prime}, 0_{\perp}\right)\right]\right| A\right\rangle \\
& \quad \times\left\langle B\left|\left[\hat{\bar{\psi}}(0) \hat{A}_{k}(0)+\int_{-\infty}^{0} d x_{*}^{\prime} \hat{\bar{\psi}}\left(x_{*}^{\prime}, 0_{\perp}\right) \frac{2}{s} \hat{F}_{\bullet}(0)\right] \not p_{1} \gamma^{k} \hat{\psi}(x)\right| B\right\rangle\left(1+O\left(\frac{m_{\perp}^{2}}{s}\right)\right),
\end{aligned}
$$

where we have used the fact that

$$
\left\langle B\left|\left\{\left[\hat{\bar{\psi}}(0) \hat{A}_{j}(0)+\int_{-\infty}^{0} d x_{*}^{\prime} \hat{\bar{\psi}}\left(x_{*}^{\prime}, 0_{\perp}\right) \frac{2}{s} \hat{F}_{\bullet j}(0)\right] \not p_{1} \gamma_{k}-j \leftrightarrow k\right\} \hat{\psi}\left(x_{*}, x_{\perp}\right)\right| B\right\rangle=0
$$

for the unpolarized hadron.

Similarly,

$$
\begin{aligned}
& -g^{-2} N_{c}\left(N_{c}^{2}-1\right)\left[\bar{\Xi}_{2 A}(x) \breve{\gamma}_{\mu} \Xi_{1 B}(x)\right]\left[\bar{\psi}_{B}(0) \breve{\gamma}^{\mu} \psi_{A}(0)\right]=\frac{a^{2}-1}{s^{2}}\left[\left(\bar{\psi}_{A} \frac{1}{\alpha}\right)(x) A_{k}(x) \not p_{2} \gamma_{j} \psi_{A}(0)\right] \\
& \times\left[\bar{\psi}_{B}(0)\left(B^{j}(x) \not p_{1} \gamma^{k}-j \leftrightarrow k+g^{j k} B^{i}(x) \not p_{1} \gamma_{i}\right) \frac{1}{\beta} \psi_{B}(x)\right]+O\left(\frac{m_{\perp}^{8}}{s}\right),
\end{aligned}
$$

so the corresponding contribution to $W\left(\alpha_{q}, \beta_{q}, x_{\perp}\right)$ is

$$
\begin{aligned}
& \frac{g^{2} e^{2}}{8(2 \pi)^{4} s_{W}^{2} c_{W}^{2} N_{c}\left(N_{c}^{2}-1\right) Q^{2}} \frac{a^{2}-1}{s^{2}} \int d x_{\bullet} d x_{*} e^{-i \alpha_{q} x_{\bullet}-i \beta_{q} x_{*}} \\
& \times\left\langle A\left|\left[\hat{\bar{\psi}}\left(x_{\bullet}, x_{\perp}\right) \hat{A}_{j}\left(x_{\bullet}, x_{\perp}\right)+\int_{-\infty}^{x_{\bullet}} d x_{\bullet}^{\prime} \hat{\bar{\psi}}\left(x_{\bullet}^{\prime}, x_{\perp}\right) \frac{2}{s} \hat{F}_{* j}\left(x_{\bullet}, x_{\perp}\right)\right] \not p_{2} \gamma^{j} \hat{\psi}(0)\right| A\right\rangle \\
& \times\left\langle B\left|\hat{\bar{\psi}}(0) \not p_{1} \gamma^{k}\left[\hat{A}_{k}\left(x_{*}, x_{\perp}\right) \hat{\psi}\left(x_{*}, x_{\perp}\right)+\int_{-\infty}^{x_{*}} d x_{*}^{\prime} \frac{2}{s} \hat{F}_{\bullet k}\left(x_{*}, x_{\perp}\right) \hat{\psi}\left(x_{*}^{\prime}, x_{\perp}\right)\right]\right| B\right\rangle\left(1+O\left(\frac{m_{\perp}^{2}}{s}\right)\right) .
\end{aligned}
$$

Using parametrizations (4.52) and (4.53) from appendix 4.3.4 we obtain the contribution of the 6th line in eq. (4.19) in the form

$$
\begin{aligned}
& W^{6 \mathrm{th}}\left(\alpha_{q}, \beta_{q}, q_{\perp}\right)=-\frac{e^{2}}{4 s_{W}^{2} c_{W}^{2} N_{c}\left(N_{c}^{2}-1\right) Q^{2}} \int d^{2} k_{\perp} k_{\perp}^{2}(q-k)_{\perp}^{2} \\
& \quad \times\left[\left\{\frac{1}{m_{N}^{2}}\left(a_{u}^{2}-1\right)\left[h_{u}^{\mathrm{tw} 3}\left(\alpha_{q}, k_{\perp}\right) \bar{h}_{u}^{\mathrm{tw} 3}\left(\beta_{q}, q_{\perp}-k_{\perp}\right)+\tilde{h}_{u}^{\mathrm{tw} 3}\left(\alpha_{q}, k_{\perp}\right) \tilde{\bar{h}}_{u}^{\mathrm{tw} 3}\left(\beta_{q}, q_{\perp}-k_{\perp}\right)\right]\right.\right. \\
& \left.\left.\quad+\left(\alpha_{q} \leftrightarrow \beta_{q}\right)\right\}+\{u \leftrightarrow c\}+\{u \leftrightarrow d\}+\{u \leftrightarrow s\}\right]\left(1+O\left(\frac{m_{\perp}^{2}}{s}\right)\right)
\end{aligned}
$$

where quark $\leftrightarrow$ antiquark $\left(\alpha_{q} \leftrightarrow \beta_{q}\right)$ term comes from $x \leftrightarrow 0$ replacement, cf. eq. (4.31). 


\subsubsection{Parametrization of matrix elements from section 4.3.3}

In this section we present parametrization of matrix elements from section 4.3.3. Similarly to eqs. (4.40)-(4.42) we define

$$
\begin{aligned}
& \frac{g}{8 \pi^{3} s} \int d x_{\perp} d x_{\bullet} e^{-i \alpha x \bullet+i(k, x)_{\perp}}\langle A| \hat{\bar{\psi}}_{f}\left(x_{\bullet}, x_{\perp}\right) \not p_{2} \gamma^{i}\left\{\hat{A}_{i}(0) \hat{\psi}_{f}(0)\right. \\
& \left.\quad+\frac{2}{s} \hat{F}_{* i}(0) \int_{-\infty}^{0} d x_{\bullet}^{\prime} \hat{\psi}_{f}\left(x_{\bullet}^{\prime}, 0_{\perp}\right)\right\}|A\rangle=i \frac{k_{\perp}^{2}}{m_{N}}\left[h_{f}^{\mathrm{tw} 3}\left(\alpha, k_{\perp}^{2}\right)+i \tilde{h}_{f}^{\mathrm{tw} 3}\left(\alpha, k_{\perp}^{2}\right)\right] \\
& \frac{g}{8 \pi^{3} s} \int d x_{\perp} d x_{\bullet} e^{-i \alpha x \bullet+i(k, x)_{\perp}}\langle A| \hat{\bar{\psi}}_{f}(0) \not p_{2} \gamma^{i}\left\{\hat{A}_{i}\left(x_{\bullet}, x_{\perp}\right) \hat{\psi}_{f}\left(x_{\bullet}, x_{\perp}\right)\right. \\
& \left.\quad+\frac{2}{s} \hat{F}_{* i}\left(x_{\bullet}, x_{\perp}\right) \int_{-\infty}^{x} d x_{\bullet}^{\prime} \hat{\psi}_{f}\left(x_{\bullet}^{\prime}, x_{\perp}\right)\right\}|A\rangle=i \frac{k_{\perp}^{2}}{m_{N}}\left[\bar{h}_{f}^{\mathrm{tw} 3}\left(\alpha, k_{\perp}^{2}\right)+i \tilde{\bar{h}}_{f}^{\mathrm{tw} 3}\left(\alpha, k_{\perp}^{2}\right)\right]
\end{aligned}
$$

and similarly for the target matrix elements. Note that unlike two-quark matrix elements, quark-quark-gluon ones may have imaginary parts which we denote by functions with tildes.

By complex conjugation we get

$$
\begin{aligned}
& \frac{g}{8 \pi^{3} s} \int d x_{\perp} d x_{\bullet} e^{-i \alpha x_{\bullet}+i(k, x)_{\perp}}\langle A|\left\{\hat{\bar{\psi}}_{f}\left(x_{\bullet}, x_{\perp}\right) \hat{A}_{i}\left(x_{\bullet}, x_{\perp}\right)\right. \\
& \left.+\int_{-\infty}^{x} d x_{\bullet}^{\prime} \hat{\bar{\psi}}_{f}\left(x_{\bullet}^{\prime}, x_{\perp}\right) \frac{2}{s} \hat{F}_{* i}\left(x_{\bullet}, x_{\perp}\right)\right\} \not p_{2} \gamma^{i} \hat{\psi}_{f}(0)|A\rangle=i \frac{k_{\perp}^{2}}{m_{N}}\left[h_{f}^{\mathrm{tw} 3}\left(\alpha, k_{\perp}^{2}\right)-i \tilde{h}_{f}^{\mathrm{tw} 3}\left(\alpha, k_{\perp}^{2}\right)\right], \\
& \frac{g}{8 \pi^{3} s} \int d x_{\perp} d x_{\bullet} e^{-i \alpha x_{\bullet}+i(k, x)_{\perp}}\langle A|\left\{\hat{\bar{\psi}}_{f}(0) \hat{A}_{i}(0)\right. \\
& \left.\quad+\int_{-\infty}^{0} d x_{\bullet}^{\prime} \hat{\bar{\psi}}_{f}\left(x_{\bullet}^{\prime}, 0_{\perp}\right) \frac{2}{s} \hat{F}_{* i}(0)\right\} \not p_{2} \gamma^{i} \hat{\psi}_{f}\left(x_{\bullet}, x_{\perp}\right)|A\rangle=i \frac{k_{\perp}^{2}}{m_{N}}\left[\bar{h}_{f}^{\mathrm{tw} 3}\left(\alpha, k_{\perp}^{2}\right)-i \tilde{\bar{h}}_{f}^{\mathrm{tw} 3}\left(\alpha, k_{\perp}^{2}\right)\right]
\end{aligned}
$$

and similarly for the target matrix elements.

For completeness, let us present the structure of gauge links in an arbitrary gauge, for example:

$$
\begin{aligned}
& \left\langle A\left|\left\{\hat{\bar{\psi}}_{f}\left(x_{\bullet}, x_{\perp}\right) \hat{A}_{j}\left(x_{\bullet}, x_{\perp}\right)+\int_{-\infty}^{x_{\bullet}} d x_{\bullet}^{\prime} \hat{\bar{\psi}}_{f}\left(x_{\bullet}^{\prime}, x_{\perp}\right) \frac{2}{s} \hat{F}_{* j}\left(x_{\bullet}, x_{\perp}\right)\right\} \not p_{2} \gamma_{i} \hat{\psi}_{f}(0)\right| A\right\rangle \\
& \rightarrow \frac{2}{s} \int_{-\infty}^{x_{\bullet}} d x_{\bullet}^{\prime}\langle A|\left\{\hat{\bar{\psi}}_{f}\left(x_{\bullet}, x_{\perp}\right)\left[x_{\bullet}, x_{\bullet}^{\prime}\right]_{x} \hat{F}_{* j}\left(x_{\bullet}^{\prime}, x_{\perp}\right)\left[x_{\bullet}^{\prime},-\infty\right]_{x}\right. \\
& \left.\quad+\hat{\bar{\psi}}_{f}\left(x_{\bullet}^{\prime}, x_{\perp}\right)\left[x_{\bullet}^{\prime}, x_{\bullet}\right]_{x} \hat{F}_{* j}\left(x_{\bullet}, x_{\perp}\right)\left[x_{\bullet},-\infty\right]_{x}\right\}\left[x_{\perp}, 0_{\perp}\right]_{-\infty}\left[-\infty_{\bullet}, 0_{\bullet}\right]_{0_{\perp}} \not p_{2} \gamma_{i} \hat{\psi}_{f}(0)|A\rangle .
\end{aligned}
$$

\subsection{Power corrections from $\mathcal{J}_{A}^{\mu}(x) \mathcal{J}_{B \mu}(0)$ terms}

Power corrections of the second type come from the terms

$$
\begin{aligned}
& \bar{\Psi}_{A}(x) \breve{\gamma}^{\mu} \Psi_{A}(x) \bar{\Psi}_{B}(0) \breve{\gamma}_{\mu} \Psi_{B}(0)+x \leftrightarrow 0=\bar{\Psi}_{A}^{(0)}(x) \breve{\gamma}^{\mu} \Psi_{A}^{(0)}(x) \bar{\Psi}_{B}^{(0)}(0) \breve{\gamma}_{\mu} \Psi_{B}^{(0)}(0) \\
& \quad+\bar{\Psi}_{A}^{(1)}(x) \breve{\gamma}^{\mu} \Psi_{A}^{(0)}(x) \bar{\Psi}_{B}^{(0)}(0) \breve{\gamma}_{\mu} \Psi_{B}^{(0)}(0)+\bar{\Psi}_{A}^{(0)}(x) \breve{\gamma}^{\mu} \Psi_{A}^{(1)}(x) \bar{\Psi}_{B}^{(0)}(0) \breve{\gamma}_{\mu} \Psi_{B}^{(0)}(0) \\
& \quad+\bar{\Psi}_{A}^{(0)}(x) \breve{\gamma}^{\mu} \Psi_{A}^{(0)}(x) \bar{\Psi}_{B}^{(1)}(0) \breve{\gamma}_{\mu} \Psi_{B}^{(0)}(0)+\bar{\Psi}_{A}^{(0)}(x) \breve{\gamma}^{\mu} \Psi_{A}^{(0)}(x) \bar{\Psi}_{B}^{(0)}(0) \breve{\gamma}_{\mu} \Psi_{B}^{(1)}(0)+x \leftrightarrow 0+\ldots
\end{aligned}
$$


In appendix C.4, we will demonstrate that terms $\sim \Psi^{(1)}$ are small in our kinematical region $s \gg Q^{2} \gg q_{\perp}^{2}$.

Terms $\sim \Psi^{(0)}$ read

$$
\begin{aligned}
{[} & \left.\left(\bar{\psi}_{A}+\bar{\Xi}_{2 A}\right)(x) \breve{\gamma}_{\mu}\left(\psi_{A}+\Xi_{2 A}\right)(x)\right]\left[\left(\bar{\psi}_{B}+\bar{\Xi}_{1 B}\right)(0) \breve{\gamma}^{\mu}\left(\psi_{B}+\Xi_{1 B}\right)(0)\right]+x \leftrightarrow 0 \\
= & {\left[\bar{\psi}_{A}(x) \breve{\gamma}_{\mu} \psi_{A}(x)\right]\left[\bar{\psi}_{B}(0) \breve{\gamma}^{\mu} \psi_{B}(0)\right] } \\
& +\left[\bar{\Xi}_{2 A}(x) \breve{\gamma}_{\mu} \psi_{A}(x)\right]\left[\bar{\psi}_{B}(0) \breve{\gamma}^{\mu} \psi_{B}(0)\right]+\left[\bar{\psi}_{A}(x) \breve{\gamma}_{\mu} \Xi_{2 A}(x)\right]\left[\bar{\psi}_{B}(0) \breve{\gamma}^{\mu} \psi_{B}(0)\right] \\
& +\left[\bar{\psi}_{A}(x) \breve{\gamma}_{\mu} \psi_{A}(x)\right]\left[\bar{\Xi}_{1 B}(0) \breve{\gamma}^{\mu} \psi_{B}(0)\right]+\left[\bar{\psi}_{A}(x) \breve{\gamma}_{\mu} \psi_{A}(x)\right]\left[\bar{\psi}_{B}(0) \breve{\gamma}^{\mu} \Xi_{1 B}(0)\right] \\
& +\left[\bar{\Xi}_{2 A}(x) \breve{\gamma}_{\mu} \Xi_{2 A}(x)\right]\left[\bar{\psi}_{B}(0) \breve{\gamma}^{\mu} \psi_{B}(0)\right]+\left[\bar{\psi}_{A}(x) \breve{\gamma}_{\mu} \psi_{A}(x)\right]\left[\bar{\Xi}_{1 B}(0) \breve{\gamma}^{\mu} \Xi_{1 B}(0)\right] \\
& +\left[\bar{\Xi}_{2 A}(x) \breve{\gamma}_{\mu} \psi_{A}(x)\right]\left[\bar{\psi}_{B}(0) \breve{\gamma}^{\mu} \Xi_{1 B}(0)\right]+\left[\bar{\psi}_{A}(x) \breve{\gamma}_{\mu} \Xi_{2 A}(x)\right]\left[\bar{\Xi}_{1 B}(0) \breve{\gamma}^{\mu} \psi_{B}(0)\right] \\
& +\left[\bar{\Xi}_{2 A}(x) \breve{\gamma}_{\mu} \psi_{A}(x)\right]\left[\bar{\Xi}_{1 B}(0) \breve{\gamma}^{\mu} \psi_{B}(0)\right]+\left[\bar{\psi}_{A}(x) \breve{\gamma}_{\mu} \Xi_{2 A}(x)\right]\left[\bar{\psi}_{B}(0) \breve{\gamma}^{\mu} \Xi_{1 B}(0)\right]+x \leftrightarrow 0 .
\end{aligned}
$$

As we prove in appendix C.2, the leading power correction comes from last two lines in eq. (4.56). We will consider them in turn.

\subsubsection{Last two lines in eq. (4.56)}

Using eq. (3.27) and separating color-singlet matrix elements, we rewrite the sixth line in eq. (4.56) as

$$
\begin{aligned}
& {\left[\bar{\Xi}_{2 A}(x) \breve{\gamma}_{\mu} \psi_{A}(x)\right]\left[\bar{\psi}_{B}(0) \breve{\gamma}^{\mu} \Xi_{1 B}(0)\right]+\left[\bar{\psi}_{A}(x) \breve{\gamma}_{\mu} \Xi_{2 A}(x)\right]\left[\bar{\Xi}_{1 B}(0) \breve{\gamma}^{\mu} \psi_{A}(0)\right] } \\
&=\frac{g^{2}}{\left(N_{c}^{2}-1\right) s^{2}}\left(\left[\left(\bar{\psi}_{A} \frac{1}{\alpha}\right)(x) \gamma^{j} \not p_{2} \breve{\gamma}_{\mu} A_{k}(0) \psi_{A}(x)\right]\left[\bar{\psi}_{B}(0) \breve{\gamma}^{\mu} \not \not_{1} \gamma^{k} B_{j}(x) \frac{1}{\beta} \psi_{B}(0)\right]\right. \\
&\left.+\left[\bar{\psi}_{A}(x) \breve{\gamma}^{\mu} \not p_{2} \gamma^{j} A_{k}(0) \frac{1}{\alpha} \psi_{A}(x)\right]\left[\left(\bar{\psi}_{B} \frac{1}{\beta}\right)(0) \gamma^{k} \not \not_{1} \breve{\gamma}_{\mu} B_{j}(x) \psi_{B}(0)\right]\right)+x \leftrightarrow 0 \\
&= \frac{g^{2}\left(a^{2}-1\right)}{\left(N_{c}^{2}-1\right) s^{2}}\left(\left[\left(\bar{\psi}_{A} \frac{1}{\alpha}\right)(x) \not \not_{2}\left(A_{i}+i \gamma_{5} \tilde{A}_{i}\right)(0) \psi_{A}(x)\right]\left[\bar{\psi}_{B}(0) \not p_{1}\left(B^{i}(x)-i \gamma_{5} \tilde{B}^{i}\right)(x) \frac{1}{\beta} \psi_{B}(0)\right]\right. \\
&\left.+\left[\bar{\psi}_{A}(x) \not p_{2}\left[A_{i}(0)-i \gamma_{5} \tilde{A}_{i}(0)\right] \frac{1}{\alpha} \psi_{A}(x)\right]\left[\left(\bar{\psi}_{B} \frac{1}{\beta}\right)(0) \not p_{1}\left[B^{i}(x)+i \gamma_{5} \tilde{B}^{i}(x)\right] \psi_{B}(0)\right]\right)+x \leftrightarrow 0,
\end{aligned}
$$

where we used eqs. (B.4) and (B.5). For the forward matrix elements

$$
\begin{aligned}
\int d x_{\bullet} & e^{-i \alpha_{q} x_{\bullet}}\left\langle A\left|\left(\hat{\bar{\psi}} \frac{1}{\alpha}\right)\left(x_{\bullet}, x_{\perp}\right) \not p_{2} \hat{A}_{i}(0) \hat{\psi}\left(x_{\bullet}, x_{\perp}\right)\right| A\right\rangle \\
= & -\frac{1}{\alpha_{q}} \int d x_{\bullet} e^{-i \alpha_{q} x_{\bullet}} \int_{-\infty}^{x_{\bullet}} d x_{\bullet}^{\prime}\left\langle A\left|\hat{\bar{\psi}}\left(x_{\bullet}^{\prime}, x_{\perp}\right) \frac{2 \not p_{2}}{s} \hat{F}_{* i}(0) \hat{\psi}\left(x_{\bullet}, x_{\perp}\right)\right| A\right\rangle, \\
\int d x_{\bullet} & e^{-i \alpha_{q} x_{\bullet}}\left\langle A\left|\hat{\bar{\psi}}\left(x_{\bullet}, x_{\perp}\right) \not p_{2} \hat{A}_{i}(0) \frac{1}{\alpha} \hat{\psi}\left(x_{\bullet}, x_{\perp}\right)\right| A\right\rangle \\
= & \frac{1}{\alpha_{q}} \int d x \bullet e^{-i \alpha_{q} x_{\bullet}} \int_{-\infty}^{x_{\bullet}} d x_{\bullet}^{\prime}\left\langle A\left|\hat{\bar{\psi}}\left(x_{\bullet}, x_{\perp}\right) \frac{2 \not p_{2}}{s} \hat{F}_{* i}(0) \hat{\psi}\left(x_{\bullet}^{\prime}, x_{\perp}\right)\right| A\right\rangle, \\
\int d x_{*} & e^{-i \beta_{q} x_{*}}\left\langle B\left|\hat{\bar{\psi}}(0) \not p_{1} \hat{A}_{i}\left(x_{*}, x_{\perp}\right) \frac{1}{\beta} \hat{\psi}(0)\right| B\right\rangle \\
= & -\frac{1}{\beta_{q}} \int d x_{*} e^{-i \beta_{q} x_{*}} \int_{-\infty}^{0} d x_{*}^{\prime}\left\langle B\left|\hat{\bar{\psi}}(0) \frac{2 \not p_{1}}{s} \hat{F}_{\bullet i}\left(x_{*}, x_{\perp}\right) \hat{\psi}\left(x_{*}^{\prime}, 0_{\perp}\right)\right| B\right\rangle, \\
\int d x_{*} & e^{-i \beta_{q} x_{*}}\left\langle B\left|\left(\hat{\bar{\psi}} \frac{1}{\beta}\right)(0) \not p_{1} \hat{A}_{i}\left(x_{*}, x_{\perp}\right) \hat{\psi}(0)\right| B\right\rangle \\
= & \frac{1}{\beta_{q}} \int d x_{*} e^{-i \beta_{q} x_{*}} \int_{-\infty}^{0} d x_{*}^{\prime}\left\langle B\left|\hat{\bar{\psi}}\left(x_{*}^{\prime}, 0_{\perp}\right) \frac{2 \not p_{1}}{s} \hat{F}_{\bullet i}\left(x_{*}, x_{\perp}\right) \hat{\psi}(0)\right| B\right\rangle .
\end{aligned}
$$


The corresponding contribution to $W\left(\alpha_{q}, \beta_{q}, x_{\perp}\right)$ takes the form

$$
\begin{aligned}
& \frac{g^{2} e^{2}\left(a^{2}-1\right)}{8(2 \pi)^{4} s_{W}^{2} c_{W}^{2}\left(N_{c}^{2}-1\right) Q^{2} s^{2}} \int d x_{\bullet} d x_{*} e^{-i \alpha_{q} x_{\bullet}-i \beta_{q} x_{*}} \\
& \times\left\{\int _ { - \infty } ^ { x _ { \bullet } } d x _ { \bullet } ^ { \prime } \int _ { - \infty } ^ { 0 } d x _ { * } ^ { \prime } \left[\left\langle A\left|\hat{\bar{\psi}}\left(x_{\bullet}^{\prime}, x_{\perp}\right) \frac{2 \not p_{2}}{s}\left[\hat{F}_{* i}(0)+i \gamma_{5} \hat{\tilde{F}}_{* i}(0)\right] \hat{\psi}\left(x_{\bullet}, x_{\perp}\right)\right| A\right\rangle\right.\right. \\
& \times\left\langle B\left|\hat{\bar{\psi}}(0) \frac{2 \not p_{1}}{s}\left[\hat{F}_{\bullet}{ }^{i}\left(x_{*}, x_{\perp}\right)-i \gamma_{5} \hat{\tilde{F}}_{\bullet}{ }^{i}\left(x_{*}, x_{\perp}\right)\right] \hat{\psi}\left(x_{*}^{\prime}, 0_{\perp}\right)\right| B\right\rangle \\
&+\left\langle A\left|\hat{\bar{\psi}}\left(x_{\bullet}, x_{\perp}\right) \frac{2 \not p_{2}}{s}\left[\hat{F}_{* i}(0)-i \gamma_{5} \hat{\tilde{F}}_{* i}(0)\right] \hat{\psi}\left(x_{\bullet}^{\prime}, x_{\perp}\right)\right| A\right\rangle \\
&\left.\left.\times\left\langle B\left|\hat{\bar{\psi}}\left(x_{*}^{\prime}, 0_{\perp}\right) \frac{2 \not p_{1}}{s}\left[\hat{F}_{\bullet}^{i}\left(x_{*}, x_{\perp}\right)+i \gamma_{5} \hat{\tilde{F}}_{\bullet}^{i}\left(x_{*}, x_{\perp}\right)\right] \hat{\psi}(0)\right| B\right\rangle\right]+x \leftrightarrow 0\right\}\left(1+O\left(\frac{m_{\perp}^{2}}{s}\right)\right) .
\end{aligned}
$$

Similarly, for the seventh line in eq. (4.56) using eqs. (3.27) and (B.6) one obtains

$$
\begin{aligned}
{[} & \left.\bar{\Xi}_{2 A}(x) \breve{\gamma}_{\mu} \psi_{A}(x)\right]\left[\bar{\Xi}_{1 B}(0) \breve{\gamma}^{\mu} \psi_{B}(0)\right]+\left[\bar{\psi}_{A}(x) \breve{\gamma}_{\mu} \Xi_{2 A}(x)\right]\left[\bar{\psi}_{B}(0) \breve{\gamma}^{\mu} \Xi_{1 B}(0)\right]+x \leftrightarrow 0 \\
= & \frac{g^{2}}{\left(N_{c}^{2}-1\right) s^{2}}\left(\left[\left(\bar{\psi}_{A} \frac{1}{\alpha}\right)(x) \gamma^{j} \not p_{2} \breve{\gamma}_{\mu} A_{k}(0) \psi_{A}(x)\right]\left[\left(\bar{\psi}_{B} \frac{1}{\beta}\right)(0) \gamma^{k} \not p_{1} \breve{\gamma}^{\mu} B_{j}(x) \psi_{B}(0)\right]\right. \\
& \left.+\left[\bar{\psi}_{A}(x) \breve{\gamma}_{\mu} \not p_{2} \gamma^{j} A_{k}(0)\left(\frac{1}{\alpha} \psi_{A}\right)(x)\right]\left[\bar{\psi}_{B}(0) \breve{\gamma}^{\mu} \not p_{1} \gamma^{k} B_{j}(x)\left(\frac{1}{\beta} \psi_{B}\right)(0)\right]\right)+x \leftrightarrow 0 \\
= & \frac{g^{2}}{\left(N_{c}^{2}-1\right) s^{2}}\left(( 1 + a ^ { 2 } ) \left\{\left[\left(\bar{\psi}_{A} \frac{1}{\alpha}\right)(x) \not p_{2}\left[A_{i}(0)+i \gamma_{5} \tilde{A}_{i}(0)\right] \psi_{A}(x)\right]\right.\right. \\
& \times\left[\left(\bar{\psi}_{B} \frac{1}{\beta}\right)(0) \not p_{1}\left[B^{i}(x)+i \gamma_{5} \tilde{B}^{i}(x)\right] \psi_{B}(0)\right] \\
& \left.+\left[\bar{\psi}_{A}(x) \not p_{2}\left[A_{i}(0)-i \gamma_{5} \tilde{A}_{i}(0)\right]\left(\frac{1}{\alpha} \psi_{A}\right)(x)\right]\left[\bar{\psi}_{B}(0) \not p_{1}\left[B^{i}(x)-i \gamma_{5} \tilde{B}^{i}(x)\right]\left(\frac{1}{\beta} \psi_{B}\right)(0)\right]\right\} \\
& -2 a\left\{\left[\left(\bar{\psi}_{A} \frac{1}{\alpha}\right)(x) \not p_{2}\left[A_{i}(0)+i \gamma_{5} \tilde{A}_{i}(0)\right] \psi_{A}(x)\right]\left[\left(\bar{\psi}_{B} \frac{1}{\beta}\right)(0) \not p_{1}\left[\gamma_{5} B^{i}(x)+i \tilde{B}^{i}(x)\right] \psi_{B}(0)\right]\right. \\
& \left.\left.+\left[\bar{\psi}_{A}(x) \not p_{2}\left[A_{i}(0)-i \gamma_{5} \tilde{A}_{i}(0)\right]\left(\frac{1}{\alpha} \psi_{A}\right)(x)\right]\left[\bar{\psi}_{B}(0) \not p_{1}\left[\gamma_{5} B^{i}(x)-i \tilde{B}^{i}(x)\right]\left(\frac{1}{\beta} \psi_{B}\right)(0)\right]\right\}\right) \\
& +x \leftrightarrow 0 .
\end{aligned}
$$

Using eq. (4.58) one obtains the contribution to $W\left(\alpha_{q}, \beta_{q}, x_{\perp}\right)$ in the form

$$
\begin{aligned}
& -\frac{g^{2} e^{2}\left(a^{2}+1\right)}{8(2 \pi)^{4} s_{W}^{2} c_{W}^{2}\left(N_{c}^{2}-1\right) Q^{2} s^{2}} \int d x_{\bullet} d x_{*} e^{-i \alpha_{q} x_{\bullet}-i \beta_{q} x_{*}} \\
& \times\left\{\int _ { - \infty } ^ { x } d x _ { \bullet } ^ { \prime } \int _ { - \infty } ^ { 0 } d x _ { * } ^ { \prime } \left[\left\langle A\left|\hat{\bar{\psi}}\left(x_{\bullet}^{\prime}, x_{\perp}\right) \frac{2 \not p_{2}}{s}\left[\hat{F}_{* i}(0)+i \gamma_{5} \hat{\tilde{F}}_{* i}(0)\right] \hat{\psi}\left(x_{\bullet}, x_{\perp}\right)\right| A\right\rangle\right.\right. \\
& \quad \times\left\langle B\left|\hat{\bar{\psi}}\left(x_{*}^{\prime}, 0_{\perp}\right) \frac{2 \not p_{1}}{s}\left[\hat{F}_{\bullet}^{i}\left(x_{*}, x_{\perp}\right)+i \gamma_{5} \hat{\tilde{F}}_{\bullet}^{i}\left(x_{*}, x_{\perp}\right)\right] \hat{\psi}(0)\right| B\right\rangle \\
& \quad+\left\langle A\left|\hat{\bar{\psi}}\left(x_{\bullet}, x_{\perp}\right) \frac{2 \not p_{2}}{s}\left[\hat{F}_{* i}(0)-i \gamma_{5} \hat{\tilde{F}}_{* i}(0)\right] \hat{\psi}\left(x_{\bullet}^{\prime}, x_{\perp}\right)\right| A\right\rangle \\
& \left.\left.\quad \times\left\langle B\left|\hat{\bar{\psi}}(0) \frac{2 \not p_{1}}{s}\left[\hat{F}_{\bullet}^{i}\left(x_{*}, x_{\perp}\right)-i \gamma_{5} \hat{\tilde{F}}_{\bullet}^{i}\left(x_{*}, x_{\perp}\right)\right] \hat{\psi}\left(x_{*}^{\prime}, 0_{\perp}\right)\right| B\right\rangle\right]+x \leftrightarrow 0\right\} .
\end{aligned}
$$


Here we used the fact that the last term in eq. $(4.60)\left(\sim \frac{2 a}{s^{2}}\right)$

$$
\begin{aligned}
-2 a & {\left[A\left|\hat{\bar{\psi}}\left(x_{\bullet}^{\prime}, x_{\perp}\right) \frac{2 \not p_{2}}{s}\left[\hat{F}_{* i}(0)+i \gamma_{5} \hat{\tilde{F}}_{* i}(0)\right] \hat{\psi}\left(x_{\bullet}, x_{\perp}\right)\right| A\right\rangle } \\
& \times\left\langle B\left|\hat{\bar{\psi}}\left(x_{*}^{\prime}, 0_{\perp}\right) \frac{2 \not p_{1}}{s}\left[\gamma_{5} \hat{F}_{\bullet}^{i}\left(x_{*}, x_{\perp}\right)+i \hat{\tilde{F}}_{\bullet}^{i}\left(x_{*}, x_{\perp}\right)\right] \hat{\psi}(0)\right| B\right\rangle \\
& +\left\langle A\left|\hat{\bar{\psi}}\left(x_{\bullet}, x_{\perp}\right) \frac{2 \not p_{2}}{s}\left[\hat{F}_{* i}(0)-i \gamma_{5} \hat{\tilde{F}}_{* i}(0)\right] \hat{\psi}\left(x_{\bullet}^{\prime}, x_{\perp}\right)\right| A\right\rangle \\
& \left.\times\left\langle B\left|\hat{\bar{\psi}}(0) \frac{2 \not p_{1}}{s}\left[\gamma_{5} \hat{F}_{\bullet}^{i}\left(x_{*}, x_{\perp}\right)-i \hat{\tilde{F}}_{\bullet}^{i}\left(x_{*}, x_{\perp}\right)\right] \hat{\psi}\left(x_{*}^{\prime}, 0_{\perp}\right)\right| B\right\rangle\right]
\end{aligned}
$$

gives no contribution since

$$
\begin{aligned}
\left\langle A\left|\hat{\bar{\psi}}\left(x_{\bullet}^{\prime}, x_{\perp}\right) \frac{2 \not p_{2}}{s}\left[\hat{F}_{* i}(0) \pm i \gamma_{5} \hat{\tilde{F}}_{* i}(0)\right] \hat{\psi}\left(x_{\bullet}, x_{\perp}\right)\right| A\right\rangle & \sim x_{i}, \\
\left\langle B\left|\hat{\bar{\psi}}\left(x_{*}^{\prime}, 0_{\perp}\right) \frac{2 \not p_{1}}{s}\left[\gamma_{5} \hat{F}_{\bullet i}\left(x_{*}, x_{\perp}\right) \pm i \hat{\tilde{F}}_{\bullet i}\left(x_{*}, x_{\perp}\right)\right] \hat{\psi}(0)\right| B\right\rangle & \sim \epsilon_{i j} x^{j}
\end{aligned}
$$

same as in eq. (4.27).

Next, using parametrizations (4.66) from the next section we obtain the contribution of the 6 th and 7 th lines in eq. (4.56) in the form

$$
\begin{aligned}
W^{6+} & 7 \operatorname{th}\left(\alpha_{q}, \beta_{q}, q_{\perp}\right)=\frac{e^{2}}{8 s_{W}^{2} c_{W}^{2}\left(N_{c}^{2}-1\right) Q^{2}} \int d^{2} k_{\perp}(k, q-k)_{\perp}\left[\left\{2\left(1+a_{u}^{2}\right)\right.\right. \\
& \times\left[j_{1 u}^{\mathrm{tw} 3}\left(\alpha_{q}, k_{\perp}\right) j_{2 u}^{\mathrm{tw} 3}\left(\beta_{q}, q_{\perp}-k_{\perp}\right)-\tilde{j}_{1 u}^{\mathrm{tw} 3}\left(\alpha_{q}, k_{\perp}\right) \tilde{j}_{2 u}^{\mathrm{tw} 3}\left(\beta_{q}, q_{\perp}-k_{\perp}\right)\right] \\
& +\left(1-a_{u}^{2}\right)\left[j_{1 u}^{\mathrm{tw} 3}\left(\alpha_{q}, k_{\perp}\right) j_{1 u}^{\mathrm{tw} 3}\left(\beta_{q}, q_{\perp}-k_{\perp}\right)+\tilde{j}_{1 u}^{\mathrm{tw} 3}\left(\alpha_{q}, k_{\perp}\right) \tilde{j}_{1 u}^{\mathrm{tw} 3}\left(\beta_{q}, q_{\perp}-k_{\perp}\right)\right. \\
& \left.\left.+j_{2 u}^{\mathrm{tw} 3}\left(\alpha_{q}, k_{\perp}\right) j_{2 u}^{\mathrm{tw} 3}\left(\beta_{q}, q_{\perp}-k_{\perp}\right)+\tilde{j}_{2 u}^{\mathrm{tw} 3}\left(\alpha_{q}, k_{\perp}\right) \tilde{j}_{2 u}^{\mathrm{tw} 3}\left(\beta_{q}, q_{\perp}-k_{\perp}\right)\right]+\alpha_{q} \leftrightarrow \beta_{q}\right\} \\
& +\{u \leftrightarrow c\}+\{u \leftrightarrow d\}+\{u \leftrightarrow s\}]\left(1+O\left(\frac{m_{\perp}^{2}}{s}\right)\right),
\end{aligned}
$$

where $\alpha_{q} \leftrightarrow \beta_{q}$ contribution comes as usually from the $(x \leftrightarrow 0)$ term in eq. (4.59).

\subsubsection{Parametrization of TMDs from section 4.4.1}

We parametrize TMDs from section 4.4.1 as follows

$$
\begin{aligned}
& \frac{g}{8 \pi^{3} s} \int d^{2} x_{\perp} d x \bullet e^{-i \alpha x \bullet+i(k, x)_{\perp}} \int_{-\infty}^{x \bullet} d x_{\bullet}^{\prime}\left\langle A\left|\hat{\bar{\psi}}\left(x_{\bullet}^{\prime}, x_{\perp}\right) \frac{2 \not p_{2}}{s}\left[\hat{F}_{* i}(0)+i \gamma_{5} \hat{\tilde{F}}_{* i}(0)\right] \hat{\psi}\left(x_{\bullet}, x_{\perp}\right)\right| A\right\rangle \\
& \quad=k_{i}\left[j_{1}^{\mathrm{tw} 3}\left(\alpha, k_{\perp}^{2}\right)+i \tilde{j}_{1}^{\mathrm{tw} 3}\left(\alpha, k_{\perp}^{2}\right)\right], \\
& \frac{g}{8 \pi^{3} s} \int d^{2} x_{\perp} d x \bullet e^{-i \alpha x \bullet}+i(k, x)_{\perp} \int_{-\infty}^{x} d_{\bullet}^{\prime}\left\langle A\left|\hat{\bar{\psi}}\left(x_{\bullet}, x_{\perp}\right) \frac{2 \not p_{2}}{s}\left[\hat{F}_{* i}(0)-i \gamma_{5} \hat{\tilde{F}}_{* i}(0)\right] \hat{\psi}\left(x_{\bullet}^{\prime}, x_{\perp}\right)\right| A\right\rangle \\
& \quad=k_{i}\left[j_{2}^{\mathrm{tw} 3}\left(\alpha, k_{\perp}^{2}\right)-i \tilde{j}_{2}^{\mathrm{tw} 3}\left(\alpha, k_{\perp}^{2}\right)\right] .
\end{aligned}
$$


By complex conjugation we get

$$
\begin{aligned}
& \frac{g}{8 \pi^{3} s} \int d^{2} x_{\perp} d x_{\bullet} e^{-i \alpha x_{\bullet}+i(k, x)_{\perp}} \int_{-\infty}^{0} d x_{\bullet}^{\prime}\left\langle A\left|\overline{\hat{\psi}}(0) \frac{2 \not p_{2}}{s}\left[\hat{F}_{* i}(x)-i \gamma_{5} \hat{\tilde{F}}_{* i}(x)\right] \hat{\psi}\left(x_{\bullet}^{\prime}, 0_{\perp}\right)\right| A\right\rangle \\
& \quad=k_{i}\left[j_{1}^{\mathrm{tw} 3}\left(\alpha, k_{\perp}^{2}\right)-i \tilde{j}_{1}^{\mathrm{tw} 3}\left(\alpha, k_{\perp}^{2}\right)\right], \\
& \frac{g}{8 \pi^{3} s} \int d^{2} x_{\perp} d x_{\bullet} e^{-i \alpha x_{\bullet}+i(k, x)_{\perp}} \int_{-\infty}^{0} d x_{\bullet}^{\prime}\left\langle A\left|\overline{\hat{\psi}}\left(x_{\bullet}^{\prime}, 0_{\perp}\right) \frac{2 \not p_{2}}{s}\left[\hat{F}_{* i}(x)+i \gamma_{5} \hat{\tilde{F}}_{* i}(x)\right] \hat{\psi}(0)\right| A\right\rangle \\
& \quad=k_{i}\left[j_{2}^{\mathrm{tw} 3}\left(\alpha, k_{\perp}^{2}\right)+i \tilde{j}_{2}^{\mathrm{tw} 3}\left(\alpha, k_{\perp}^{2}\right)\right] .
\end{aligned}
$$

Target matrix elements are obtained by usual substitutions $\alpha \leftrightarrow \beta, \not p_{2} \leftrightarrow \not p_{1}, x_{\bullet} \leftrightarrow x_{*}$, and $\hat{F}_{* i} \leftrightarrow \hat{F}_{\bullet i}$.

For completeness let us present the explicit form of the gauge links in an arbitrary gauge:

$$
\begin{aligned}
& \hat{\bar{\psi}}\left(x_{\bullet}^{\prime}, x_{\perp}\right) \hat{F}_{* i}(0) \hat{\psi}\left(x_{\bullet}, x_{\perp}\right) \rightarrow \\
& \hat{\bar{\psi}}\left(x_{\bullet}^{\prime}, x_{\perp}\right)\left[x_{\bullet}^{\prime},-\infty_{\bullet}\right]_{x}\left[x_{\perp}, 0_{\perp}\right]_{-\infty}\left[-\infty_{\bullet}, 0\right]_{0_{\perp}} \hat{F}_{* i}(0)\left[0,-\infty_{\bullet}\right]_{0_{\perp}}\left[0_{\perp}, x_{\perp}\right]_{-\infty}\left[-\infty_{\bullet}, x_{\bullet}\right]_{x} \hat{\psi}\left(x_{\bullet}, x_{\perp}\right) .
\end{aligned}
$$

\section{$5 \quad$ Results and estimates}

Combining eqs. (4.12), (4.31), (4.51), and (4.64) we get the leading term and first power corrections to $W(q)$ in the kinematic region $s \gg Q^{2} \gg q_{\perp}^{2}$ in the form

$$
\begin{aligned}
& W\left(p_{A}, p_{B}, q\right)=-\frac{e^{2}}{8 s_{W}^{2} c_{W}^{2} N_{c}} \int d^{2} k_{\perp}\left[\left\{\left(1+a_{u}^{2}\right)\left[1-2 \frac{(k, q-k)_{\perp}}{Q^{2}}\right]\right.\right. \\
& \times f_{1 u}\left(\alpha_{z}, k_{\perp}\right) \bar{f}_{1 u}\left(\beta_{z}, q_{\perp}-k_{\perp}\right)+2\left(a_{u}^{2}-1\right) \frac{k_{\perp}^{2}(q-k)_{\perp}^{2}}{m_{N}^{2} Q^{2}} h_{1 u}^{\perp}\left(\alpha_{z}, k_{\perp}\right) \bar{h}_{1 u}^{\perp}\left(\beta_{z}, q_{\perp}-k_{\perp}\right) \\
& +\frac{2 k_{\perp}^{2}(q-k)_{\perp}^{2}}{\left(N_{c}^{2}-1\right) Q^{2} m_{N}^{2}}\left(a_{u}^{2}-1\right)\left[h_{u}^{\mathrm{tw} 3}\left(\alpha_{z}, k_{\perp}\right) \bar{h}_{u}^{\mathrm{tw} 3}\left(\beta_{z}, q_{\perp}-k_{\perp}\right)+\tilde{h}_{u}^{\mathrm{tw} 3}\left(\alpha_{z}, k_{\perp}\right) \tilde{\bar{h}}_{u}^{\mathrm{tw} 3}\left(\beta_{z}, q_{\perp}-k_{\perp}\right)\right] \\
& -\frac{N_{c}}{N_{c}^{2}-1} \frac{(k, q-k)_{\perp}}{Q^{2}}\left(2\left(1+a_{u}^{2}\right)\left[j_{1 u}^{\mathrm{tw} 3}\left(\alpha_{z}, k_{\perp}\right) j_{2 u}^{\mathrm{tw} 3}\left(\beta_{z}, q_{\perp}-k_{\perp}\right)-\tilde{j}_{1 u}^{\mathrm{tw} 3}\left(\alpha_{z}, k_{\perp}\right) \tilde{j}_{2 u}^{\mathrm{tw} 3}\left(\beta_{z}, q_{\perp}-k_{\perp}\right)\right]\right. \\
& \quad+\left(1-a_{u}^{2}\right)\left[j_{1 u}^{\mathrm{tw} 3}\left(\alpha_{z}, k_{\perp}\right) j_{1 u}^{\mathrm{tw} 3}\left(\beta_{z}, q_{\perp}-k_{\perp}\right)+j_{2 u}^{\mathrm{tw} 3}\left(\alpha_{z}, k_{\perp}\right) j_{2 u}^{\mathrm{tw} 3}\left(\beta_{z}, q_{\perp}-k_{\perp}\right)\right. \\
& \left.\left.\quad+\tilde{j}_{1 u}^{\mathrm{tw} 3}\left(\alpha_{z}, k_{\perp}\right) \tilde{j}_{1 u}^{\mathrm{tw} 3}\left(\beta_{z}, q_{\perp}-k_{\perp}\right)+\tilde{j}_{2 u}^{\mathrm{tw} 3}\left(\alpha_{z}, k_{\perp}\right) \tilde{j}_{2 u}^{\mathrm{tw} 3}\left(\beta_{z}, q_{\perp}-k_{\perp}\right)\right]\right) \\
& \left.\left.+\left(\alpha_{z} \leftrightarrow \beta_{z}\right)\right\}+\{u \leftrightarrow c\}+\{u \leftrightarrow d\}+\{u \leftrightarrow s\}\right]\left(1+O\left(\frac{m_{\perp}^{2}}{s}\right)\right),
\end{aligned}
$$

where the momentum of the produced Z-boson is $q=\alpha_{z} p_{1}+\beta_{z} p_{2}+q_{\perp}$. 
For completeness, let us present our final result in the transverse coordinate space

$$
\begin{gathered}
W\left(p_{A}, p_{B}, q\right)=-\frac{e^{2}}{8 s_{W}^{2} c_{W}^{2} N_{c}} \int \frac{d^{2} b_{\perp}}{4 \pi^{2}} e^{i(q, b)_{\perp}}\left[\left\{( 1 + a _ { u } ^ { 2 } ) \left[f_{1 u}\left(\alpha_{z}, b_{\perp}\right) \bar{f}_{1 u}\left(\beta_{z}, b_{\perp}\right)\right.\right.\right. \\
\left.+\frac{2}{Q^{2}} \partial_{i}^{\perp} f_{1 u}\left(\alpha_{z}, b_{\perp}\right) \partial_{i}^{\perp} \bar{f}_{1 u}\left(\beta_{z}, b_{\perp}\right)\right]+\frac{2\left(a_{u}^{2}-1\right)}{m_{N}^{2} Q^{2}} \partial_{\perp}^{2} h_{1 u}^{\perp}\left(\alpha_{z}, b_{\perp}\right) \partial_{\perp}^{2} \bar{h}_{1 u}^{\perp}\left(\beta_{z}, b_{\perp}\right) \\
+\frac{2\left(a_{u}^{2}-1\right)}{\left(N_{c}^{2}-1\right) Q^{2} m_{N}^{2}}\left[\partial_{\perp}^{2} h_{u}^{\mathrm{tw} 3}\left(\alpha_{z}, b_{\perp}\right) \partial_{\perp}^{2} \bar{h}_{u}^{\mathrm{tw} 3}\left(\beta_{z}, b_{\perp}\right)+\partial_{\perp}^{2} \tilde{h}_{u}^{\mathrm{tw} 3}\left(\alpha_{z}, b_{\perp}\right) \partial_{\perp}^{2} \tilde{\bar{h}}_{u}^{\mathrm{tw} 3}\left(\beta_{z}, b_{\perp}\right)\right] \\
+\frac{N_{c}}{\left(N_{c}^{2}-1\right) Q^{2}}\left(2\left(1+a_{u}^{2}\right)\left[\partial_{i}^{\perp} j_{1 u}^{\mathrm{tw} 3}\left(\alpha_{z}, b_{\perp}\right) \partial_{i}^{\perp} j_{2 u}^{\mathrm{tw} 3}\left(\beta_{z}, b_{\perp}\right)-\partial_{i}^{\perp} \tilde{j}_{1 u}^{\mathrm{tw} 3}\left(\alpha_{z}, b_{\perp}\right) \partial_{i}^{\perp} \tilde{j}_{2 u}^{\mathrm{tw} 3}\left(\beta_{z}, b_{\perp}\right)\right]\right. \\
+\left(1-a_{u}^{2}\right)\left[\partial_{i}^{\perp} j_{1 u}^{\mathrm{tw} 3}\left(\alpha_{z}, b_{\perp}\right) \partial_{i}^{\perp} j_{1 u}^{\mathrm{tw} 3}\left(\beta_{z}, b_{\perp}\right)+\partial_{i}^{\perp} j_{2 u}^{\mathrm{tw} 3}\left(\alpha_{z}, b_{\perp}\right) \partial_{i}^{\perp} j_{2 u}^{\mathrm{tw} 3}\left(\beta_{z}, b_{\perp}\right)\right. \\
\left.\left.+\partial_{i}^{\perp} \tilde{j}_{1 u}^{\mathrm{tw} 3}\left(\alpha_{z}, b_{\perp}\right) \partial_{i}^{\perp} \tilde{j}_{1 u}^{\mathrm{tw} 3}\left(\beta_{z}, b_{\perp}\right)+\partial_{i}^{\perp} \tilde{j}_{2 u}^{\mathrm{tw} 3}\left(\alpha_{z}, b_{\perp}\right) \partial_{i}^{\perp} \tilde{j}_{2 u}^{\mathrm{tw} 3}\left(\beta_{z}, b_{\perp}\right)\right]\right) \\
\left.\left.+\left(\alpha_{z} \leftrightarrow \beta_{z}\right)\right\}+\{u \leftrightarrow c\}+\{u \leftrightarrow d\}+\{u \leftrightarrow s\}\right]\left(1+O\left(\frac{m_{\perp}^{2}}{s}\right)\right),
\end{gathered}
$$

where $f_{1 u}\left(\alpha_{z}, b_{\perp}\right) \equiv \int d^{2} k_{\perp} e^{-i(k, b)} f_{1 u}\left(\alpha_{z}, k_{\perp}\right)$ etc.

Note that in the leading order in $N_{c}$ power corrections are expressed in terms of leading power functions $f_{1}$ and $h_{1}^{\perp}$. To estimate the order of magnitude of power corrections, one can assume that $\frac{1}{N_{c}}$ is a good parameter and leave only first term in the r.h.s. of eq. (5.1):

$$
\begin{aligned}
& W\left(p_{A}, p_{B}, q\right)=-\frac{e^{2}}{8 s_{W}^{2} c_{W}^{2} N_{c}} \int d^{2} k_{\perp}\left[\left\{\left(1+a_{u}^{2}\right)\left[1-2 \frac{(k, q-k)_{\perp}}{Q^{2}}\right]\right.\right. \\
& \times f_{1 u}\left(\alpha_{z}, k_{\perp}\right) \bar{f}_{1 u}\left(\beta_{z}, q_{\perp}-k_{\perp}\right)+2\left(a_{u}^{2}-1\right) \frac{k_{\perp}^{2}(q-k)_{\perp}^{2}}{m_{N}^{2} Q^{2}} h_{1 u}^{\perp}\left(\alpha_{z}, k_{\perp}\right) \bar{h}_{1 u}^{\perp}\left(\beta_{z}, q_{\perp}-k_{\perp}\right) \\
& \left.\left.\quad+\left(\alpha_{z} \leftrightarrow \beta_{z}\right)\right\}+\{u \leftrightarrow c\}+\{u \leftrightarrow d\}+\{u \leftrightarrow s\}\right]\left(1+O\left(\frac{m_{\perp}^{2}}{s}\right)+O\left(\frac{1}{N_{c}}\right)\right) .
\end{aligned}
$$

Next, eq. (5.3) is a tree-level formula and for an estimate we should specify the rapidity cutoffs for $f_{1}$ 's and $h_{1}^{\perp}$ 's. As we discussed in section 2 , the rapidity cutoff for $f_{1}\left(\alpha_{z}, k_{\perp}^{2}\right)$ is $\sigma_{a}$ and for $f_{1}\left(\alpha_{z}, k_{\perp}^{2}\right) \sigma_{b}$, where $\sigma_{a}$ and $\sigma_{b}$ are rapidity bounds for central fields. Since we calculated only tree diagrams made of $C$-fields we have $\sigma_{a}=\beta_{z}$ and $\sigma_{b}=\alpha_{z}$ in eq. (5.1). ${ }^{15}$

Next, power corrections become sizable at $q_{\perp}^{2} \gg m_{N}^{2}$ where we probe the perturbative tails of TMD's $f_{1} \sim \frac{1}{k_{\perp}^{2}}$ and $h_{1}^{\perp} \sim \frac{1}{k_{\perp}^{4}}$ [34]. So, as long as $m_{N}^{2} \ll k_{\perp}^{2} \ll Q^{2}$ we can approximate

$$
f_{1}\left(\alpha_{z}, k_{\perp}^{2}\right) \simeq \frac{f\left(\alpha_{z}\right)}{k_{\perp}^{2}}, \quad h_{1}^{\perp}\left(\alpha_{z}, k_{\perp}^{2}\right) \simeq \frac{m_{N}^{2} h\left(\alpha_{z}\right)}{k_{\perp}^{4}}, \quad \bar{f}_{1} \simeq \frac{\bar{f}\left(\alpha_{z}\right)}{k_{\perp}^{2}}, \quad \bar{h}_{1}^{\perp} \simeq \frac{m_{N}^{2} \bar{h}\left(\alpha_{z}\right)}{k_{\perp}^{4}}
$$

(up to logarithmic corrections). Similarly, for the target we can use the estimate

$$
f_{1}\left(\beta_{z}, k_{\perp}^{2}\right) \simeq \frac{f\left(\beta_{z}\right)}{k_{\perp}^{2}}, \quad h_{1}^{\perp}\left(\beta_{z}, k_{\perp}^{2}\right) \simeq \frac{m_{N}^{2} h\left(\beta_{z}\right)}{k_{\perp}^{4}}, \quad \bar{f}_{1} \simeq \frac{\bar{f}\left(\beta_{z}\right)}{k_{\perp}^{2}}, \quad \bar{h}_{1}^{\perp} \simeq \frac{m_{N}^{2} \bar{h}\left(\beta_{z}\right)}{k_{\perp}^{4}}
$$

as long as $m_{N}^{2} \ll k_{\perp}^{2} \ll Q^{2}$.

\footnotetext{
${ }^{15}$ In general, we should integrate over $C$-fields in the leading log approximation and match the logs to the double-log and/or single-log evolution of TMDs.
} 
Substituting this to eq. (5.1) we get the following estimate of the strength of power corrections for Z-boson production

$$
\begin{aligned}
& W\left(p_{A}, p_{B}, q\right)=-\frac{e^{2}}{8 s_{W}^{2} c_{W}^{2} N_{c}} \int d^{2} k_{\perp} \frac{1}{k_{\perp}^{2}(q-k)_{\perp}^{2}} \\
& \quad \times\left[\left\{\left(1+a_{u}^{2}\right)\left[1-2 \frac{(k, q-k)_{\perp}}{Q^{2}}\right]\left[f_{u}\left(\alpha_{z}\right) \bar{f}_{u}\left(\beta_{z}\right)+\bar{f}_{u}\left(\alpha_{z}\right) f_{u}\left(\beta_{z}\right)\right]\right.\right. \\
& \left.\left.\quad+2\left(a_{u}^{2}-1\right)\left[h_{u}\left(\alpha_{z}\right) \bar{h}_{u}\left(\beta_{z}\right)+\bar{h}_{u}\left(\alpha_{z}\right) h_{u}\left(\beta_{z}\right)\right] \frac{m_{N}^{2}}{Q^{2}}\right\}+\{u \leftrightarrow c\}+\{u \leftrightarrow d\}+\{u \leftrightarrow s\}\right] \\
& \simeq-\frac{e^{2}}{8 s_{W}^{2} c_{W}^{2} N_{c}} \int d^{2} k_{\perp} \frac{1}{k_{\perp}^{2}(q-k)_{\perp}^{2}}\left[1-2 \frac{(k, q-k)_{\perp}}{Q^{2}}\right] \\
& \quad \times\left[\left\{\left(1+a_{u}^{2}\right)\left[f_{u}\left(\alpha_{z}\right) \bar{f}_{u}\left(\beta_{z}\right)+\bar{f}_{u}\left(\alpha_{z}\right) f_{u}\left(\beta_{z}\right)\right]\right\}+\{u \leftrightarrow c\}+\{u \leftrightarrow d\}+\{u \leftrightarrow s\}\right] .
\end{aligned}
$$

Here we used the fact that due to the "positivity constraint" $h_{1}^{\perp}\left(x, k_{\perp}^{2}\right) \leq \frac{m_{N}}{\left|k_{\perp}\right|} f_{1}^{\perp}\left(x, k_{\perp}^{2}\right)[35]$, we can safely assume that the numbers $f(x)$ and $h(x)$ in eqs. (5.4) and (5.5) are of the same order of magnitude so the last term in the third line in eq. (5.6) $\sim \frac{m_{N}^{2}}{Q^{2}}$ can be neglected. Thus, the relative weight of the leading term and power correction is determined by the factor $1-2 \frac{(k, q-k)_{\perp}}{Q^{2}}$. The integrals over $k_{\perp}$ are logarithmic and should be cut from below by $m_{N}^{2}$ and from above by $Q^{2}$ so we get an estimate

$$
\begin{aligned}
& W\left(p_{A}, p_{B}, q\right)=-\frac{\pi e^{2}}{4 s_{W}^{2} c_{W}^{2} N_{c}}\left[\frac{1}{q_{\perp}^{2}} \ln \frac{q_{\perp}^{2}}{m_{N}^{2}}+\frac{1}{Q^{2}} \ln \frac{Q^{2}}{q_{\perp}^{2}}\right] \\
& \times\left[\left\{\left(1+a_{u}^{2}\right)\left[f_{u}\left(\alpha_{z}\right) \bar{f}_{u}\left(\beta_{z}\right)+\bar{f}_{u}\left(\alpha_{z}\right) f_{u}\left(\beta_{z}\right)\right]\right\}+\{u \leftrightarrow c\}+\{u \leftrightarrow d\}+\{u \leftrightarrow s\}\right],
\end{aligned}
$$

where we assumed that the first term is determined by the logarithmical region $q_{\perp}^{2} \gg k_{\perp}^{2} \gg m_{N}^{2}$ and the second by $Q^{2} \gg k_{\perp}^{2} \gg q_{\perp}^{2}$. By this estimate, the power correction reaches the level of few percent at $q_{\perp} \geq 20 \mathrm{GeV}$. Of course, when $q_{\perp}^{2}$ increases, the correction becomes bigger, but the validity of the approximation $\frac{q_{\perp}^{2}}{Q^{2}} \ll 1$ worsens. Moreover, we have ignored all logarithmic (and double-log) evolutions which can significantly change the relative strength of power corrections.

\section{Power corrections for Drell-Yan process}

In this section we consider $\gamma^{*}$ contribution to the cross section of the Drell-Yan process which is determined by the hadronic tensor

$$
\begin{aligned}
W_{\mu \nu}\left(p_{A}, p_{B}, q\right) & =\int d^{2} x_{\perp} e^{i(q, x)_{\perp}} W_{\mu \nu}\left(\alpha_{q}, \beta_{q}, x_{\perp}\right) \\
W_{\mu \nu}\left(\alpha_{q}, \beta_{q}, x_{\perp}\right) & \equiv \frac{1}{16 \pi^{4}} \frac{2}{s} \int d x_{\bullet} d x_{*} e^{-i \alpha_{q} x_{\bullet}-i \beta_{q} x_{*}}\left\langle p_{A}, p_{B}\left|J_{\mu}^{\mathrm{em}}\left(x_{\bullet}, x_{*}, x_{\perp}\right) J_{\nu}^{\mathrm{em}}(0)\right| p_{A}, p_{B}\right\rangle
\end{aligned}
$$

where $J_{\mu}^{\mathrm{em}}=e_{u} \bar{\psi}_{u} \gamma_{\mu} \psi_{u}+e_{d} \bar{\psi}_{d} \gamma_{\mu} \psi_{d}+e_{s} \bar{\psi}_{s} \gamma_{\mu} \psi_{s}+e_{c} \bar{\psi}_{c} \gamma_{\mu} \psi_{c}$ is the electromagnetic current for active flavors in our kinematical region. 
From the results of the present paper it is easy to extract power corrections to $W_{\mu}^{\mu} \cdot{ }^{16}$ We replace constants $a_{u}$ in eq. (5.1) by $e_{f}^{2}$ and remove factors "1" from expressions like $a^{2} \pm 1$. One can formally set $a_{u} \rightarrow \infty$ in $\breve{\gamma}_{\mu} \equiv \gamma_{\mu}\left(a_{u}-\gamma_{5}\right)$, divide the result (5.1) by $a_{u}^{2}$, and multiply by $e_{u}^{2}$. After that, we repeat the procedure for other flavors and get

$$
\begin{aligned}
& W_{\mu}^{\mu}\left(p_{A}, p_{B}, q\right)=-\frac{2}{N_{c}} \int d^{2} k_{\perp}\left[\left\{e_{u}^{2}\left[1-2 \frac{(k, q-k)_{\perp}}{Q^{2}}\right] f_{1 u}\left(\alpha_{q}, k_{\perp}\right) \bar{f}_{1 u}\left(\beta_{q}, q_{\perp}-k_{\perp}\right)\right.\right. \\
& +2 e_{u}^{2} \frac{k_{\perp}^{2}(q-k)_{\perp}^{2}}{m_{N}^{2} Q^{2}}\left[h_{1 u}^{\perp}\left(\alpha_{q}, k_{\perp}\right) \bar{h}_{1 u}^{\perp}\left(\beta_{q}, q_{\perp}-k_{\perp}\right)\right. \\
& \left.+\frac{1}{N_{c}^{2}-1}\left(h_{u}^{\mathrm{tw} 3}\left(\alpha_{q}, k_{\perp}\right) \bar{h}_{u}^{\mathrm{tw} 3}\left(\beta_{q}, q_{\perp}-k_{\perp}\right)+\tilde{h}_{u}^{\mathrm{tw} 3}\left(\alpha_{q}, k_{\perp}\right) \tilde{\bar{h}}_{u}^{\mathrm{tw} 3}\left(\beta_{q}, q_{\perp}-k_{\perp}\right)\right)\right] \\
& -e_{u}^{2} \frac{N_{c}}{N_{c}^{2}-1} \frac{(k, q-k)_{\perp}}{Q^{2}}\left[2 j_{1 u}^{\mathrm{tw} 3}\left(\alpha_{q}, k_{\perp}\right) j_{2 u}^{\mathrm{tw} 3}\left(\beta_{q}, q_{\perp}-k_{\perp}\right)-2 \tilde{j}_{1 u}^{\mathrm{tw} 3}\left(\alpha_{q}, k_{\perp}\right) \tilde{j}_{2 u}^{\mathrm{tw} 3}\left(\beta_{q}, q_{\perp}-k_{\perp}\right)\right. \\
& -j_{1 u}^{\mathrm{tw} 3}\left(\alpha_{q}, k_{\perp}\right) j_{1 u}^{\mathrm{tw} 3}\left(\beta_{q}, q_{\perp}-k_{\perp}\right)-j_{2 u}^{\mathrm{tw} 3}\left(\alpha_{q}, k_{\perp}\right) j_{2 u}^{\mathrm{tw} 3}\left(\beta_{q}, q_{\perp}-k_{\perp}\right) \\
& \left.\left.-\tilde{j}_{1 u}^{\mathrm{tw} 3}\left(\alpha_{q}, k_{\perp}\right) \tilde{j}_{1 u}^{\mathrm{tw} 3}\left(\beta_{q}, q_{\perp}-k_{\perp}\right)-\tilde{j}_{2 u}^{\mathrm{tw} 3}\left(\alpha_{q}, k_{\perp}\right) \tilde{j}_{2 u}^{\mathrm{tw} 3}\left(\beta_{q}, q_{\perp}-k_{\perp}\right)\right]+\left(\alpha_{q} \leftrightarrow \beta_{q}\right)\right\} \\
& +\{u \leftrightarrow c\}+\{u \leftrightarrow d\}+\{u \leftrightarrow s\}] .
\end{aligned}
$$

Let us present also the large- $N_{c}$ estimate similar to eq. (5.6)

$$
\begin{aligned}
W_{\mu}^{\mu}\left(p_{A}, p_{B}, q\right)= & -\frac{2}{N_{c}} \int d^{2} k_{\perp} \frac{1}{k_{\perp}^{2}(q-k)_{\perp}^{2}} \\
& \times\left[\left\{e_{u}^{2}\left[1-2 \frac{(k, q-k)_{\perp}}{Q^{2}}\right]\left[f_{u}\left(\alpha_{q}\right) \bar{f}_{u}\left(\beta_{q}\right)+\bar{f}_{u}\left(\alpha_{q}\right) f_{u}\left(\beta_{q}\right)\right]\right.\right. \\
& \left.\left.+2 e_{u}^{2}\left[h_{u}\left(\alpha_{q}\right) \bar{h}_{u}\left(\beta_{q}\right)+\bar{h}_{u}\left(\alpha_{q}\right) h_{u}\left(\beta_{q}\right)\right] \frac{m_{N}^{2}}{Q^{2}}\right\}+\{u \leftrightarrow c\}+\{u \leftrightarrow d\}+\{u \leftrightarrow s\}\right] \\
\simeq & -\frac{2}{N_{c}} \int d^{2} k_{\perp} \frac{1}{k_{\perp}^{2}(q-k)_{\perp}^{2}}\left[1-2 \frac{(k, q-k)_{\perp}}{Q^{2}}\right] \\
& \times\left\{e_{u}^{2}\left[f_{u}\left(\alpha_{q}\right) \bar{f}_{u}\left(\beta_{q}\right)+\bar{f}_{u}\left(\alpha_{q}\right) f_{u}\left(\beta_{q}\right)\right]+(u \leftrightarrow c)+(u \leftrightarrow d)+(u \leftrightarrow s)\right\} .
\end{aligned}
$$

Obviously, the relative strength of leading-twist terms and power corrections is the same as for Z-boson production so from our naïve estimate (5.7) one should expect power corrections of order of few percent starting from $q_{\perp} \sim \frac{1}{4} Q$.

\section{Conclusions and outlook}

In this paper we have calculated the higher-twist power correction to Z-boson production (and Drell-Yan process) in the kinematical region $s \gg Q^{2} \gg q_{\perp}^{2}$. Our back-of-the-envelope estimation of importance of power corrections tells that they reach a few percent of the leading-twist result at $q_{\perp} \sim \frac{1}{4} Q$ which surprisingly agrees with the same estimate made in ref. [21] by comparing leading-order fits to experimental data.

\footnotetext{
${ }^{16}$ The problem of calculating power corrections for $W_{\mu \nu}$ with non-convoluted indices is a separate issue which we hope to address in a different publication.
} 
Of course, we made our estimate without taking into account the TMD evolution, notably the most essential double-log (Sudakov) evolution. One should evolve projectile TMD from $\sigma_{a}=\beta_{q}$ to $\tilde{\sigma}_{a}=\frac{q_{\perp}^{2}}{\alpha_{q} s}=\beta_{q} \frac{q_{\perp}^{2}}{Q^{2}}$, target TMDs from $\sigma_{b}=\alpha_{q}$ to $\tilde{\sigma}_{b}=\frac{q_{\perp}^{2}}{\beta_{q} s}=\alpha_{q} \frac{q_{\perp}^{2}}{Q^{2}}$, and match to the result of leading-log calculation of integral over central fields in the rapidity interval between $\tilde{\sigma}_{a}$ and $\tilde{\sigma}_{b}$.

To accurately match these evolutions, we hope to use logic borrowed from the operator product expansion. We write down a general formula (2.14)

$$
W=\frac{1}{(2 \pi)^{4}} \int d^{4} x e^{-i q x} \sum_{m, n} \int d z_{m} c_{m, n}(q, x)\left\langle p_{A}\left|\hat{\Phi}_{A}\left(z_{m}\right)\right| p_{A}\right\rangle \int d z_{n}^{\prime}\left\langle p_{B}\left|\hat{\Phi}_{B}\left(z_{n}^{\prime}\right)\right| p_{B}\right\rangle,
$$

where the coefficient functions $c_{m, n}(q, x)$ are determined by integrals over $C$-fields and do not depend on the form of projectile or target. To find these coefficients in the first-loop order, we integrate over $C$-fields in eq. (2.11) with action $S_{C}=S_{\mathrm{QCD}}\left(C+A+B, \psi_{C}+\right.$ $\left.\psi_{A}+\psi_{B}\right)-S_{\mathrm{QCD}}\left(A, \psi_{A}\right)-S_{\mathrm{QCD}}\left(B, \psi_{B}\right)$ but without any rapidity restrictions on $C$-fields, and subtract matrix elements of the operators $\hat{\Phi}_{A}\left(z_{m}\right) \hat{\Phi}_{B}\left(z_{n}^{\prime}\right)$ in the background fields $A$, $\psi_{A}$ and $B, \psi_{B}$ multiplied by tree-level coefficients. Both the integrals over $C$-fields in eq. (2.11) and matrix elements of $\hat{\Phi}_{A}\left(z_{m}\right) \hat{\Phi}_{B}\left(z_{n}^{\prime}\right)$ will have rapidity divergencies which will be canceled in their sum so what remains are the logarithms (or double logs) of the ratio of kinematical variables ( $Q^{2}$ in our case) to the rapidity cutoffs $\sigma_{a}$ of the operators $\hat{\Phi}_{A}\left(z_{m}\right)$ and $\sigma_{b}$ of $\hat{\Phi}_{B}\left(z_{n}^{\prime}\right)$. Using the above logic we hope to avoid the problem of double-counting of fields which arises when integrals over longitudinal momenta of $C$-fields got pinched at small momenta (see the discussion in the end of secttion 3.2). The work is in progress.

It should be mentioned that, as discussed in ref. [12], our rapidity factorization is different from the standard factorization scheme for particle production in hadron-hadron scattering, namely splitting the diagrams in collinear to projectile part, collinear to target part, hard factor, and soft factor [1]. Here we factorize only in rapidity and the $Q^{2}$ evolution arises from $k_{\perp}^{2}$ dependence of the rapidity evolution kernels, same as in the BK (and NLO BK [36]) equations. Also, since matrix elements of TMD operators with our rapidity cutoffs are UV-finite [37, 38], the only UV divergencies in our approach are usual UV divergencies absorbed in the effective QCD coupling.

It is worth noting that recently the treatment of power corrections was performed in the framework of SCET theory (see e.g. refs. [39-41]). However, since our rapidity factorization is different from factorization used by SCET, the detailed comparison of power corrections to Z-boson (or Higgs) production would be possible when SCET result for TMD corrections in the form of $\frac{1}{m_{Z}^{2}}$ times matrix elements of quark-antiquark-gluon operators will be available.

Let us note that we obtained power corrections for Drell-Yan hadronic tensor convoluted over Lorentz indices. It would be interesting (and we plan) to calculate the highertwist correction to full DY hadronic tensor. Also, it is well known that for semi-inclusive deep inelastic scattering (SIDIS) and for DY process the leading-order TMDs have different directions of Wilson lines: one to $+\infty$ and another to $-\infty[42,43]$. We think that the same directions of Wilson lines will stay on in the case of power corrections and we plan to study this question in forthcoming publications. 


\section{Acknowledgments}

The authors are grateful to S. Dawson, A. Prokudin, T. Rogers, R. Venugopalan, and A. Vladimirov for valuable discussions. This material is based upon work supported by the U.S. Department of Energy, Office of Science, Office of Nuclear Physics under contracts DE-AC02-98CH10886 and DE-AC05-06OR23177 and by U.S. DOE grant DE-FG0297ER41028.

\section{A Next-to-leading quark fields}

In this section we present the explicit expressions for the next-to-leading quark fields $\Psi^{(1)}$. It is convenient to separate these fields in "left" and "right" components:

$$
\Psi^{(1)}=\Psi_{1}^{(1)}+\Psi_{2}^{(1)}, \quad \Psi_{1}^{(1)} \equiv \frac{\not p_{1} \not p_{2}}{s} \Psi^{(1)}, \quad \Psi_{2}^{(1)} \equiv \frac{\not p_{2} \not p_{1}}{s} \Psi^{(1)}
$$

The next-to-leading term in the expansion of the fields (3.19) has the form:

$$
\begin{aligned}
& \Psi_{1 A}^{(1)}=-\frac{g \not p_{1}}{s \beta} \gamma^{i} B_{i} \psi_{A}-\frac{g \gamma^{i}}{s^{2}} \not p_{1} \not p_{2} \frac{1}{\beta} \mathcal{P}_{i} \frac{1}{\alpha} \gamma^{j} B_{j} \psi_{A}-\frac{2 g}{s^{2}} \not p_{1} \not p_{2} \frac{1}{\beta}\left(\mathbb{A}_{\bullet}^{[1]}\right)^{(0)} \psi_{A}, \\
& \Psi_{2 A}^{(1)}=-\frac{2 g \not p_{2} \not p_{1}}{s^{2}} B_{*} \frac{1}{\alpha} \psi_{A}-\frac{g \gamma^{i}}{s^{2}} \not p_{2} \not p_{1} \frac{1}{\alpha} \mathcal{P}_{i} \frac{1}{\beta} \gamma^{j} B_{j} \psi_{A}-\frac{2 g}{s^{2}} \not p_{2} \not p_{1} \frac{1}{\alpha}\left(\mathbb{A}_{*}^{[1]}\right)^{(0)} \psi_{A} \\
& -\frac{2 g^{2} \gamma^{j}}{s^{2} \alpha^{2}} \not p_{2} B_{*} B_{j} \psi_{A}+\frac{g \gamma^{i} \gamma^{j}}{s^{2}} \not p_{2} \frac{1}{\alpha} \mathcal{P}_{i} \frac{1}{\beta} \mathcal{P}_{j} \frac{1}{\alpha} \gamma^{k} B_{k} \psi_{A}, \\
& \Psi_{1 B}^{(1)}=-\frac{g \not p_{2}}{s \alpha} \gamma^{i} A_{i} \psi_{B}-\frac{g \gamma^{i}}{s^{2}} \not p_{2} \not \phi_{1} \frac{1}{\alpha} \mathcal{P}_{i} \frac{1}{\beta} \gamma^{j} A_{j} \psi_{B}-\frac{2 g}{s^{2}} \not p_{2} \not p_{1} \frac{1}{\alpha}\left(\mathbb{A}_{*}^{[1]}\right)^{(0)} \psi_{B}, \\
& \Psi_{2 B}^{(1)}=-\frac{2 g \not p_{1} \not p_{2}}{s^{2}} A_{\bullet} \frac{1}{\beta} \psi_{B}-\frac{g \gamma^{i}}{s^{2}} \not p_{1} \not p_{2} \frac{1}{\beta} \mathcal{P}_{i} \frac{1}{\alpha} \gamma^{j} A_{j} \psi_{B}-\frac{2 g}{s^{2}} \not p_{1} \not p_{2} \frac{1}{\beta}\left(\mathbb{A}_{\bullet}^{[1]}\right)^{(0)} \psi_{B} \\
& -\frac{2 g^{2} \gamma^{j}}{s^{2} \beta^{2}} \not p_{1} A_{\bullet} A_{j} \psi_{B}+\frac{g \gamma^{i} \gamma^{j}}{s^{2}} \not p_{1} \frac{1}{\beta} \mathcal{P}_{i} \frac{1}{\alpha} \mathcal{P}_{j} \frac{1}{\beta} \gamma^{k} A_{k} \psi_{B} \\
& \bar{\Psi}_{1 A}^{(1)}=-\bar{\psi}_{A} \gamma^{i} B_{i} \frac{g \not p_{1}}{s(\beta-i \epsilon)}-\bar{\psi}_{A} \gamma^{j} B_{j} \not p_{2} \not p_{1} \frac{1}{\alpha-i \epsilon} \mathcal{P}_{i} \frac{1}{\beta-i \epsilon} \frac{g \gamma^{i}}{s^{2}}-\frac{2 g}{s^{2}} \bar{\psi}_{A}\left(\mathbb{A}_{\bullet}^{[1]}\right)^{(0)} \frac{1}{\beta-i \epsilon} \not p_{2} \not p_{1} \text {, } \\
& \bar{\Psi}_{2 A}^{(1)}=-\bar{\psi}_{A} \frac{1}{\alpha-i \epsilon} B_{*} \frac{2 g \not p_{1} \not p_{2}}{s^{2}}-\bar{\psi}_{A} \gamma^{j} B_{j} \not p_{1} \not p_{2} \frac{1}{\beta-i \epsilon} \mathcal{P}_{i} \frac{1}{\alpha-i \epsilon} \frac{g \gamma^{i}}{s^{2}}-\frac{2 g}{s^{2}} \bar{\psi}_{A}\left(\mathbb{A}_{*}^{[1]}\right)^{(0)} \frac{1}{\alpha-i \epsilon} \not p_{1} \not p_{2} \\
& -\bar{\psi}_{A} B_{j} B_{*} \not p_{2} \frac{2 g^{2} \gamma^{j}}{s^{2} \alpha^{2}}+\bar{\psi}_{A} \gamma^{k} B_{k} \frac{1}{\alpha-i \epsilon} \mathcal{P}_{j} \frac{1}{\beta-i \epsilon} \mathcal{P}_{i} \frac{1}{\alpha-i \epsilon} \not p_{2} \frac{g \gamma^{j} \gamma^{i}}{s^{2}} \\
& \bar{\Psi}_{1 B}^{(1)}=-\bar{\psi}_{B} \gamma^{i} A_{i} \frac{g \not p_{2}}{s(\alpha-i \epsilon)}-\bar{\psi}_{B} \gamma^{j} A_{j} \not p_{1} \not p_{2} \frac{1}{\beta-i \epsilon} \mathcal{P}_{i} \frac{1}{\alpha-i \epsilon} \frac{g \gamma^{i}}{s^{2}}-\frac{2 g}{s^{2}} \bar{\psi}_{B}\left(\mathbb{A}_{*}^{[1]}\right)^{(0)} \frac{1}{\alpha-i \epsilon} \not p_{1} \not p_{2}, \\
& \bar{\Psi}_{2 B}^{(1)}=-\bar{\psi}_{B} \frac{1}{\beta-i \epsilon} A_{\bullet} \frac{2 g \not p_{2} \not p_{1}}{s^{2}}-\bar{\psi}_{B} \gamma^{j} A_{j} \not p_{2} \not p_{1} \frac{1}{\alpha-i \epsilon} \mathcal{P}_{i} \frac{1}{\beta-i \epsilon} \frac{g \gamma^{i}}{s^{2}}-\frac{2 g}{s^{2}} \bar{\psi}_{B}\left(\mathbb{A}_{\bullet}^{[1]}\right)^{(0)} \frac{1}{\beta-i \epsilon} \not p_{2} \not p_{1} \\
& -\bar{\psi}_{B} A_{j} A_{\bullet} \not p_{1} \frac{2 g^{2} \gamma^{j}}{s^{2} \beta^{2}}+\bar{\psi}_{B} \gamma^{k} A_{k} \frac{1}{\beta} \mathcal{P}_{j} \frac{1}{\alpha} \mathcal{P}_{i} \frac{1}{\beta} \not p_{1} \frac{g \gamma^{j} \gamma^{i}}{s^{2}}
\end{aligned}
$$


where $\mathcal{P}_{i}=i \partial_{i}+g A_{i}+g B_{i}$, see eq. (3.16). The expressions for $\bar{\Psi}$ should be read from right to left, e.g.

$$
\bar{\psi}_{A} \gamma^{j} B_{j} \not p_{2} \not p_{1} \frac{1}{\alpha-i \epsilon} \mathcal{P}_{i} \frac{1}{\beta-i \epsilon}(x) \frac{\gamma^{i}}{s^{2}} \equiv \int d z \bar{\psi}_{A}(z) \gamma^{j} B_{j}(z) \not p_{2} \not p_{1}\left(z\left|\frac{1}{\alpha-i \epsilon} \mathcal{P}_{i} \frac{1}{\beta-i \epsilon}\right| x\right) \frac{\gamma^{i}}{s^{2}}
$$

(and $\frac{1}{\alpha} \equiv \frac{1}{\alpha+i \epsilon} \frac{1}{\beta} \equiv \frac{1}{\beta+i \epsilon}$ as usual). It is easy to see that the power counting of quark fields has the form (cf eq. (3.29)):

$$
\Psi_{1 A}^{(1)} \sim \Psi_{1 B}^{(1)} \sim \Psi_{2 A}^{(1)} \sim \Psi_{2 B}^{(1)} \sim \frac{m_{\perp}^{7 / 2}}{s} .
$$

The gluon fields $\mathbb{A}_{*}^{(0)}$ and $\mathbb{A}_{*}^{(0)}$ were calculated in ref. [12]:

$$
\begin{array}{llrl}
\mathbb{A}_{\bullet}^{(0)} & =A_{\bullet}+\left(\mathbb{A}_{\bullet}^{[1]}\right)^{(0)}, & \left(\mathbb{A}_{\bullet}^{[1] a}\right)^{(0)}=\frac{g}{2 \alpha} A_{j}^{a b} B^{j b}, \\
\mathbb{A}_{*}^{(0)}=B_{*}+\left(\mathbb{A}_{*}^{[1]}\right)^{(0)}, & \left(\mathbb{A}_{*}^{[1] a}\right)^{(0)}=-\frac{g}{2 \beta} A_{j}^{a b} B^{j b}
\end{array}
$$

and their power counting reads

$$
g A_{\bullet} \sim g B_{*} \sim m_{\perp}^{2}, \quad g A_{i} \sim g B_{i} \sim m_{\perp} .
$$

\section{B Formulas with Dirac matrices}

In the gauge $A_{\bullet}=0$ the field $A_{i}$ can be represented as

$$
A_{i}\left(x_{\bullet}, x_{\perp}\right)=\frac{2}{s} \int_{-\infty}^{x} d x_{\bullet}^{\prime} A_{* i}\left(x_{\bullet}^{\prime}, x_{\perp}\right)
$$

(see eq. (3.5)). It is convenient to define a "dual" field

$$
\tilde{A}_{i}\left(x_{\bullet}, x_{\perp}\right)=\frac{2}{s} \int_{-\infty}^{x \bullet} d x_{\bullet}^{\prime} \tilde{A}_{* i}\left(x_{\bullet}^{\prime}, x_{\perp}\right), \quad \tilde{B}_{i}\left(x_{*}, x_{\perp}\right)=\frac{2}{s} \int_{-\infty}^{x_{*}} d x_{*}^{\prime} \tilde{B}_{\bullet i}\left(x_{*}^{\prime}, x_{\perp}\right),
$$

where $\tilde{F}_{\mu \nu}=\frac{1}{2} \epsilon_{\mu \nu \lambda \rho} F^{\lambda \rho}$ as usual. ${ }^{17}$ With this definition, we get

$$
\frac{2}{s} \epsilon_{\bullet * i j} A^{j}=\tilde{A}_{i}, \quad \frac{2}{s} \epsilon_{\bullet * i j} B^{j}=-\tilde{B}_{i} \Rightarrow \tilde{A}_{i} \otimes \tilde{B}^{i}=-A_{i} \otimes B^{i}, \quad \tilde{A}_{i} \otimes B^{i}=A_{i} \otimes \tilde{B}^{i}
$$

and therefore

$$
\begin{aligned}
& A_{k} \gamma_{i} \not p_{2} \gamma^{j} \otimes B_{j} \gamma^{i} \not p_{1} \gamma^{k}=\not p_{2}\left(A_{i}-i \tilde{A}_{i} \gamma_{5}\right) \otimes \not p_{1}\left(B^{i}-i \tilde{B}^{i} \gamma_{5}\right), \\
& A_{k} \gamma^{j} \not p_{2} \gamma_{i} \otimes B_{j} \gamma^{k} \not p_{1} \gamma^{i}=\not p_{2}\left(A_{i}+i \tilde{A}_{i} \gamma_{5}\right) \otimes \not p_{1}\left(B^{i}+i \tilde{B}^{i} \gamma_{5}\right), \\
& A_{k} \gamma_{i} \not p_{2} \gamma_{j} \otimes B^{j} \gamma^{k} \not p_{1} \gamma^{i}=\not p_{2}\left(A_{i}-i \tilde{A}_{i} \gamma_{5}\right) \otimes \not p_{1}\left(B^{i}+i \tilde{B}^{i} \gamma_{5}\right), \\
& A_{k} \gamma_{j} \not \not_{2} \gamma_{i} \otimes B^{j} \gamma^{i} \not p_{1} \gamma^{k}=\not p_{2}\left(A_{i}+i \tilde{A}_{i} \gamma_{5}\right) \otimes \not p_{1}\left(B^{i}-i \tilde{B}^{i} \gamma_{5}\right) .
\end{aligned}
$$

\footnotetext{
${ }^{17}$ We use conventions from Bjorken ${ }^{6}$ D Dell where $\epsilon^{0123}=-1$ and $\gamma^{\mu} \gamma^{\nu} \gamma^{\lambda}=g^{\mu \nu} \gamma^{\lambda}+g^{\nu \lambda} \gamma^{\mu}-g^{\mu \lambda} \gamma^{\nu}-$ $i \epsilon^{\mu \nu \lambda \rho} \gamma_{\rho} \gamma_{5}$. Also, with this convention $\tilde{\sigma}_{\mu \nu} \equiv \frac{1}{2} \epsilon_{\mu \nu \lambda \rho} \sigma^{\lambda \rho}=i \sigma_{\mu \nu} \gamma_{5}$.
} 
We will also need

$$
\begin{aligned}
& A_{k} \gamma_{i} \not p_{2} \gamma^{j} \gamma_{5} \otimes B_{j} \gamma^{i} \not p_{1} \gamma^{k} \gamma_{5}=A_{k} \gamma_{i} \not p_{2} \gamma^{j} \otimes B_{j} \gamma^{i} \not p_{1} \gamma^{k}, \\
& A_{k} \gamma^{j} \not p_{2} \gamma_{i} \gamma_{5} \otimes B_{j} \gamma^{k} \not p_{1} \gamma^{i} \gamma_{5}=A_{k} \gamma^{j} \not p_{2} \gamma_{i} \otimes B_{j} \gamma^{k} \not p_{1} \gamma^{i}, \\
& A_{k} \gamma_{i} \not p_{2} \gamma_{j} \gamma_{5} \otimes B^{j} \gamma^{k} \not p_{1} \gamma^{i} \gamma_{5}=-A_{k} \gamma_{i} \not p_{2} \gamma_{j} \otimes B^{j} \gamma^{k} \not p_{1} \gamma^{i}, \\
& A_{k} \gamma_{j} \not p_{2} \gamma_{i} \gamma_{5} \otimes B^{j} \gamma^{i} \not p_{1} \gamma^{k} \gamma_{5}=-A_{k} \gamma_{j} \not p_{2} \gamma_{i} \otimes B^{j} \gamma^{i} \not p_{1} \gamma^{k}
\end{aligned}
$$

and hence

$$
\begin{aligned}
& A_{k} \gamma_{i} \not p_{2} \gamma^{j}\left(a-\gamma_{5}\right) \otimes B_{j} \gamma^{i} \not p_{1} \gamma^{k}\left(a-\gamma_{5}\right) \\
& \quad=\left(a^{2}+1\right) \not p_{2}\left(A_{i}-i \tilde{A}_{i} \gamma_{5}\right) \otimes \not p_{1}\left(B^{i}-i \tilde{B}^{i} \gamma_{5}\right)-2 a \not p_{2}\left(A_{i}-i \tilde{A}_{i} \gamma_{5}\right) \otimes \not p_{1}\left(\gamma_{5} B^{i}-i \tilde{B}^{i}\right), \\
& A_{k} \gamma^{j} \not p_{2} \gamma_{i}\left(a-\gamma_{5}\right) \otimes B_{j} \gamma^{k} \not p_{1} \gamma^{i}\left(a-\gamma_{5}\right) \\
& \quad=\left(a^{2}+1\right) \not p_{2}\left(A_{i}+i \tilde{A}_{i} \gamma_{5}\right) \otimes \not p_{1}\left(B^{i}+i \tilde{B}^{i} \gamma_{5}\right)-2 a \not p_{2}\left(A_{i}+i \tilde{A}_{i} \gamma_{5}\right) \otimes \not p_{1}\left(\gamma_{5} B^{i}+i \tilde{B}^{i}\right), \\
& A_{k} \gamma_{i} \not p_{2} \gamma_{j}\left(a-\gamma_{5}\right) \otimes B^{j} \gamma^{k} \not p_{1} \gamma^{i}\left(a-\gamma_{5}\right)=\left(a^{2}-1\right) \not p_{2}\left(A_{i}-i \tilde{A}_{i} \gamma_{5}\right) \otimes \not p_{1}\left(B^{i}+i \tilde{B}^{i} \gamma_{5}\right), \\
& A_{k} \gamma_{j} \not p_{2} \gamma_{i}\left(a-\gamma_{5}\right) \otimes B^{j} \gamma^{i} \not p_{1} \gamma^{k}\left(a-\gamma_{5}\right)=\left(a^{2}-1\right) \not p_{2}\left(A_{i}+i \tilde{A}_{i} \gamma_{5}\right) \otimes \not p_{1}\left(B^{i}-i \tilde{B}^{i} \gamma_{5}\right) .
\end{aligned}
$$

Next, using formula

$$
\begin{aligned}
\tilde{\sigma}_{\mu \nu} \otimes \tilde{\sigma}_{\alpha \beta}= & -\frac{1}{2}\left(g_{\mu \alpha} g_{\nu \beta}-g_{\nu \alpha} g_{\mu \beta}\right) \sigma_{\xi \eta} \otimes \sigma^{\xi \eta} \\
& +g_{\mu \alpha} \sigma_{\beta \xi} \otimes \sigma_{\nu}^{\xi}-g_{\nu \alpha} \sigma_{\beta \xi} \otimes \sigma_{\mu}^{\xi}-g_{\mu \beta} \sigma_{\alpha \xi} \otimes \sigma_{\nu}^{\xi}+g_{\nu \beta} \sigma_{\alpha \xi} \otimes \sigma_{\mu}^{\xi}-\sigma_{\alpha \beta} \otimes \sigma_{\mu \nu}
\end{aligned}
$$

we get

$$
\begin{aligned}
& A^{k} \not p_{2} \gamma_{j} \otimes B^{j} \not p_{1} \gamma_{k}-A^{k} \not p_{2} \gamma_{j} \gamma_{5} \otimes B^{j} \not p_{1} \gamma_{k} \gamma_{5} \\
& =A^{k} \not p_{2} \gamma_{j} \otimes B^{j} \not p_{1} \gamma_{k}+A^{k} \not p_{2} \gamma_{k} \otimes B^{j} \not p_{1} \gamma_{j}-\frac{s}{4} A^{j} \sigma_{\xi \eta} \otimes B_{j} \sigma^{\xi \eta}-\frac{s}{2} A^{k} \sigma_{k i} \otimes B^{j} \sigma_{j}{ }^{i} \\
& \quad-A^{j} \not p_{1} \gamma_{k} \otimes B_{j} \not p_{2} \gamma^{k}+\frac{s}{2} A^{i} \sigma_{j k} \otimes B_{i} \sigma^{j k}-\frac{2}{s} A^{k} \sigma_{* \bullet} \otimes B_{k} \sigma_{* \bullet}-2 A^{k} \not p_{2} \gamma_{j} \otimes B_{k} \not p_{1} \gamma^{j}
\end{aligned}
$$

For appendix $\mathrm{C}$ we also need

$$
\begin{aligned}
\frac{2}{s}\left(\hat{p}_{2} \gamma^{i} \hat{p}_{1} \otimes \hat{p}_{1} B_{i}+\hat{p}_{2} \gamma^{i} \hat{p}_{1} \gamma_{5} \otimes \hat{p}_{1} \gamma_{5} B_{i}\right) & =-\gamma^{i} \otimes \hat{p}_{1}\left(B_{i}+i \tilde{B}_{i} \gamma_{5}\right)-\gamma^{i} \gamma_{5} \otimes \hat{p}_{1} \gamma_{5}\left(B_{i}+i \tilde{B}_{i} \gamma_{5}\right), \\
\gamma_{k} \gamma^{i} \hat{p}_{2} \otimes B_{i} \gamma^{k}+\gamma_{k} \gamma^{i} \hat{p}_{2} \gamma_{5} \otimes B_{i} \gamma^{k} \gamma_{5} & =\hat{p}_{2} \otimes\left(B_{i}+i \tilde{B}_{i} \gamma_{5}\right) \gamma^{i}+\hat{p}_{2} \gamma_{5} \otimes\left(B_{i}+i \tilde{B}_{i} \gamma_{5}\right) \gamma^{i} \gamma_{5}, \\
\not p_{2} \gamma^{i} \gamma^{j} \otimes \not p_{1} B_{j}+\not p_{2} \gamma_{5} \gamma^{i} \gamma^{j} \otimes \not p_{1} \gamma_{5} B_{j} & =\not p_{2} \otimes\left(B^{i}+i \tilde{B}^{i} \gamma_{5}\right) \not p_{1}+\gamma_{5} \not p_{2} \otimes \gamma_{5}\left(B^{i}+i \tilde{B}^{i} \gamma_{5}\right) \not p_{1}, \\
\not p_{2} \gamma^{i} \gamma^{j} \otimes \not p_{1} B_{j}+\not p_{2} \gamma_{5} \gamma^{i} \gamma^{j} \otimes \not p_{1} \gamma_{5} B_{j} & =\not p_{2} \otimes \not p_{1}\left(B^{i}-i \tilde{B}^{i} \gamma_{5}\right)+\not p_{2} \gamma_{5} \otimes \not p_{1} \gamma_{5}\left(B^{i}-i \tilde{B}^{i} \gamma_{5}\right) .
\end{aligned}
$$

\section{Subleading power corrections}

\section{C.1 Second, third, and fourth lines in eq. (4.19)}

In this appendix we show that second, third, and fourth lines in eq. (4.19) yield subleading power corrections and can be neglected in our approximation. 
Let us consider for example the last term in the third line of eq. (4.19). The Fierz transformation (4.8) yields

$$
\begin{aligned}
& {\left[\bar{\psi}_{A}(x) \breve{\gamma}_{\mu} \psi_{B}(x)\right]\left[\bar{\psi}_{B}(0) \breve{\gamma}^{\mu} \Xi_{2 A}(0)\right] } \\
&=\frac{1+a^{2}}{2}\left\{\left[\bar{\psi}_{A}^{m}(x) \gamma_{\alpha} \Xi_{2 A}^{n}(0)\right]\left[\bar{\psi}_{B}^{n}(0) \gamma^{\alpha} \psi_{B}^{m}(x)\right]+\left(\gamma_{\alpha} \otimes \gamma^{\alpha} \leftrightarrow \gamma_{\alpha} \gamma_{5} \otimes \gamma^{\alpha} \gamma_{5}\right)\right\} \\
&-a\left\{\left[\bar{\psi}_{A}^{m}(x) \gamma_{\alpha} \Xi_{2 A}^{n}(0)\right]\left[\bar{\psi}_{B}^{n}(0) \gamma^{\alpha} \gamma_{5} \psi_{B}^{m}(x)\right]+\left(\gamma_{\alpha} \otimes \gamma^{\alpha} \gamma_{5} \leftrightarrow \gamma_{\alpha} \gamma_{5} \otimes \gamma^{\alpha}\right)\right\} \\
&+\left(1-a^{2}\right)\left\{\left[\bar{\psi}_{A}^{m}(x) \Xi_{2 A}^{n}(0)\right]\left[\bar{\psi}_{B}^{n}(0) \psi_{B}^{m}(x)\right]-\left(1 \otimes 1 \leftrightarrow \gamma_{5} \otimes \gamma_{5}\right)\right\} \\
&= \frac{1+a^{2}}{2}\left\{\left[\bar{\psi}_{A}^{m}(x) \gamma_{i} \Xi_{2 A}^{n}(0)\right]\left[\bar{\psi}_{B}^{n}(0) \gamma^{i} \psi_{B}^{m}(x)\right]+\left(\gamma_{i} \otimes \gamma^{i} \leftrightarrow \gamma_{i} \gamma_{5} \otimes \gamma^{i} \gamma_{5}\right)\right\} \\
&-a\left\{\left[\bar{\psi}_{A}^{m}(x) \gamma_{i} \Xi_{2 A}^{n}(0)\right]\left[\bar{\psi}_{B}^{n}(0) \gamma^{i} \gamma_{5} \psi_{B}^{m}(x)\right]+\left(\gamma_{i} \otimes \gamma^{i} \gamma_{5} \leftrightarrow \gamma_{i} \gamma_{5} \otimes \gamma^{i}\right)\right\} \\
&+\left(1-a^{2}\right)\left\{\left[\bar{\psi}_{A}^{m}(x) \Xi_{2 A}^{n}(0)\right]\left[\bar{\psi}_{B}^{n}(0) \psi_{B}^{m}(x)\right]-\left(1 \otimes 1 \leftrightarrow \gamma_{5} \otimes \gamma_{5}\right)\right\}+O\left(\frac{m_{\perp}^{8}}{s}\right) .
\end{aligned}
$$

Sorting out the color-singlet contributions ${ }^{18}$ we get

$$
\begin{aligned}
\left\langle A, B\left|\left(\bar{\psi}_{A}^{m}\left(B_{j}\right)^{n k} \psi_{A}^{k}\right)\left(\bar{\psi}_{B}^{n} \psi_{B}^{m}\right)\right| A, B\right\rangle & =\left\langle A, B\left|\left(\bar{\psi}_{A}^{m} \psi_{A}^{k}\right)\left(\bar{\psi}_{B}^{n}\left(B_{j}\right)^{n k} \psi_{B}^{m}\right)\right| A, B\right\rangle \\
& =\frac{1}{N_{c}}\left\langle A\left|\left(\bar{\psi}_{A}^{l} \psi_{A}^{l}\right)\right| A\right\rangle\left\langle B\left|\left(\bar{\psi}_{B}^{n} B_{j}^{n k} \psi_{B}^{k}\right)\right| B\right\rangle
\end{aligned}
$$

and therefore

$$
\begin{aligned}
- & N_{c}\left[\bar{\psi}_{A}(x) \breve{\gamma}_{\mu} \psi_{B}(x)\right]\left[\bar{\psi}_{B}(0) \breve{\gamma}^{\mu} \Xi_{2 A}(0)\right] \\
= & \frac{1+a^{2}}{2 s}\left\{\left[\bar{\psi}_{A}(x) \gamma_{i} \not p_{2} \gamma^{j} \frac{1}{\alpha} \psi_{A}(0)\right]\left[\bar{\psi}_{B}(0) g B_{j}(0) \gamma^{i} \psi_{B}(x)\right]+\left(\gamma_{i} \otimes \gamma^{i} \leftrightarrow \gamma_{i} \gamma_{5} \otimes \gamma^{i} \gamma_{5}\right)\right\} \\
& +\frac{1-a^{2}}{s}\left\{\left[\bar{\psi}_{A}(x) \not p_{2} \gamma^{j} \frac{1}{\alpha} \psi_{A}(0)\right]\left[\bar{\psi}_{B}(0) g B_{j}(0) \psi_{B}(x)\right]-\left(1 \otimes 1 \leftrightarrow \gamma_{5} \otimes \gamma_{5}\right)\right\} \\
& -\frac{a}{s}\left\{\left[\bar{\psi}_{A}(x) \gamma_{i} \gamma_{5} \not p_{2} \gamma^{j} \frac{1}{\alpha} \psi_{A}(0)\right]\left[\bar{\psi}_{B}(0) g B_{j}(0) \gamma^{i} \psi_{B}(x)\right]+\left(\gamma_{i} \gamma_{5} \otimes \gamma^{i} \leftrightarrow \gamma_{i} \otimes \gamma^{i} \gamma_{5}\right)\right\},
\end{aligned}
$$

where $\frac{1}{\alpha} \equiv \frac{1}{\alpha+i \epsilon}$, see eq. (3.28).

For the forward matrix elements we get

$$
\begin{aligned}
& \frac{1}{s} \int d x_{\perp} d x_{\bullet} e^{-i \alpha_{q} x_{\bullet}+i(k, x)_{\perp}}\left\langle A\left|\hat{\bar{\psi}}\left(x_{\bullet}, x_{\perp}\right) p_{2} \Gamma \frac{1}{\alpha} \hat{\psi}(0)\right| A\right\rangle \\
& =\frac{1}{\alpha_{q} s} \int d x_{\perp} d x_{\bullet} e^{-i \alpha_{q} x_{\bullet}+i(k, x)_{\perp}}\left\langle A\left|\hat{\bar{\psi}}\left(x_{\bullet}, x_{\perp}\right) \not p_{2} \Gamma \hat{\psi}(0)\right| A\right\rangle \sim \frac{1}{\alpha_{q}} f_{\Gamma}\left(\alpha_{q}, k_{\perp}^{2}\right),
\end{aligned}
$$

where $\Gamma$ is any of $\gamma$-matrices with transverse indices. Next, consider

$$
\begin{aligned}
& \int d x_{\perp} d x_{*} e^{-i \beta_{q} x_{*}+i(k, x)_{\perp}}\left\langle B\left|\hat{\bar{\psi}}(0) g \hat{B}_{i}(0) \hat{\psi}\left(x_{*}, x_{\perp}\right)\right| B\right\rangle \\
& =\frac{2}{s} \int d x_{\perp} d x_{*} e^{-i \beta_{q} x_{*}+i(k, x)_{\perp}} \int_{-\infty}^{0} d x_{*}^{\prime}\left\langle B\left|\hat{\bar{\psi}}(0) g \hat{F}_{\bullet i}\left(x_{*}^{\prime}, 0_{\perp}\right) \hat{\psi}\left(x_{*}, x_{\perp}\right)\right| B\right\rangle \sim \frac{k_{i}}{m} f^{\mathrm{tw} 3}\left(\beta_{q}, k_{\perp}^{2}\right),
\end{aligned}
$$

\footnotetext{
${ }^{18}$ Recall that after the promotion of background fields to operators we can still move those operators freely since all of them commute, see footnotes 2,10 and 11.
} 
where $f^{\text {tw3 }}\left(\beta_{q}, k_{\perp}^{2}\right)$ is some function of order one (by power counting (3.4) this matrix element (C.5) is $\sim 1$ ). Also, this function may have only logarithmical singularities in $\beta_{q}$ as $\beta_{q} \rightarrow 0$ but not the power behavior $\frac{1}{\beta_{q}} \cdot{ }^{19}$ The corresponding contribution to $W(q)$ of eq. (4.1) is proportional to

$$
\frac{1}{\alpha_{q} s} \int d^{2} k_{\perp} f_{\Gamma_{i}}\left(\alpha_{q}, k_{\perp}\right) k_{i} f^{\mathrm{tw} 3}\left(\beta_{q}, k_{\perp}^{2}\right) \sim \frac{q_{\perp}^{2}}{\alpha_{q} s} W^{\mathrm{lt}} \ll \frac{q_{\perp}^{2}}{Q^{2}} W^{\mathrm{lt}}
$$

so it can be neglected in comparison to the contributions $\sim \frac{q_{\perp}^{2}}{Q^{2}} W^{\text {lt }}$ (recall that we assume that Z-boson is produced in a central range of rapidity so $\left.\frac{q_{\perp}^{2}}{\alpha_{q} s} \simeq \frac{q_{\perp}^{2}}{Q \sqrt{s}} \ll \frac{q_{\perp}^{2}}{Q^{2}}\right)$. In a similar way one can show that the remaining three terms in the second and third lines of eq. (4.19) give small contributions to $W(q)$.

Next, it is easy to see that the matrix element of the fourth line of eq. (4.19) vanishes. Indeed, let us consider the first term in the fourth line and perform Fierz transformation (4.8):

$$
\begin{aligned}
& {\left[\bar{\Xi}_{2 A}(x) \breve{\gamma}_{\mu} \psi_{B}(x)\right]\left[\bar{\psi}_{B}(0) \breve{\gamma}^{\mu} \Xi_{2 A}(0)\right]} \\
& =\frac{1+a^{2}}{2}\left\{\left[\bar{\Xi}_{2 A}^{m}(x) \gamma_{\alpha} \Xi_{2 A}^{n}(0)\right]\left[\bar{\psi}_{B}^{n}(0) \gamma^{\alpha} \psi_{B}^{m}(x)\right]+\left(\gamma_{\alpha} \otimes \gamma^{\alpha} \leftrightarrow \gamma_{\alpha} \gamma_{5} \otimes \gamma^{\alpha} \gamma_{5}\right)\right\} \\
& \quad-a\left(\left[\bar{\Xi}_{2 A}^{m}(x) \gamma_{\alpha} \Xi_{2 A}^{n}(0)\right]\left[\bar{\psi}_{B}^{n}(0) \gamma^{\alpha} \gamma_{5} \psi_{B}^{m}(x)\right]+\left(\gamma_{\alpha} \otimes \gamma^{\alpha} \gamma_{5} \leftrightarrow \gamma_{\alpha} \gamma_{5} \otimes \gamma^{\alpha}\right)\right\} \\
& \quad+\left(1-a^{2}\right)\left(\left[\bar{\Xi}_{2 A}^{m}(x) \Xi_{2 A}^{n}(0)\right]\left[\bar{\psi}_{B}^{n}(0) \psi_{B}^{m}(x)\right]-\left(1 \otimes 1 \leftrightarrow \gamma_{5} \otimes \gamma_{5}\right)\right\} .
\end{aligned}
$$

From the explicit form of $\Xi_{2 A}$ and $\bar{\Xi}_{2 A}$ in eq. (3.27) we see that the last term in the r.h.s. vanishes while the first two are small. Indeed,

$$
\begin{aligned}
& \left\langle A, B\left|\frac{2}{s}\left[\bar{\Xi}_{2 A}^{m}(x) \not p_{1} \Xi_{2 A}^{n}(0)\right]\left[\bar{\psi}_{B}^{n}(0) \not p_{2} \psi_{B}^{m}(x)\right]\right| A, B\right\rangle \\
& =\left\langle A, B\left|\frac{2}{s}\left[\left(\bar{\psi}_{A}^{k} \frac{1}{\alpha}\right)(x) \gamma^{i} \frac{\not p_{2}}{s} \gamma^{j} \frac{1}{\alpha} \psi_{A}^{l}(0)\right]\left[\bar{\psi}_{B}^{n}(0) g^{2} \hat{B}_{i}^{k m}(x) \hat{B}_{j}^{n l}(0) \not p_{2} \psi_{B}^{m}(x)\right]\right| A, B\right\rangle \\
& =\frac{2}{s N_{c}}\left\langle A\left|\left(\hat{\bar{\psi}} \frac{1}{\alpha}\right)(x) \gamma^{i} \frac{\not p_{2}}{s} \gamma^{j} \frac{1}{\alpha} \hat{\psi}(0)\right| A\right\rangle\left\langle B\left|\hat{\bar{\psi}}(0) g^{2} \hat{A}_{j}(0) \hat{A}_{i}(x) \not p_{2} \hat{\psi}(x)\right| B\right\rangle \sim O\left(\frac{m_{\perp}^{8}}{s}\right),
\end{aligned}
$$

so the contribution to $W$ is of order of $\frac{m_{1}^{4}}{s^{2}} W^{\mathrm{lt}}$.

\section{C.2 Second to fifth lines in eq. (4.56)}

Here we show that second to fifth lines in eq. (4.56) either vanish or can be neglected.

Obviously, matrix element of the operator in the second line vanishes. Formally,

$$
\begin{aligned}
& \int d x_{\bullet} e^{-i \alpha_{q} x_{\bullet}}\left\langle A\left|\hat{\bar{\psi}}\left(x_{\bullet}, x_{\perp}\right) \breve{\gamma}_{\mu} \hat{\psi}\left(x_{\bullet}, x_{\perp}\right)\right| A\right\rangle=\delta\left(\alpha_{q}\right)\left\langle A\left|\hat{\bar{\psi}}(0) \breve{\gamma}_{\mu} \hat{\psi}(0)\right| A\right\rangle, \\
& \int d x_{*} e^{-i \beta_{q} x_{*}}\left\langle B\left|\hat{\bar{\psi}}(0) \breve{\gamma} \breve{\gamma}_{\mu} \hat{\psi}(0)\right| B\right\rangle=\delta\left(\beta_{q}\right)\left\langle B\left|\hat{\bar{\psi}}(0) \breve{\gamma}_{\mu} \hat{\psi}(0)\right| B\right\rangle
\end{aligned}
$$

\footnotetext{
${ }^{19}$ Large $x_{*}$ correspond to low- $x$ domain where matrix elements can be calculated in a shock-wave background of the target particle. The typical propagator in the shock-wave external field has a factor $e^{-i \frac{p_{\perp}^{2}}{\alpha s}(x-z)_{*}}$ where $z_{*}$ is a position of a shock wave and $p_{\perp}$ is of order of characteristic transverse momentum $[44,45]$. The integration over large $x_{*}$ gives then $\left(\beta_{q}+\frac{p_{\perp}^{2}}{\alpha s}\right)^{-1}$ and since the integration over $\alpha$ is restricted from above by $\sigma_{a}$, such terms cannot give $\frac{1}{\beta_{q}}$ (cf. refs $[37,38]$ ).
} 
and, non-formally, one hadron cannot produce Z-boson on his own. For a similar reason, matrix elements of the operators in the third and fourth lines in eq. (4.56) vanish - either projectile or target matrix element will be of eq. (C.9) type. In addition, from the explicit form $\Xi$ 's in eq. (3.27) it is easy to see that the fifth line in eq. (4.56) can be rewritten as follows:

$$
\begin{aligned}
& {\left[\bar{\Xi}_{2 A}(x) \breve{\gamma}_{\mu} \Xi_{2 A}(x)\right]\left[\bar{\psi}_{B}(0) \breve{\gamma}^{\mu} \psi_{B}(0)\right]+\left[\bar{\psi}_{A}(x) \breve{\gamma}_{\mu} \psi_{A}(x)\right]\left[\bar{\Xi}_{1 B}(0) \breve{\gamma}^{\mu} \Xi_{1 B}(0)\right]+x \leftrightarrow 0} \\
& =\left[\left(\bar{\psi}_{A} \frac{1}{\alpha}\right)(x) \gamma^{i} g B_{i}(x) \frac{\not p_{2}}{s} \breve{\gamma}_{\mu} \frac{\not p_{2}}{s} \gamma^{k} g B_{k}(x) \frac{1}{\alpha} \psi_{A}(x)\right]\left[\bar{\psi}_{B}(0) \breve{\gamma}^{\mu} \psi_{B}(0)\right] \\
& \quad+\left[\bar{\psi}_{A}(x) \breve{\gamma}_{\mu} \psi_{A}(x)\right]\left[\left(\bar{\psi}_{B} \frac{1}{\beta}\right)(0) \gamma^{i} g A_{i}(0) \frac{\not p_{1}}{s} \breve{\gamma}^{\mu} \frac{\not p_{1}}{s} \gamma^{k} g A_{k}(0) \frac{1}{\beta} \psi_{B}(0)\right]+x \leftrightarrow 0 \\
& =2\left[\left(\bar{\psi}_{A} \frac{1}{\alpha}\right)(x) \gamma^{i} g B_{i}(x) \frac{\not p_{2}}{s^{2}}\left(a-\gamma_{5}\right) \gamma^{k} g B_{k}(x) \frac{1}{\alpha} \psi_{A}(x)\right]\left[\bar{\psi}_{B}(0) \not p_{2}\left(a-\gamma_{5}\right) \psi_{B}(0)\right] \\
& \quad+2\left[\bar{\psi}_{A}(x) \not p_{1}\left(a-\gamma_{5}\right) \psi_{A}(x)\right]\left[\left(\bar{\psi}_{B} \frac{1}{\beta}\right)(0) \gamma^{i} g A_{i}(0) \frac{\not p_{1}}{s^{2}}\left(a-\gamma_{5}\right) \gamma^{k} g A_{k}(0) \frac{1}{\beta} \psi_{B}(0)\right]+x \leftrightarrow 0 .
\end{aligned}
$$

From the power counting (3.4) we see that this term is $\sim \frac{m_{\perp}^{8}}{s}$, so we are left with the contribution of the last two lines in eq. (4.56).

\section{C.3 Gluon power corrections from $\mathcal{J}_{A}^{\mu}(x) \mathcal{J}_{A \mu}(0)$ terms}

There is one more contribution which should be discussed and neglected:

$$
\begin{aligned}
& \mathcal{J}_{A}^{\mu}(x) \mathcal{J}_{A \mu}(0)=\frac{e^{2}}{16 s_{W}^{2} c_{W}^{2}} \\
& \quad \times\left[\left(\bar{\psi}_{A}(x)+\bar{\Xi}_{2 A}(x)\right) \breve{\gamma}_{\mu}\left(\psi_{A}(x)+\Xi_{2 A}(x)\right)\right]\left[\left(\bar{\psi}_{A}(0)+\bar{\Xi}_{2 A}(0)\right) \breve{\gamma}^{\mu}\left(\psi_{A}(0)+\Xi_{2 A}(0)\right)\right] \\
& =\frac{e^{2}}{16 s_{W}^{2} c_{W}^{2}}\left(\left[\bar{\Xi}_{2 A}(x) \breve{\gamma}_{\mu} \psi_{A}(x)\right]\left[\bar{\psi}_{A}(0) \breve{\gamma}^{\mu} \Xi_{2 A}(0)\right]+\left[\bar{\psi}_{A}(x) \breve{\gamma}_{\mu} \Xi_{2 A}(x)\right]\left[\bar{\Xi}_{2 A}(0) \breve{\gamma}^{\mu} \psi_{A}(0)\right]\right. \\
& \left.\quad+\left[\bar{\Xi}_{2 A}(x) \breve{\gamma}_{\mu} \psi_{A}(x)\right]\left[\bar{\Xi}_{2 A}(0) \breve{\gamma}^{\mu} \psi_{A}(0)\right]+\left[\bar{\psi}_{A}(x) \breve{\gamma}_{\mu} \Xi_{2 A}(x)\right]\left[\bar{\psi}_{A}(0) \breve{\gamma}^{\mu} \Xi_{2 A}(0)\right]\right), \quad \text { C.1 }
\end{aligned}
$$

where we neglected terms which cannot contribute to $W$ due to the reason discussed after eq. (C.9), i.e. that one hadron ("A" or "B") cannot produce Z-boson on its own.

Let us consider the first term in the r.h.s. of this equation

$$
\begin{aligned}
& {\left[\Xi_{2 A}(x) \breve{\gamma}_{\mu} \psi_{A}(x)\right]\left[\bar{\psi}_{A}(0) \breve{\gamma}^{\mu} \Xi_{2 A}(0)\right]} \\
& \stackrel{g^{2}}{\rightarrow}-\frac{p^{2}}{2 N_{c}\left(N_{c}^{2}-1\right)}\left\langle A\left|\left(\hat{\bar{\psi}} \frac{1}{\alpha}\right)(x) \gamma_{i} \frac{p_{2}}{s} \breve{\gamma}_{\mu} \hat{\psi}(x) \hat{\bar{\psi}}(0) \breve{\gamma}^{\mu} \frac{\not p_{2}}{s} \gamma_{j} \frac{1}{\alpha} \hat{\psi}(0)\right| A\right\rangle\left\langle B\left|\hat{A}^{a i}(x) \hat{A}^{a j}(0)\right| B\right\rangle \\
& =-\frac{g^{2}}{2 N_{c}\left(N_{c}^{2}-1\right)}\left\langle A\left|\left(\hat{\bar{\psi}} \frac{1}{\alpha}\right)(x) \gamma_{i} \frac{\not p_{2}}{s} \breve{\gamma}_{k} \hat{\psi}(x) \hat{\bar{\psi}}(0) \breve{\gamma}^{k} \frac{\not p_{2}}{s} \gamma_{j} \frac{1}{\alpha} \hat{\psi}(0)\right| A\right\rangle \\
& \times\left\langle B\left|\hat{A}^{a i}(x) \hat{A}^{a j}(0)\right| B\right\rangle+O\left(\frac{m_{\perp}^{8}}{s}\right),
\end{aligned}
$$

where $\stackrel{\text { f.int. }}{\rightarrow}$ denotes functional integration over $A$ and $B$ fields in eq. (2.8).

The matrix element $\left\langle B\left|\hat{A}^{a i}(x) \hat{A}^{a j}(0)\right| B\right\rangle$ for unpolarized hadrons can be proportional either to $2 x^{i} x^{j}+x_{\perp}^{2} g^{i j}$ or to $g^{i j}$. Since the former structure does not contribute due to

$$
\left(2 x^{i} x^{j}+x_{\perp}^{2} g^{i j}\right) \gamma_{i} \not p_{2} \gamma^{k} \otimes \gamma_{k} \not p_{2} \gamma_{j}=0
$$


we get

$$
\begin{aligned}
& \left\langle A, B\left|\left[\bar{\Xi}_{2 A}(x) \breve{\gamma}_{\mu} \psi_{A}(x)\right]\left[\bar{\psi}_{A}(0) \breve{\gamma}^{\mu} \Xi_{2 A}(0)\right]\right| A, B\right\rangle \\
& =-\frac{g^{2}}{2 N_{c}\left(N_{c}^{2}-1\right) s^{2}}\left\langle A\left|\hat{\bar{\psi}} \frac{1}{\alpha}(x) \not p_{2}\left(a-\gamma_{5}\right) \hat{\psi}(x) \hat{\bar{\psi}}(0) \not p_{2}\left(a-\gamma_{5}\right) \frac{1}{\alpha} \hat{\psi}(0)\right| A\right\rangle \\
& \quad \times\left\langle B\left|\hat{A}_{i}^{a}(x) \hat{A}^{a i}(0)\right| B\right\rangle+O\left(\frac{m_{\perp}^{8}}{s}\right) .
\end{aligned}
$$

For the forward target matrix element one obtains

$$
\begin{aligned}
\int d x_{*} & e^{-i \beta_{q} x_{*}}\left\langle B\left|\hat{A}_{i}^{a}(x) \hat{A}^{a i}(0)\right| B\right\rangle \\
& =\frac{4}{s^{2}} \int d x_{*} e^{-i \beta_{q} x_{*}} \int_{-\infty}^{x_{*}} d x_{*}^{\prime} \int_{-\infty}^{0} d x_{*}^{\prime \prime}\left\langle B\left|\hat{F}_{\bullet i}^{a}\left(x_{*}^{\prime}, x_{\perp}\right) \hat{F}_{\bullet}^{a i}\left(x_{*}^{\prime \prime}, 0_{\perp}\right)\right| B\right\rangle \\
& =\frac{4}{\beta_{q}^{2} s^{2}} \int d x_{*} e^{-i \beta_{q} x_{*}}\left\langle B\left|\hat{F}_{\bullet i}^{a}\left(x_{*}, x_{\perp}\right) \hat{F}_{\bullet}^{a i}(0)\right| B\right\rangle=-\frac{1}{\beta_{q}} 8 \pi^{2} \alpha_{s} \mathcal{D}_{g}\left(\beta_{q}, x_{\perp}\right),
\end{aligned}
$$

where we used parametrization (4.6) from ref. [12]. Since the gluon TMD $\mathcal{D}_{g}\left(x_{B}, x_{\perp}\right)$ behaves only logarithmically as $x_{B} \rightarrow 0$ [38], the contribution of eq. (C.14) to $W(q)$ is of order of $\frac{m_{\perp}^{2}}{\beta_{q} s} \ll \frac{m_{\perp}^{2}}{Q^{2}}$ (note that the projectile TMD in the r.h.s. of eq. (C.12) does not have $\frac{1}{\alpha_{q}}$ terms for the same reason as in eq. (C.22)). Similarly, all other terms in eq. (C.11) are either $\frac{m_{\perp}^{2}}{\beta_{q} s}$ or $\frac{m_{\perp}^{2}}{\alpha_{q} s}$ so they can be neglected. ${ }^{20}$

\section{C.4 Power corrections from $\Psi^{(1)}$ fields}

First, let us notice that terms like

$$
\bar{\psi}_{A}(x) \breve{\gamma}_{\mu} \psi_{A}(x) \bar{\psi}_{A}(0) \breve{\gamma}^{\mu} \Psi_{A}^{(1)}(0), \quad \bar{\psi}_{A}(x) \breve{\gamma}_{\mu} \psi_{A}(x) \bar{\psi}_{B}(0) \breve{\gamma}^{\mu} \Psi_{B}^{(1)}(0)
$$

give zero contribution since $\bar{\psi}_{A}(x) \breve{\gamma}_{\mu} \psi_{A}(x)$ does not depends on $x_{*}$ so $\int d x_{*} e^{-i \beta_{q} x_{*}}=2 \pi \delta\left(\beta_{q}\right)$.

Let us consider now the last two lines in the power expansion (4.6) of $\mathcal{J}_{A B}^{\mu}(x) \mathcal{J}_{B A \mu}(0)$ :

$$
\begin{aligned}
& \bar{\Psi}_{A}^{(1)}(x) \breve{\gamma}^{\mu} \Psi_{B}^{(0)}(x) \bar{\Psi}_{B}^{(0)}(0) \breve{\gamma}_{\mu} \Psi_{A}^{(0)}(0)+\bar{\Psi}_{A}^{(0)}(x) \breve{\gamma}^{\mu} \Psi_{B}^{(1)}(x) \bar{\Psi}_{B}^{(0)}(0) \breve{\gamma}_{\mu} \Psi_{A}^{(0)}(0) \\
& +\bar{\Psi}_{A}^{(0)}(x) \breve{\gamma}^{\mu} \Psi_{B}^{(0)}(x) \bar{\Psi}_{B}^{(1)}(0) \breve{\gamma}_{\mu} \Psi_{A}^{(0)}(0)+\bar{\Psi}_{A}^{(0)}(x) \breve{\gamma}^{\mu} \Psi_{B}^{(0)}(x) \bar{\Psi}_{B}^{(0)}(0) \breve{\gamma}_{\mu} \Psi_{A}^{(1)}(0)+\ldots
\end{aligned}
$$

After Fierz transformation (4.8) the first term in the above equation turns to

$$
\begin{aligned}
& \bar{\Psi}_{A}^{(0)}(x) \breve{\gamma}^{\mu} \Psi_{B}^{(0)}(x) \bar{\Psi}_{B}^{(0)}(0) \breve{\gamma}_{\mu} \Psi_{A}^{(1)}(0) \\
& =\frac{1+a^{2}}{2}\left\{\left[\bar{\Psi}_{A}^{m(0)}(x) \gamma_{\alpha} \Psi_{A}^{n(1)}(0)\right]\left[\bar{\Psi}_{B}^{n(0)}(0) \gamma^{\alpha} \Psi_{B}^{m(0)}(x)\right]+\left(\gamma_{\alpha} \otimes \gamma^{\alpha} \leftrightarrow \gamma_{\alpha} \gamma_{5} \otimes \gamma^{\alpha} \gamma_{5}\right)\right\} \\
& -a\left\{\left[\bar{\Psi}_{A}^{m(0)}(x) \gamma_{\alpha} \Psi_{A}^{n(1)}(0)\right]\left[\bar{\Psi}_{B}^{n(0)}(0) \gamma^{\alpha} \gamma_{5} \Psi_{B}^{m(0)}(x)\right]+\left(\gamma_{\alpha} \otimes \gamma^{\alpha} \gamma_{5} \leftrightarrow \gamma_{\alpha} \gamma_{5} \otimes \gamma^{\alpha}\right)\right\} \\
& +\left(1-a^{2}\right)\left\{\left[\bar{\Psi}_{A}^{m(0)}(x) \Psi_{A}^{n(1)}(0)\right]\left[\bar{\Psi}_{B}^{n(0)}(0) \Psi_{B}^{m(0)}(x)\right]-\left(1 \otimes 1 \leftrightarrow \gamma_{5} \otimes \gamma_{5}\right)\right\} \\
& =\frac{1+a^{2}}{2}\left\{\frac{2}{s}\left[\bar{\psi}_{A}^{m}(x) \not p_{2} \Psi_{1 A}^{n(1)}(0)\right]\left[\bar{\psi}_{B}^{n}(0) \not p_{1} \psi_{B}^{m}(x)\right]+\left(\not p_{2} \otimes \not p_{1} \leftrightarrow \not p_{2} \gamma_{5} \otimes \not p_{1} \gamma_{5}\right)\right\} \\
& -a\left\{\frac{2}{s}\left[\bar{\psi}_{A}^{m}(x) \not p_{2} \Psi_{1 A}^{n(1)}(0)\right]\left[\bar{\psi}_{B}^{n}(0) \not p_{1} \gamma_{5} \psi_{B}^{m}(x)\right]+\left(\not p_{2} \otimes \not p_{1} \gamma_{5} \leftrightarrow \not p_{2} \gamma_{5} \otimes \not p_{1}\right)\right\}+O\left(\frac{m_{\perp}^{8}}{s}\right) .
\end{aligned}
$$

\footnotetext{
${ }^{20}$ It is worth mentioning that if Z-boson is produced in the region of rapidity close to the projectile, the contribution (C.15) may be the most important since gluon parton densities at small $x_{B}$ are larger than quark ones.
} 
Since

$$
\not p_{2} \Psi_{1 A}^{(1)}=-\frac{g \not \not_{2} \not p_{1}}{s \beta} \gamma^{i} B_{i} \Psi_{A}+\frac{g \gamma^{i}}{s} \not p_{2} \frac{1}{\beta} \mathcal{P}_{i} \frac{1}{\alpha} \gamma^{j} B_{j} \Psi_{A}-\frac{2 g}{s} \not p_{2} \frac{1}{\beta}\left(\mathbb{A}_{\bullet}^{[1]}\right)^{(0)} \Psi_{A}
$$

we get

$$
\begin{gathered}
\bar{\Psi}_{A}^{(0)}(x) \breve{\gamma}^{\mu} \Psi_{B}^{(0)}(x) \bar{\Psi}_{B}^{(0)}(0) \breve{\gamma}_{\mu} \Psi_{A}^{(1)}(0) \\
=-\frac{1+a^{2}}{s^{2}} g\left\{\left[\bar{\psi}_{A}^{m}(x)\left(\not p_{2} \not p_{1} \gamma^{i}\left(\frac{1}{\beta} B_{i}\right)+\not p_{2} \gamma^{i} \gamma^{j} \frac{1}{\beta} \mathcal{P}_{i} B_{j} \frac{1}{\alpha}+2 \not p_{2} \frac{1}{\beta}\left(\mathbb{A}_{\bullet}^{[1]}\right)^{(0)}\right)^{n k} \psi_{A}^{k}(0)\right]\right. \\
\left.\times\left[\bar{\psi}_{B}^{n}(0) \not p_{1} \psi_{B}^{m}(x)\right]+\left(\not p_{2} \otimes \not p_{1} \leftrightarrow \not p_{2} \gamma_{5} \otimes \not p_{1} \gamma_{5}\right)\right\} \\
+\frac{2 a}{s^{2}} g\left\{\left[\bar{\psi}_{A}^{m}(x)\left(\not p_{2} \not p_{1} \gamma^{i}\left(\frac{1}{\beta} B_{i}\right)+\not p_{2} \gamma^{i} \gamma^{j} \frac{1}{\beta} \mathcal{P}_{i} B_{j} \frac{1}{\alpha}+2 \not p_{2} \frac{1}{\beta}\left(\mathbb{A}_{\bullet}^{[1]}\right)^{(0)}\right)^{n k} \psi_{A}^{k}(0)\right]\right. \\
\left.\times\left[\bar{\psi}_{B}^{n}(0) \not p_{1} \gamma_{5} \psi_{B}^{m}(x)\right]+\left(\not p_{2} \otimes \not p_{1} \gamma_{5} \leftrightarrow \not p_{2} \gamma_{5} \otimes \not p_{1}\right)\right\} .
\end{gathered}
$$

Let us start with the first term in parentheses in the second line of eq. (C.20). Using eq. (B.9) the corresponding matrix element can be rewritten as

$$
\begin{aligned}
& -\frac{1+a^{2}}{2 s N_{c}} g\left\langle A\left|\hat{\bar{\psi}}(x) \gamma^{i} \hat{\psi}(0)\right| A\right\rangle\left\langle B\left|\hat{\bar{\psi}}(0) \not p_{1}\left(\frac{1}{\beta}\left(\hat{A}_{i}+i \gamma_{5} \hat{\tilde{A}}_{i}\right)\right)(0) \hat{\psi}(x)\right| B\right\rangle \\
& -\frac{1+a^{2}}{2 s N_{c}} g\left\langle A\left|\hat{\bar{\psi}}(x) \gamma^{i} \gamma_{5} \hat{\psi}(0)\right| A\right\rangle\left\langle B\left|\hat{\bar{\psi}}(0) \not p_{1}\left(\frac{1}{\beta}\left(\gamma_{5} \hat{A}_{i}+i \hat{\tilde{A}}_{i}\right)\right)(0) \hat{\psi}(x)\right| B\right\rangle .
\end{aligned}
$$

Let us consider

$$
\begin{aligned}
& \int d x_{*} e^{-i \beta_{q} x_{*}}\left\langle B\left|\hat{\bar{\psi}}(0) \not p_{1}\left(\frac{1}{\beta}\left(\hat{A}_{i}+i \gamma_{5} \hat{\tilde{A}}_{i}\right)\right)(0) \hat{\psi}\left(x_{*}, x_{\perp}\right)\right| B\right\rangle \\
& =\frac{2 i}{s} \int d x_{*} e^{-i \beta_{q} x_{*}} \int_{-\infty}^{0} d x_{*}^{\prime} x_{*}^{\prime}\left\langle B\left|\hat{\bar{\psi}}(0) \not p_{1}\left[\hat{F}_{\bullet i}\left(x_{*}^{\prime}, 0_{\perp}\right)+i \gamma_{5} \hat{\tilde{F}}_{\bullet i}\left(x_{*}^{\prime}, 0_{\perp}\right)\right] \hat{\psi}\left(x_{*}, x_{\perp}\right)\right| B\right\rangle,
\end{aligned}
$$

where we used

$$
\frac{1}{\beta+i \epsilon} \hat{A}_{k}\left(z_{*}, z_{\perp}\right)=-i \int_{-\infty}^{z_{*}} d z_{*}^{\prime} \hat{A}_{k}\left(z_{*}^{\prime}, z_{\perp}\right)=-\frac{2 i}{s} \int_{-\infty}^{z_{*}} d z_{*}^{\prime}\left(z-z^{\prime}\right)_{*} \hat{F}_{\bullet k}\left(z_{*}^{\prime}, z_{\perp}\right) .
$$

Let us compare this matrix element to that of eq. (4.46):

$$
\begin{aligned}
& \int d x_{*} e^{-i \beta_{q} x_{*}}\left\langle B\left|\left(\hat{\bar{\psi}} \frac{1}{\beta-i \epsilon}\right)(0) \hat{A}_{i}(0) \Gamma \hat{\psi}\left(x_{*}, x_{\perp}\right)\right| B\right\rangle \\
& =-\frac{1}{\beta_{q}} \int d x_{*} e^{-i \beta_{q} x_{*}}\left\langle B\left|\left[\hat{\bar{\psi}}(0) \hat{A}_{i}(0)+\int_{-\infty}^{0} d x_{*}^{\prime} \hat{\bar{\psi}}\left(x_{*}^{\prime}, 0_{\perp}\right) \frac{2}{s} \hat{F}_{\bullet}(0)\right] \Gamma \hat{\psi}\left(x_{*}, x_{\perp}\right)\right| B\right\rangle .
\end{aligned}
$$

We see that $\frac{1}{\beta_{z}}$ in eq. (C.23) is traded for an extra $x_{*}^{\prime} \sim 1$ in eq. (C.22) (recall that $x_{*}^{\prime}$ in the target matrix elements is inversely proportional to characteristic $\beta$ 's in the target which are of order 1). Consequently, power correction due to matrix element (C.22) can be neglected 
in our kinematic region since the matrix element (C.21) is $\sim \frac{m_{\perp}^{2}}{s}$ rather than $\sim \frac{m_{\perp}^{2}}{\alpha_{q} \beta_{q} s}$. Similarly, the second term in eq. (C.21) does not contribute in our kinematical region.

This is the same reason why we neglected power corrections (C.6). In general, as we discussed in ref. [12], the way to figure out integrations that give $\frac{1}{\beta_{q}}$ is very simple: take $\beta_{q} \rightarrow 0$ and check if there is an infinite integration of the type $\int_{-\infty}^{x_{*}} d x_{*}^{\prime}$ without any integrand. Similarly, the factor $\frac{1}{\alpha_{q}}$ can be figured out from (possible) unrestricted integrals over $x_{\bullet}^{\prime}$ after one sets $\alpha_{q}=0$ in the relevant matrix element. Note that to get the terms $\sim \frac{1}{Q^{2}}=\frac{1}{\alpha_{q} \beta_{q} s}$ we need to find contributions which satisfy both of the above conditions.

Next, consider the second term in parentheses in the second line of eq. (C.20). Using eq. (4.44) the corresponding matrix element can be rewritten as

$$
\begin{aligned}
- & \frac{1+a^{2}}{s^{2}} g\left[\bar{\psi}_{A}^{m}(x) \not p_{2} \gamma^{i} \gamma^{j}\left(\frac{1}{\beta} \mathcal{P}_{i} B_{j}\right)^{n k} \frac{1}{\alpha} \psi_{A}^{k}(0)\right]\left[\bar{\psi}_{B}^{n}(0) \not p_{1} \psi_{B}^{m}(x)\right] \\
= & -\frac{1+a^{2}}{s^{2}} g\left[\bar{\psi}_{A}^{m}(x) \not p_{2} \gamma^{i} \gamma^{j}\left(\frac{1}{\beta} B_{j}\right)^{n k} \frac{1}{\alpha} i \partial_{i} \psi_{A}^{k}(0)+g \bar{\psi}_{A}^{m}(x) \not p_{2} \gamma^{i} \gamma^{j}\left(A_{i} \frac{1}{\beta} B_{j}\right)^{n k} \frac{1}{\alpha} \psi_{A}^{k}(0)\right. \\
& \left.+\bar{\psi}_{A}^{m}(x) \not p_{2} \gamma^{i} \gamma^{j} \frac{1}{\beta}\left(i \partial_{i} B_{j}+g B_{i} B_{j}\right)^{n k} \frac{1}{\alpha} \psi_{A}^{k}(0)\right]\left[\bar{\psi}_{B}^{n}(0) \not p_{1} \psi_{B}^{m}(x)\right] \\
\stackrel{\text { f.int. }}{\rightarrow}-\frac{1+a^{2}}{N_{c} s^{2}} g\left\langle A\left|\hat{\bar{\psi}}(x) \not p_{2} \gamma^{i} \gamma^{j} \frac{1}{\alpha} i \partial_{i} \hat{\psi}(0)\right| A\right\rangle\left\langle B\left|\hat{\bar{\psi}}(0) \not p_{1}\left(\frac{1}{\beta} \hat{A}_{j}\right)(0) \hat{\psi}(x)\right| B\right\rangle & \\
& +\frac{1+a^{2}}{N_{c}\left(N_{c}^{2}-1\right) s^{2}} g^{2}\left\langle A\left|\hat{\bar{\psi}}(x) \not p_{2} \gamma^{i} \gamma^{j} \hat{A}_{i} \frac{1}{\alpha} \hat{\psi}(0)\right| A\right\rangle\left\langle B\left|\hat{\bar{\psi}}(0) \not p_{1}\left[\frac{1}{\beta} \hat{A}_{j}(0)\right] \hat{\psi}(x)\right| B\right\rangle \\
& -\frac{1+a^{2}}{N_{c} s^{2}} g\left\langle A\left|\hat{\bar{\psi}}(x) \not p_{2} \gamma^{i} \gamma^{j} \frac{1}{\alpha} \psi_{A}(0)\right| A\right\rangle\left\langle B\left|\hat{\bar{\psi}}(0) \not p_{1}\left[\frac{1}{\beta}\left(i \partial_{i} \hat{A}_{j}+g \hat{A}_{i} \hat{A}_{j}\right)(0)\right] \hat{\psi}(x)\right| B\right\rangle . \quad(\mathrm{C} .24
\end{aligned}
$$

Using eq. (B.9) we get

$$
\begin{aligned}
- & \frac{1+a^{2}}{s^{2}} g\left[\bar{\psi}_{a}^{m}(x) \not p_{2} \gamma^{i} \gamma^{j}\left(\frac{1}{\beta} \mathcal{P}_{i} B_{j}\right)^{n k} \frac{1}{\alpha} \psi_{a}^{k}(0)\right]\left[\bar{\psi}_{b}^{n}(0) \not p_{1} \psi_{b}^{m}(x)\right]+\left(\not p_{2} \otimes \not p_{1} \leftrightarrow p_{2} \gamma_{5} \otimes \not p_{1} \gamma_{5}\right) \\
\stackrel{\text { f.int. }}{\rightarrow}- & \frac{1+a^{2}}{N_{c} s^{2}} g\left\langle A\left|\hat{\bar{\psi}}(x) \not p_{2} \frac{1}{\alpha} i \partial_{i} \hat{\psi}(0)\right| A\right\rangle\left\langle B\left|\hat{\bar{\psi}}(0) \not p_{1}\left(\frac{1}{\beta}\left(\hat{A}^{i}-i \hat{\tilde{A}}^{i} \gamma_{5}\right)(0)\right) \hat{\psi}(x)\right| B\right\rangle \\
& +\frac{1+a^{2}}{N_{c}\left(N_{c}^{2}-1\right) s^{2}} g^{2}\left\langle A\left|\hat{\bar{\psi}}(x) \not p_{2} \hat{A}_{i} \frac{1}{\alpha} \hat{\psi}(0)\right| A\right\rangle\left\langle B\left|\hat{\bar{\psi}}(0) \not p_{1}\left(\frac{1}{\beta}\left(\hat{A}^{i}-i \hat{\tilde{A}}^{i} \gamma_{5}\right)(0)\right) \hat{\psi}(x)\right| B\right\rangle \\
& -\frac{1+a^{2}}{N_{c} s^{2}} g\left\langle A\left|\hat{\bar{\psi}}(x) \not p_{2} \frac{1}{\alpha} \psi_{A}(0)\right| A\right\rangle\left\langle B\left|\hat{\bar{\psi}}(0) \not p_{1}\left(\frac{1}{\beta}\left(i \partial_{i} \hat{A}^{i}+g \hat{A}_{i} \hat{A}^{i}\right)(0)\right) \hat{\psi}(x)\right| B\right\rangle \\
& -\frac{2\left(1+a^{2}\right)}{N_{c} s^{3}} g\left\langle A\left|\hat{\bar{\psi}}(x) \not p_{2} \frac{1}{\alpha} \psi_{A}(0)\right| A\right\rangle\left\langle B\left|\hat{\bar{\psi}}(0) \not p_{1} \gamma_{5}\left(\frac{1}{\beta} \hat{\tilde{F}}_{* \bullet}(0)\right) \hat{\psi}(x)\right| B\right\rangle \\
& +\left(\not p_{2} \otimes \not p_{1} \leftrightarrow \not p_{2} \gamma_{5} \otimes \not p_{1} \gamma_{5}\right) .
\end{aligned}
$$

It is easy to see that projectile matrix elements lead to terms $\sim \frac{1}{\alpha_{q}}$ after integration over $x_{\bullet}$, for example

$$
\begin{aligned}
& \int d x_{\bullet} e^{-i \alpha_{q} x \bullet}\left\langle A\left|\hat{\bar{\psi}}(x) p_{2} \hat{A}_{i}(0) \frac{1}{\alpha} \hat{\psi}(0)\right| A\right\rangle=\frac{2}{\alpha_{q} s} \int d x_{\bullet} e^{i \alpha_{q} x_{\bullet}} \int_{-\infty}^{x_{\bullet}} d x_{\bullet}^{\prime} \\
& \quad \times\langle A| \hat{\bar{\psi}}(0) \not p_{2}\left[\hat{F}_{* i}\left(x_{\bullet},-x_{\perp}\right) \hat{\psi}\left(x_{\bullet}^{\prime},-x_{\perp}\right)+\hat{F}_{* i}\left(x_{\bullet}^{\prime},-x_{\perp}\right) \hat{\psi}\left(x_{\bullet},-x_{\perp}\right)|A\rangle .\right.
\end{aligned}
$$


On the other hand, the target matrix elements cannot give a $\frac{1}{\beta_{q}}$ factor. For the first two lines in the r.h.s. of eq. (C.25) we proved this in eq. (C.22) above. As to the last lines in eq. (C.25), the target matrix element can be rewritten as

$$
\begin{aligned}
& \int d x_{*} e^{-i \beta_{q} x_{*}}\left\langle B\left|\hat{\bar{\psi}}(0) \not p_{1}\left(\frac{1}{\beta}\left(i \partial_{i} \hat{A}^{i}+g \hat{A}_{i} \hat{A}^{i}\right)(0)\right) \hat{\psi}\left(x_{*}, x_{\perp}\right)\right| B\right\rangle=-\frac{2 i}{s} \int d x_{*} e^{-i \beta_{q} x_{*}} \int_{-\infty}^{0} d x_{*}^{\prime} \\
& \times\left\langle B\left|\hat{\bar{\psi}}(0) \not p_{1}\left(\int_{-\infty}^{x_{*}^{\prime}} d x_{*}^{\prime \prime}\left[i \hat{D}^{i} \hat{F}_{\bullet}\left(x_{*}^{\prime \prime}, 0_{\perp}\right)+\frac{2 g}{s} \hat{F}_{\bullet}{ }^{i}\left(x_{*}^{\prime \prime}, 0_{\perp}\right) \int_{-\infty}^{x_{*}^{\prime \prime}} d x_{*}^{\prime \prime \prime} \hat{F}_{\bullet i}\left(x_{*}^{\prime \prime \prime}, 0_{\perp}\right)\right]\right) \hat{\psi}\left(x_{*}, x_{\perp}\right)\right| B\right\rangle
\end{aligned}
$$

and

$$
\begin{aligned}
\int d x_{*} & e^{-i \beta_{q} x_{*}}\left\langle B\left|\hat{\bar{\psi}}(0) \not p_{1} \gamma_{5}\left(\frac{1}{\beta} \hat{\tilde{F}}_{* \bullet}(0)\right) \hat{\psi}\left(x_{*}, x_{\perp}\right)\right| B\right\rangle \\
= & -i \int d x_{*} e^{-i \beta_{q} x_{*}} \int_{-\infty}^{0} d x_{*}^{\prime}\left\langle B\left|\hat{\bar{\psi}}(0) \not p_{1} \gamma_{5} \hat{\tilde{F}}_{* \bullet}\left(x_{*}^{\prime}, 0_{\perp}\right) \hat{\psi}\left(x_{*}, x_{\perp}\right)\right| B\right\rangle .
\end{aligned}
$$

We see that at $\beta_{q}=0$ there are no unrestricted integration over any longitudinal variable so the r.h.s. of these equations cannot give $\frac{1}{\beta_{q}}$ factor and therefore the contribution to $W(q)$ is $\sim \frac{m_{\perp}^{2}}{\alpha_{q} s} \ll \frac{m_{\perp}^{2}}{Q^{2}}$.

Finally, we should consider the third term in eq. (C.20)

$$
\begin{aligned}
- & 2 \frac{1+a^{2}}{s^{2}} g\left[\bar{\psi}_{A}^{m}(x) \not p_{2} \frac{1}{\beta}\left(\mathbb{A}_{\bullet}^{[1] n l}\right)^{(0)} \psi_{A}^{l}(0)\right]\left[\bar{\psi}_{B}^{n}(0) \not p_{1} \psi_{B}^{m}(x)\right] \\
= & -\frac{1+a^{2}}{s^{2}} g^{2}\left[\bar{\psi}_{A}^{m}(x) \not p_{2}\left(\frac{1}{\alpha} A_{j}(0)\right)^{n k} \psi_{A}^{l}(0)\right]\left[\bar{\psi}_{B}^{n}(0) \not p_{1}\left(\frac{1}{\beta} B^{j}(0)\right)^{k l} \psi_{B}^{m}(x)\right] \\
& +\frac{1+a^{2}}{s^{2}} g^{2}\left[\bar{\psi}_{A}^{m}(x) \not p_{2}\left(\frac{1}{\alpha} A_{j}(0)\right)^{k l} \psi_{A}^{l}(0)\right]\left[\bar{\psi}_{B}^{n}(0) \not p_{1}\left(\frac{1}{\beta} B^{j}(0)\right)^{n k} \psi_{B}^{m}(x)\right],
\end{aligned}
$$

where we used eq. (A.5) for $\left(\mathbb{A}_{\bullet}^{[1]}\right)^{(0)}$. Taking projectile and target matrix elements and separating color-singlet contributions using eq. (4.44), we obtain

$$
-\frac{\left(1+a^{2}\right) N_{c}}{s^{2}\left(N_{c}^{2}-1\right)} g^{2}\left\langle A\left|\hat{\bar{\psi}}(x) \not p_{2}\left(\frac{1}{\alpha} \hat{A}_{j}(0)\right) \hat{\psi}(0)\right| A\right\rangle\left\langle B\left|\hat{\bar{\psi}}(0) \not p_{1}\left(\frac{1}{\beta} \hat{A}^{j}(0)\right) \hat{\psi}(x)\right| B\right\rangle
$$

It has been demonstrated in eq. (C.22) that such matrix elements cannot give $\frac{1}{\alpha_{q}}$ and $\frac{1}{\beta_{q}}$ so their contribution to $W(q)$ is small in our kinematical region. Moreover, since the above arguments do not depend on presence (or absence) of $\gamma_{5}$, we proved that all terms in eq. (C.20) give small contributions at $\alpha_{q}, \beta_{q} \ll 1$. In a similar way, one can demonstrate that the other three terms in eq. (C.17) can be neglected. 
Open Access. This article is distributed under the terms of the Creative Commons Attribution License (CC-BY 4.0), which permits any use, distribution and reproduction in any medium, provided the original author(s) and source are credited.

\section{References}

[1] J. Collins, Foundations of perturbative QCD, Cambridge University Press (2013).

[2] J.C. Collins and D.E. Soper, Parton Distribution and Decay Functions, Nucl. Phys. B 194 (1982) 445 [INSPIRE].

[3] J.C. Collins, D.E. Soper and G.F. Sterman, Transverse Momentum Distribution in Drell-Yan Pair and $W$ and $Z$ Boson Production, Nucl. Phys. B 250 (1985) 199 [INSPIRE].

[4] X.-d. Ji, J.-p. Ma and F. Yuan, QCD factorization for semi-inclusive deep-inelastic scattering at low transverse momentum, Phys. Rev. D 71 (2005) 034005 [hep-ph/0404183] [INSPIRE].

[5] M.G. Echevarria, A. Idilbi and I. Scimemi, Factorization Theorem For Drell-Yan At Low $q_{T}$ And Transverse Momentum Distributions On-The-Light-Cone, JHEP 07 (2012) 002 [arXiv:1111.4996] [INSPIRE].

[6] C.W. Bauer, S. Fleming, D. Pirjol and I.W. Stewart, An Effective field theory for collinear and soft gluons: Heavy to light decays, Phys. Rev. D 63 (2001) 114020 [hep-ph/0011336] [INSPIRE].

[7] C.W. Bauer, D. Pirjol and I.W. Stewart, Soft collinear factorization in effective field theory, Phys. Rev. D 65 (2002) 054022 [hep-ph/0109045] [INSPIRE].

[8] C.W. Bauer, S. Fleming, D. Pirjol, I.Z. Rothstein and I.W. Stewart, Hard scattering factorization from effective field theory, Phys. Rev. D 66 (2002) 014017 [hep-ph/0202088] [INSPIRE].

[9] T. Becher and M. Neubert, Drell-Yan Production at Small $q_{T}$, Transverse Parton Distributions and the Collinear Anomaly, Eur. Phys. J. C 71 (2011) 1665 [arXiv: 1007.4005] [INSPIRE].

[10] J.-Y. Chiu, A. Jain, D. Neill and I.Z. Rothstein, A Formalism for the Systematic Treatment of Rapidity Logarithms in Quantum Field Theory, JHEP 05 (2012) 084 [arXiv:1202.0814] [INSPIRE].

[11] J. Collins and T. Rogers, Understanding the large-distance behavior of transverse-momentum-dependent parton densities and the Collins-Soper evolution kernel, Phys. Rev. D 91 (2015) 074020 [arXiv:1412.3820] [INSPIRE].

[12] I. Balitsky and A. Tarasov, Higher-twist corrections to gluon TMD factorization, JHEP 07 (2017) 095 [arXiv: 1706.01415] [INSPIRE].

[13] F. Landry, R. Brock, G. Ladinsky and C.P. Yuan, New fits for the nonperturbative parameters in the CSS resummation formalism, Phys. Rev. D 63 (2001) 013004 [hep-ph/9905391] [INSPIRE].

[14] J.-w. Qiu and X.-f. Zhang, Role of the nonperturbative input in QCD resummed Drell-Yan $Q_{T}$ distributions, Phys. Rev. D 63 (2001) 114011 [hep-ph/0012348] [INSPIRE].

[15] F. Landry, R. Brock, P.M. Nadolsky and C.P. Yuan, Tevatron Run-1 Z boson data and Collins-Soper-Sterman resummation formalism, Phys. Rev. D 67 (2003) 073016 [hep-ph/0212159] [INSPIRE]. 
[16] S. Mantry and F. Petriello, Transverse Momentum Distributions in the Non-Perturbative Region, Phys. Rev. D 84 (2011) 014030 [arXiv:1011.0757] [INSPIRE].

[17] P. Sun, J. Isaacson, C.P. Yuan and F. Yuan, Universal Non-perturbative Functions for SIDIS and Drell-Yan Processes, Int. J. Mod. Phys. A 33 (2018) 1841006 [arXiv:1406.3073] [INSPIRE].

[18] U. D'Alesio, M.G. Echevarria, S. Melis and I. Scimemi, Non-perturbative QCD effects in $q_{T}$ spectra of Drell-Yan and Z-boson production, JHEP 11 (2014) 098 [arXiv:1407.3311] [INSPIRE].

[19] S. Catani, D. de Florian, G. Ferrera and M. Grazzini, Vector boson production at hadron colliders: transverse-momentum resummation and leptonic decay, JHEP 12 (2015) 047 [arXiv:1507.06937] [INSPIRE].

[20] A. Bacchetta, F. Delcarro, C. Pisano, M. Radici and A. Signori, Extraction of partonic transverse momentum distributions from semi-inclusive deep-inelastic scattering, Drell-Yan and Z-boson production, JHEP 06 (2017) 081 [arXiv:1703.10157] [INSPIRE].

[21] I. Scimemi and A. Vladimirov, Analysis of vector boson production within TMD factorization, Eur. Phys. J. C 78 (2018) 89 [arXiv:1706.01473] [InSPIRE].

[22] P.J. Mulders and R.D. Tangerman, The Complete tree level result up to order $1 / Q$ for polarized deep inelastic leptoproduction, Nucl. Phys. B 461 (1996) 197 [Erratum ibid. B 484 (1997) 538] [hep-ph/9510301] [INSPIRE].

[23] Particle Data Group collaboration, C. Patrignani et al., Review of Particle Physics, Chin. Phys. C 40 (2016) 100001 [inSPIRE].

[24] I.I. Balitsky and V.M. Braun, Nonlocal Operator Expansion for Structure Functions of $e^{+} e^{-}$ Annihilation, Phys. Lett. B 222 (1989) 123 [INSPIRE].

[25] I.I. Balitsky and V.M. Braun, The Nonlocal operator expansion for inclusive particle production in $e^{+} e^{-}$annihilation, Nucl. Phys. B 361 (1991) 93 [INSPIRE].

[26] I.I. Balitsky and V.M. Braun, Valleys in Minkowski space and instanton induced cross-sections, Nucl. Phys. B 380 (1992) 51 [inSPIRE].

[27] F. Gelis and R. Venugopalan, Large mass q $\bar{q}$ production from the color glass condensate, Phys. Rev. D 69 (2004) 014019 [hep-ph/0310090] [InSPIRE].

[28] F. Gelis and R. Venugopalan, Particle production in field theories coupled to strong external sources, Nucl. Phys. A 776 (2006) 135 [hep-ph/0601209] [InSPIRE].

[29] F. Gelis, T. Lappi and R. Venugopalan, High energy scattering in Quantum Chromodynamics, Int. J. Mod. Phys. E 16 (2007) 2595 [arXiv:0708.0047] [InSPIRE].

[30] A.V. Belitsky, X. Ji and F. Yuan, Final state interactions and gauge invariant parton distributions, Nucl. Phys. B 656 (2003) 165 [hep-ph/0208038] [INSPIRE].

[31] I.Z. Rothstein and I.W. Stewart, An Effective Field Theory for Forward Scattering and Factorization Violation, JHEP 08 (2016) 025 [arXiv: 1601.04695] [INSPIRE].

[32] R.D. Tangerman and P.J. Mulders, Intrinsic transverse momentum and the polarized Drell-Yan process, Phys. Rev. D 51 (1995) 3357 [hep-ph/9403227] [INSPIRE].

[33] Y.V. Kovchegov and M.D. Sievert, Calculating TMDs of a Large Nucleus: Quasi-Classical Approximation and Quantum Evolution, Nucl. Phys. B 903 (2016) 164 [arXiv:1505.01176] [INSPIRE]. 
[34] J. Zhou, F. Yuan and Z.-T. Liang, Hyperon Polarization in Unpolarized Scattering Processes, Phys. Rev. D 78 (2008) 114008 [arXiv:0808.3629] [InSPIRE].

[35] A. Bacchetta, M. Boglione, A. Henneman and P.J. Mulders, Bounds on transverse momentum dependent distribution and fragmentation functions, Phys. Rev. Lett. 85 (2000) 712 [hep-ph/9912490] [INSPIRE].

[36] I. Balitsky and G.A. Chirilli, Next-to-leading order evolution of color dipoles, Phys. Rev. D 77 (2008) 014019 [arXiv: 0710.4330] [INSPIRE].

[37] I. Balitsky and A. Tarasov, Rapidity evolution of gluon TMD from low to moderate X, JHEP 10 (2015) 017 [arXiv: 1505.02151] [INSPIRE].

[38] I. Balitsky and A. Tarasov, Gluon TMD in particle production from low to moderate $x$, JHEP 06 (2016) 164 [arXiv: 1603.06548] [INSPIRE].

[39] I. Moult, I.W. Stewart and G. Vita, A subleading operator basis and matching for $g g \rightarrow H$, JHEP 07 (2017) 067 [arXiv: 1703.03408] [INSPIRE].

[40] R. Goerke and M. Luke, Power Counting and Modes in SCET, JHEP 02 (2018) 147 [arXiv:1711.09136] [INSPIRE].

[41] M. Beneke, M. Garny, R. Szafron and J. Wang, Anomalous dimension of subleading-power $N$-jet operators, JHEP 03 (2018) 001 [arXiv: 1712.04416] [INSPIRE].

[42] S.J. Brodsky, D.S. Hwang and I. Schmidt, Final state interactions and single spin asymmetries in semiinclusive deep inelastic scattering, Phys. Lett. B 530 (2002) 99 [hep-ph/0201296] [INSPIRE].

[43] J.C. Collins, Leading twist single transverse-spin asymmetries: Drell-Yan and deep inelastic scattering, Phys. Lett. B 536 (2002) 43 [hep-ph/0204004] [INSPIRE].

[44] I. Balitsky, Operator expansion for high-energy scattering, Nucl. Phys. B 463 (1996) 99 [hep-ph/9509348] [INSPIRE].

[45] I. Balitsky, High-energy QCD and Wilson lines, hep-ph/0101042 [INSPIRE]. 